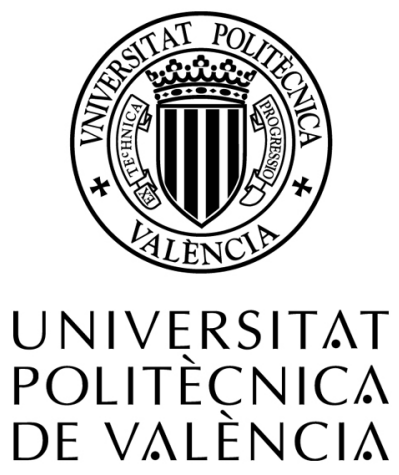

Universitat Politècnica de València - Departamento de Matemática Aplicada

Doctorado en Matemáticas

Tesis de Doctorado

\title{
"Desarrollo de modelos matemáticos y análisis de sensibilidad para el estudio energético de edificaciones"
}

Roberto Robledo Fava

\author{
Directores: \\ Arturo Alberto Castillo Guzmán (UANL) \\ Pedro Fernández de Córdoba Castellá (UPV) \\ María Sonia Zaragoza Fernández (UDC)
}

Diciembre de 2018 



\section{Resumen}

Presentamos un análisis de la sensibilidad de los resultados de simulaciones numéricas basadas en Building Information Modeling (BIM), a las variaciones en los valores de los parámetros humanos subjetivos (SHP) definidos en la norma ISO 7730 , como vestimenta o actividad. Nuestro análisis muestra que los pequeños cambios en los SHP pueden producir oscilaciones significativas en los resultados de los cálculos numéricos, que, en nuestro caso, se realizaron con el software TRNSYS. Para verificar la validez de nuestro enfoque, hemos implementado un código Monte Carlo, para analizar los efectos principales de las diferentes variables de forma sistemática. 


\section{Abstract}

We present an analysis of the sensitivity of the results of Building Information Modeling (BIM)-based numerical simulations, to variations in the values of subjective human parameters (SHPs) defined in the ISO standard 7730, like clothing or activity. Our analysis shows that slight changes on SHPs may yield significant oscillations on the results of the numerical calculations, which in our case were made with TRNSYS software. To check the validity of our approach, we have implemented a Monte Carlo code, to analyze the main effects of the different variables in a systematic way. 


\section{Resum}

Presentem una anàlisi de la sensibilitat dels resultats de simulacions numèriques basades en Building Information Modeling (BIM), a les variacions en els valors dels paràmetres humans subjectius (SHP) definits en la norma ISO 7730, com a vestimenta o activitat. La nostra anàlisi mostra que els xicotets canvis en els SHP poden produir oscil-lacions significatives en els resultats dels càlculs numèrics, que, en el nostre cas, es van realitzar amb el programari TRNSYS. Per a verificar la validesa del nostre enfocament, hem implementat un codi Monte Carlo, per a analitzar els efectes principals de les diferents variables de forma sistemàtica. 



\section{Agradecimientos Institucionales}

En primer lugar, quiero agradecer al Consejo Nacional de Ciencia y Tecnología, CONACYT, y de manera muy especial al Instituto de Innovación y Transferencia de Tecnología del Estado de Nuevo León, i2t2, quienes en conjunto me brindaron el apoyo económico para realizar mis estudios de Doctorado en el Extranjero con el número de Beca: 298503 y CVU: 559309.

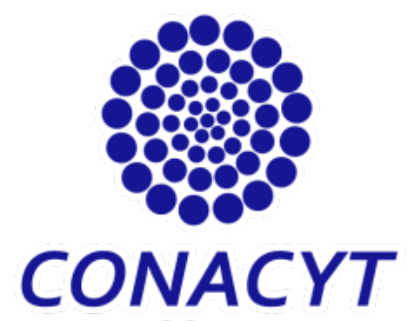

Consejo Nacional de Ciencia y Tecnología

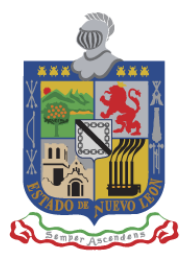

Instituto

de Innovación

y Transferencia

de Tecnología

Nuevoleón

GOBIERNO DEL ESTADO

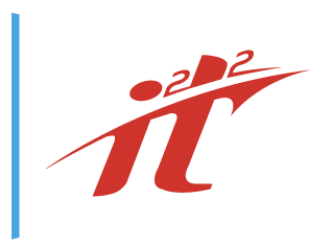


El éxito de este trabajo de tesis doctoral fue posible gracias a la colaboración y participación de las diferentes instituciones educativas, a las que expreso mi más sincero agradecimiento por haber puesto a mi disposición todos los recursos y medios necesarios para la realización de mis estudios doctorales, reiterando el firme deseo de contar con su colaboración para trabajos futuros.

A la Universidad Politécnica de Valencia, UPV.

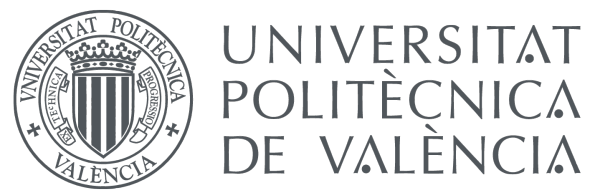

A la Universidade Da Coruña, UDC.

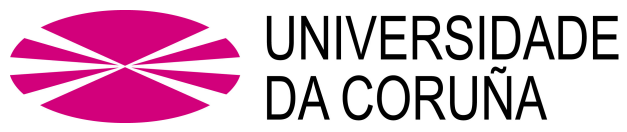

A la Universidad Autónoma de Nuevo León, UANL.

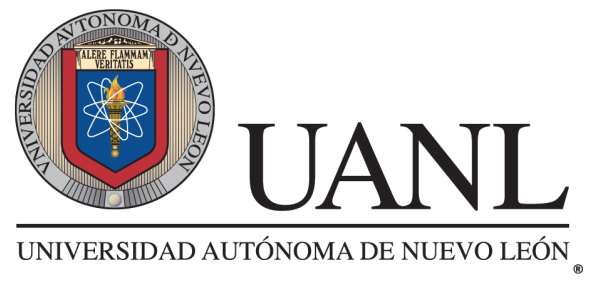

A la Facultad de Ciencias Físico-Matemáticas, FCFM.

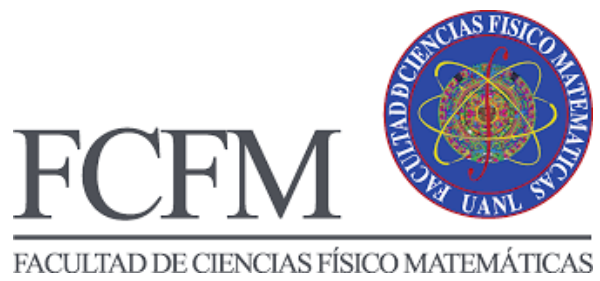




\section{Agradecimientos Personales}

Esta tesis doctoral, si bien ha requerido de esfuerzo y mucha dedicación por parte del autor y de sus directores de tesis, no hubiese sido posible su finalización sin la cooperación desinteresada de todas y cada una de las personas que a continuación citaré y muchas de las cuales han sido un soporte esencial para culminar mis estudios de doctorado.

Le agradezco a Dios por haberme acompañado y guiado a lo largo de esta etapa de doctorado, por ser mi fortaleza en mis momentos de debilidad, por brindarme una vida llena de aprendizajes y por haber puesto en mi camino a aquellas personas que han sido mi soporte y compañía durante todo el periodo de estudio.

En primer lugar, quiero agradecer a mi amada compañera de vida, mi esposa y amiga, Mónica Cynthia Hernández Luna. Mónica, gracias por acompañarme en este proceso, por ser el pilar que me mantuvo de pie aun estando ambos en el extranjero; tu amor, comprensión, paciencia y fortaleza permitieron alcanzar esta dichosa victoria en la vida, de la cual formas parte, el poder haber culminado esta tesis con éxito. Soy dichoso de poder ser agradecido contigo, que siempre te preocupaste por mí a cada momento y que continuamente quisiste lo mejor para mi porvenir. Sé que no ha sido un proceso fácil y que me ayudaste hasta donde te era posible, incluso más que eso. Ha sido maravilloso trabajar a tu lado, eres una mujer estupenda, inteligente y de la cual tenemos mucho que aprender, sin ti este trabajo no sería posible. En estas líneas es difícil explicar lo agradecido que estoy contigo, por cada una de las cosas que has hecho por mí, pero ten seguro que haré hasta lo imposible por demostrarte en esta nueva etapa de nuestra vida, cuanto te amo y lo importante que eres para mí. Busco rendir mi ser y volar contigo, quisiera compartir toda mi vida contigo (Carla Morrison).

Me gustaría agradecer de manera especial y sincera a mis directores de tesis, Dr. Pedro Fernández de Córdoba Castellá, Dra. Sonia Zaragoza Fernández y Dr. Arturo Castillo Guzmán por aceptarme para realizar esta tesis doctoral bajo su dirección. Su apoyo y confianza en mi trabajo y su capacidad para guiar mis ideas ha sido un aporte invaluable, no solamente en el desarrollo de esta tesis, sino también en mi formación como investigador. Las ideas propias, siempre enmarcadas en su orientación y rigurosidad, han sido la clave del excelente trabajo que hemos realizado juntos, el cual no se puede concebir sin su siempre oportuna participación. Les agradezco también el haberme facilitado siempre los medios suficientes para llevar a cabo todas las actividades propuestas durante el desarrollo de esta tesis. 
Estaré siempre eternamente agradecido a mi familia en Coahuila, México, mis padres Roberto Robledo Mendoza y Olga Alicia Fava Cortez, que desde el primer momento me brindan todo el apoyo, colaboración y cariño sin ningún interés, siendo ellos mi principal motor, quienes, sin duda me han motivado y alentado a seguir adelante a pesar de estar lejos físicamente. Agradezco esas horas que pasábamos hablando desde un sábado hasta un domingo, sin importar la diferencia de horario de 7 horas, en las cuales nos encantaba escuchar lo bueno y malo que nos había pasado durante la semana, sé que no ha habido día que no estuviéramos en sus bendiciones. Hermana Cinthia, te agradezco por siempre estar al pendiente de mis padres y de mí; sé que durante el último año hemos pasado momentos difíciles, y esto ha permitido estar más unido a ti y a tu familia: Humberto, Romina y Sofía. Agradecido estoy con mi hermana Nayeli, por apoyarme y hacerme sentir como en casa cada que estaba de estancia en Nuevo León, que, junto con Erick, siempre me han procurado. A mi familia en Salamanca, México, mis suegros Ángeles Luna y Jesús Hernández, cuñado Ángel, que siempre han estado pendientes de mí y apoyándome a cada momento. Porque a pesar de la distancia, han sido todos ustedes, quienes me brindan la fortaleza, apoyo y alegría necesaria para seguir adelante.

Un agradecimiento especial al Dr. Arturo Castillo que en todo momento ha estado a mi lado, no solo para aconsejarme si no para brindarme una sincera amistad, sigo convencido que una de mis mejores decisiones profesionales fue llegar al laboratorio a trabajar contigo, quizás las palabras no bastan para agradecerte el que hayas confiado en mí para los proyectos que hemos desarrollado.

Mi más sincera gratitud al Dr. Romeo Selvas, por haber confiado en mí para sus proyectos, el cual hoy llega a su fin y marca un inicio a una nueva etapa de colaboración. Además, de la parte académica, agradezco su apoyo incondicional en cada una de mis decisiones personales, así como su amistad y consejos.

Sin pasar por alto a mis amigos Diego Alarcón y Daniel Fuli, a quienes quiero agradecerles por siempre abrirme las puertas de su casa y formar una familia, nunca olvidaré el detalle de acompañarme en uno de los acontecimientos más importantes de mi vida, sin duda fueron piezas clave para mi entera felicidad. Porque en su compañía las cosas malas se convierten en buenas, la tristeza se transforma en alegría y la soledad no existe.

Quisiera dejar por escrito mi agradecimiento profundo a tres personas muy especiales que quiero con toda el alma, y que me han mostrado una infinidad de veces que siempre formamos parte de su familia: Paula, Ramón y Chloe.

Por ultimo quisiera agradecer a cada uno de mis familiares y amigos que a pesar de la distancia me han mostrado el gran cariño que me tienen, en especial Ana Hernández López, Roberto Álvarez y Valentín Guzmán. 


\section{Índice general}

Lista de figuras

XIII

Lista de tablas

XV

1. Introducción $\quad \mathbf{1}$

1.1. Introducción . . . . . . . . . . . . . . . . 1

1.2. Hipótesis . . . . . . . . . . . . . . . . . . . . 1

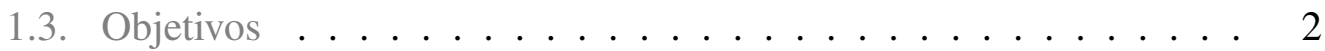

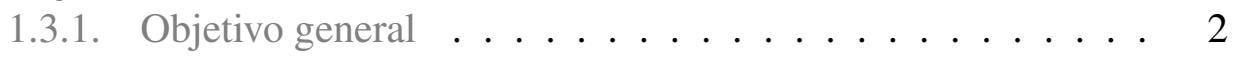

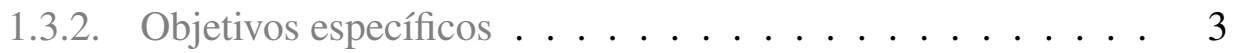

1.4. Motivación .................... 3

1.5. Estructura de la tesis ........................ 4

1.6. Metodología de la tesis ............... 5

2. Marco teórico $\quad 7$

2.1. Introducción .................... 7

2.2. Consumo energético del sector de los edificios . . . . . . . . . 8

2.3. Niveles de ambiente interior .............. . . . 8

2.3.1. Calidad del aire . . . . . . . . . . . . . . 9

2.3.2. Confort visual . . . . . . . . . . . . . . . . 10

2.3.3. Confort acústico . . . . . . . . . . . . . . . . 11

2.3.4. Confort térmico . . . . . . . . . . . . . . . . . . . 12

2.4. Índices PMV y PPD . . . . . . . . . . . . . . . . . . . . . . . 14

2.5. Simulación energética de edificios . . . . . . . . . . . . . 16

2.5.1. Energy Plus $\mathbb{R} \ldots \ldots \ldots \ldots \ldots$

2.5.2. ESP-r $\ldots \ldots \ldots \ldots \ldots \ldots \ldots$

2.5.3. TRNSYS $\mathrm{R} \ldots \ldots \ldots \ldots \ldots \ldots \ldots$

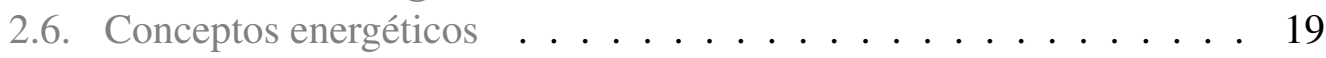

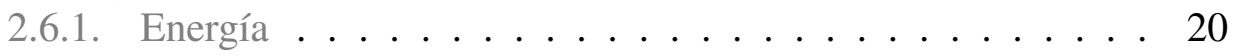

2.6.2. Demanda de energía . . . . . . . . . . . . . . . 21

2.6.3. Rendimiento energético .............. 22 
2.6.4. Rendimiento térmico . . . . . . . . . . . . . . 22

2.6.5. Consumo de energía . . . . . . . . . . . . . . . 22

2.7. Análisis de sensibilidad . . . . . . . . . . . . . . . . 23

2.8. Aplicación de herramientas de simulación y análisis de sensibilidad 23

3. Casos de estudio 2

3.1. Condiciones climáticas . . . . . . . . . . . . . . . . 25

3.2. Estructura y uso de los casos de estudio . . . . . . . . . . . 26

4. Metodología 31

4.1. Monitorización por plataforma web: Equs . . . . . . . . . . . 33

4.2. Modelado de los edificios: TRNSYS . . . . . . . . . . . . . . . 34

4.2.1. Definición de los modelos en programa de simulación: TRNBuild ...................... 35

4.2.2. Simulación de los modelos en TRNSYS Studio . . . . . . . . 39

4.3. Estimación del PMV y PPD . . . . . . . . . . . . . . . . 40 40

4.4. Análisis de sensibilidad mediante el método Monte Carlo . . . . . . 41

4.4.1. Análisis de sensibilidad mediante el uso de distribución uniforme ....................... . 42

4.4.2. Análisis de sensibilidad mediante el uso de distribución normal ..................... 43

4.5. Cambio de categoría y temperatura de consigna . . . . . . . . . . 43

5. Resultados y discusión $\quad 45$

5.1. Monitorización en tiempo real: Equs . . . . . . . . . . . . . 45

5.2. Validación de simulación en TRNSYS . . . . . . . . . . . . . . . 47

5.3. Análisis de sensibilidad mediante el método de Monte Carlo . . . . 50

5.3.1. Distribución uniforme . . . . . . . . . . . 50

5.3.2. Distribución normal . . . . . . . . . . . 52

5.4. Demandas energéticas e impacto económico . . . . . . . . . 55

$\begin{array}{lr}\text { 6. Conclusiones } & 57\end{array}$

Bibliografía $\quad \mathbf{5 9}$

$\begin{array}{lr}\text { Anexos } & 69\end{array}$

Anexo I: Estudio de caso: edificio en República Dominicana 69

$\begin{array}{ll}\text { Anexo 2: Código PMV y PPD } & \mathbf{8 5}\end{array}$

Anexo 3: Código PMV y PPD $\quad 89$ 


\section{Índice de figuras}

2.1. TRNSYS type y topología de red [79] . . . . . . . . . . . . 19

3.1. Planos arquitectónicos del primer caso de estudio: (a) planta baja; (b) primer piso. Geometría del modelo numérico: (c) vista noreste y (d) vista suroeste. . . . . . . . . . . . . . . 26

3.2. Planos arquitectónicos del segundo caso de estudio: (a) planta baja;

(b) primer piso; (c) segundo piso. Geometría del modelo numérico:

(d) vista noreste. . . . . . . . . . . . . . . . . . . 27

3.3. Geometría del modelo numérico en el software de diseño gráfico SketchUp $\_\ldots \ldots \ldots \ldots \ldots . \ldots \ldots$

4.1. Esquema de la metodología utilizada. . . . . . . . . . . . . 32

4.2. Captura de pantalla de la plataforma web "Equs" (http://equs-app.com). 34

4.3. Estimación computacional de PMV y PPD basada en ISO 7730 [44]. 40

5.1. Temperaturas internas y externas medidas por la plataforma web Equs. En el caso de la temperatura exterior, observamos que las temperaturas frías y calientes están entre $2^{\circ} \mathrm{C}$ y $41^{\circ} \mathrm{C}$, aproximadamente. La temperatura promedio fue de $15.49^{\circ} \mathrm{C}$. . . . . . . . . 46

5.2. Comparación entre los datos reales obtenidos con Equs y los resultados de simulaciones teóricas del primer caso de estudio: (a) para el mes de junio y (b) un solo día de junio. . . . . . . . . . . . . . .

5.3. Demanda de energía del edificio (caso España) en la configuración inicial de los parámetros de confort térmico. . . . . . . . . . .

5.4. Cambio del nivel de confort térmico en base al análisis de sensibilidad del factor de vestimenta en $P M V$ (factor de actividad permanece constante).

5.5. Cambio del nivel de confort térmico en base al análisis de sensibilidad del factor metabólico en PPD (factor de vestimenta permanece constante) 
5.6. Generación de sucesos distribuidos uniformemente para los parámetros de factor de vestimenta (clo) y factor metabólico (met). . . .

5.7. Generación de sucesos con base a distribución normal para los parámetros de factor de vestimenta (clo) y factor metabólico (met). . .

5.8. Relación en base al análisis de sensibilidad del factor de vestimenta $\left(\sigma_{c l o}\right):\left(\right.$ a) $\sigma_{P M V}$ y $\left(\right.$ b) $\sigma_{P P D} \ldots \ldots \ldots \ldots \ldots$

5.9. Relación en base al análisis de sensibilidad del factor metabólico $\left(\sigma_{\text {met }}\right)$ : (a) $\sigma_{P M V}$ y $\left(\right.$ b) $\sigma_{\text {PPD }} \ldots \ldots \ldots \ldots \ldots$

5.10. Relación entre las variaciones del factor de vestimenta (clo) y factor de actividad (met) y las anchuras de PMV. . . . . . . . . . . . 54

5.11. Relación entre las variaciones del facto de vestimenta (clo) y el factor metabólico (met) y las anchuras de PPD . . . . . . . . 55

5.12. Demanda de energía para varios valores de factor de vestimenta. . . 56

1. Ubicación y orientaciones del caso de estudio (República Dominicana). . . . . . . . . . . . . . . . 70

2. Imágenes reales del edificio y cafetería en República Dominicana. 70

3. Geometría del modelo numérico en el software de diseño gráfico SketchUp. . . . . . . . . . . . . . . . 71

4. Temperatura anual: ambiente. . . . . . . . . . . . . 78

5. Temperatura anual: zona del comedor. . . . . . . . . . . . 79

6. Temperatura anual: zona de la cocina. . . . . . . . . . . . . . . . 80

7. Temperatura anual: zona del depósito . . . . . . . . . . . . . . . . 81

8. Temperatura anual: zona del cuarto frío . . . . . . . . . . . . 81

9. Temperatura del mes de enero. . . . . . . . . . . . . . . . . 82

10. Temperatura del mes de agosto. . . . . . . . . . . . 83 


\section{Índice de tablas}

2.1. Valores de iluminación [32]. . . . . . . . . . . . . . . . 11

2.2. Niveles sonoros continuos equivalentes de ruido aéreo [40]. . . . . . 12

3.1. Propiedades térmicas y estructurales en ventanas. . . . . . . . . . . 28

3.2. Distribución de los casos de estudio. . . . . . . . . . . . . . . . . 29

3.3. Propiedades térmicas y estructurales de soluciones constructivas adoptadas. . . . . . . . . . . . . . . 30

4.1. Tipos de horarios en los edificios. . . . . . . . . . . . 36

4.2. Infiltraciones y ventilación para cada zona en verano e invierno. . . 36

4.3. Datos de entrada de ganancias internas en la zona de AC en el caso de España. . . . . . . . . . . . . . . . . . . . 37

4.4. Datos de entrada de ganancias internas en el caso de México. . . . . 38

4.5. Datos de entrada de confort en los dos edificios. . . . . . . . . . . . 38

4.6. Rango de temperatura de aire recomendado para oficinas y aulas (ISO 7730) [44]. . . . . . . . . . . . . . . . . . . 44

5.1. Demanda acumulada de energía entre junio y septiembre, con valores variables de clo y met, para diferentes temperaturas. . . . . . . 56

1. Orientaciones de la fachada. . . . . . . . . . . . . . . . 70

2. Área y volumen para las zonas del caso de estudio. . . . . . . . . . 72

3. Propiedades térmicas y estructurales. . . . . . . . . . . . . . 72

4. Propiedades térmicas y estructurales en ventanas. . . . . . . . . 73

5. Estructura de la fachada de la cocina. . . . . . . . . . . . . 73

6. Horario. . . . . . . . . . . . . . . . . . . . 74

7. Escala de porcentaje. . . . . . . . . . . . . . . 74

8. Distintos horarios de uso para el caso de República Dominicana. . . 75

9. Horario semanal. . . . . . . . . . . . . . . . 76

10. Caudal mínimo de ventilación para zona comedor y cocina. . . . . . 76

11. Datos de entrada de ganancias internas. . . . . . . . . . . . 76

12. Actividad realizada por los ocupantes en el comedor. . . . . . . . 76 
13. Actividad realizada por los ocupantes en la cocina. . . . . . . . . . . 77

14. Valores de confort para la cocina. . . . . . . . . . . . . 77

15. Datos de entrada de confort para comedor. . . . . . . . . . . . 77

16. Demanda acumulada de energía en el mes de enero, con valores variables de clo y met, para una temperatura de consigna de $25^{\circ} \mathrm{C} . \quad .83$ 


\section{Capítulo 1}

\section{Introducción}

\subsection{Introducción}

El consumo de energía relacionado con el sector de la construcción constituye una parte importante del consumo total de energía en todo el mundo (tan solo el $25.4 \%$ corresponde al sector residencial del gasto final de energía en la Unión Europea en 2015) y contribuye a más del $30 \%$ las emisiones de dióxido de carbono $\left(\mathrm{CO}_{2}\right)$ [1,2]. Es por ello que las edificaciones representan un objetivo clave para la mejora de la eficiencia energética global. En este contexto, el análisis energético puede proporcionar valiosa información cuantitativa sobre las cargas anuales del edificio y otras métricas de rendimiento para aquellos diseñadores que intenten cumplir con las normas establecidas a nivel gubernamental. Sin embargo, la comunidad de diseño de edificios aún carece de herramientas prácticas para llevar a cabo una evaluación rigurosa de la eficiencia energética durante la fase de diseño conceptual. Como resultado, el diseño de las edificaciones que buscan promover el rendimiento energético se hace a menudo en ausencia de estimaciones basadas en modelos matemáticos. En este proyecto se busca diseñar un modelo que permita realizar el estudio energético de edificios, basado en distintas herramientas de simulación y optimización, para obtener como resultado edificios más eficientes.

\subsection{Hipótesis}

El funcionamiento de la economía mundial se basa en el consumo de energía. Gracias a que se dispone de un suministro adecuado y suficiente de energía, las sociedades desarrolladas disfrutan de un elevado nivel en calidad de vida. Es por ello que si se consumen enormes cantidades de energía de manera poco eficiente puede conllevar al agotamiento de las reservas de energías no renovables y a la depen- 
dencia energética extrema. Por tanto, hoy en día se buscan diferentes alternativas de estudio para conocer y reducir el consumo energético. Una de ellas es el estudio de los edificios mediante simulaciones dinámicas; actualmente existen diversos softwares que nos permiten realizar esta tarea, como por ejemplo: Energy Plus $₫$,

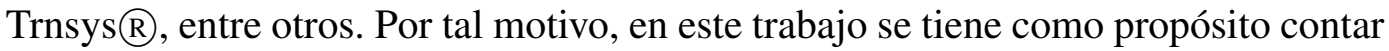
con estudios fiables de los edificios a estudiar mediante un software de simulación comparados con mediciones reales, que describan de manera eficaz el comportamiento del edificio en el ámbito energético. En la simulación del comportamiento de un edificio influyen una gran cantidad de variables a introducir y algunas de ellas dependen de la decisión subjetiva por parte del diseñador; por tal motivo existen estudios que nos permiten conocer las variables que mayor impacto producen en el consumo del edificio, como el análisis de sensibilidad, del cual esperamos obtener el impacto de al menos 2 variables en el confort térmico de los ocupantes.

\subsection{Objetivos}

En la presente sección se presentan los objetivos específicos y generales de la tesis. En los apartados precedentes hemos visto, por una parte, el problema global del consumo energético producto del sector de los edificios, sus posibles consecuencias y la interrelación que tiene con las emisiones de $\mathrm{CO}_{2}$. Este es el problema global Tierra/Humanidad que tratamos. Así mismo, hemos expuesto una alternativa de estudio, mediante herramientas de simulación dinámica y su verificación por medio de bases de datos reales. Así, para resumir los objetivos de este estudio diríamos que intentamos dar pasos significativos en la dirección del entendimiento y el tratamiento de este problema en sí mismo, desde el punto matemático y económico. Los objetivos que pretende conseguir la presente Tesis Doctoral se especifican a continuación:

\subsubsection{Objetivo general}

Este trabajo de tesis se desarrolla con el propósito de obtener un estudio fiable en edificaciones reales de diferentes tipologías, basado en modelos matemáticos y una plataforma de gestión energética implementados en un software de simulación dinámica, para conocer su comportamiento térmico y energético, permitiendo identificar potenciales medidas de ahorro energético y económico. Además, mediante un análisis estadístico-numérico, cuantificar el impacto de introducir de manera subjetiva, por parte del diseñador, las variables que influyen en la simulación. Estos estudios se esperan implementar en diversos edificios en países como España y México, en instalaciones residenciales, industriales y comerciales, entre otras. 


\subsubsection{Objetivos específicos}

- Construir una base de datos con modelos simples de geometría y materiales asociados a los casos de estudio.

- Implementar la monitorización a través de gestores energéticos, sobre un conjunto de edificios con ciertas características dependiendo de su tipología, que nos permita conocer las características energéticas del edificio.

- Realizar estudios energéticos mediante la aplicación de modelos matemáticos y programas de simulación de edificios con la finalidad de identificar las demandas energéticas y, a su vez, evaluar el ahorro en instalaciones.

- Realizar una comparación entre los resultados de la herramienta de simulación y el análisis real de los edificios a estudiar.

- Efectuar un análisis de sensibilidad de las variables físicas que afectan al desempeño energético de las edificaciones mediante el método Monte Carlo y, en específico, a la sensibilidad de la decisión subjetiva que toma el diseñador al establecer parámetros de confort.

- Realizar una evaluación de las características energéticas del modelo de edificación en base a los resultados obtenidos en el análisis de sensibilidad.

\subsection{Motivación}

El desarrollo del tema de investigación de esta tesis responde a cubrir una de las principales necesidades a nivel mundial: reducir de manera significativa el consumo energético y las emisiones de $\mathrm{CO}_{2}$.

En países como México, el tema energético se ha convertido en prioridad nacional con el propósito de brindar oportunidades para el desarrollo económico y la mejora de las condiciones de la población. Enfocar acciones y políticas para desarrollar nuevas tecnologías e investigaciones en esta área es una de las medidas estratégicas implementadas para la transición hacia un modelo sustentable y de uso racional de los recursos energéticos en el país. En este contexto, la reciente admisión de México como país miembro de la Agencia Internacional de Energía es una muestra de los recientes esfuerzos realizados, que conlleva a respetar los estándares estadísticos más exigentes a nivel mundial.

Con el propósito de desarrollar investigación que permita reducir los consumos energéticos y económicos en los edificios estudiados, garantizando a su vez la comodidad del ocupante, se han desarrollado estancias en México y España, lo que 
nos ha permitido obtener bases de datos de edificios, así como una formación más amplia en el área de la eficiencia energética de edificaciones.

Es una gran motivación impulsar el desarrollo de edificios eficientes que permitan obtener ahorros energéticos en lugares estratégicos como México, ya que es un país que tiene un enorme desarrollo tecnológico-industrial y que trabaja para estar a la vanguardia en estas áreas.

\subsection{Estructura de la tesis}

A continuación se da una breve descripción de cada capítulo que conforma esta tesis:

- Capítulo 1: en este primer capítulo se da una introducción al trabajo realizado en esta tesis, dando a conocer la relevancia del tema y la motivación de ésta; además, se incluyen otros puntos como: planteamiento del problema, objetivos y metodología.

- Capítulo 2: en este capítulo se da a conocer el estado del arte en relación al tema de tesis, con el objetivo de establecer un marco de referencia que nos permita interpretar los resultados y conclusiones.

- Capítulo 3: en esta sección se dan a conocer los casos de estudio del trabajo, donde se describen sus características principales como climatología y estructuras.

- Capítulo 4: en este se muestra la metodología propuesta para el desarrollo del proyecto, que consiste en obtener los rendimientos térmicos de los edificios y el análisis de sensibilidad para los parámetros subjetivos en el confort térmico, con el objetivo de identificar cuál de ellos tiene mayor influencia en el modelo.

- Capítulo 5: en él se muestran los resultados obtenidos de los estudios realizados; la verificación de la simulación térmica y las demandas de energía a partir del análisis de sensibilidad.

- Capítulo 6: finalmente, se presentan las conclusiones generales de la tesis, mediante un resumen de los resultados. Además, se incluyen propuestas a realizar como trabajo futuro.

Se incluyen algunas secciones adicionales como: lista de figuras y tablas, referencias y anexos. 


\subsection{Metodología de la tesis}

El proceso de investigación para esta tesis incluye cuatro etapas:

- La etapa inicial, con la exploración del marco teórico en el que se encuadra la investigación y el resumen de los antecedentes del problema a estudiar.

- Para la siguiente etapa se establece el software de simulación energética con el que se trabajará, además, se le incorporan algunos modelos matemáticos que complementan los estudios energéticos de una serie de edificios.

- En la tercera etapa se implementará un análisis de sensibilidad para determinar las variables que tengan mayor contribución al desempeño energético y al confort del edificio. A partir de los resultados obtenidos, se planea optimizar el modelo antes diseñado.

- La última etapa tiene como finalidad un análisis de los datos recopilados mediante cálculos, tablas y gráficas en las cuales podamos observar los resultados obtenidos de manera sencilla, con el propósito de desarrollar esta memoria de tesis que muestre el trabajo realizado y donde se establezcan las conclusiones de la tesis. 



\section{Capítulo 2}

\section{Marco teórico}

En estos tiempos de crisis económica, energética y medioambiental, el ahorro y la eficiencia energética aparecen como una excelente vía desde el ámbito energético para responder a estos tres desafíos. El reducir el consumo de energía permite el ahorro de nuestros recursos económicos, pospone el agotamiento de nuestros escasos recursos fósiles (de los que, sin embargo, depende mayoritariamente nuestro suministro energético) y, por último, parece revelarse como una de las mejores alternativas para reducir las emisiones de $\mathrm{CO}_{2}$. La clave para la existencia de estos ahorros reside en el hecho de que no consumimos energía, sino servicios energéticos: por tanto, puede ser posible proveer el mismo nivel de servicio energético con un menor nivel de consumo de energía.

Si bien el ahorro energético no es crítico para la resolución de todos los problemas ambientales, sí es cierto que su contribución a algunos de ellos, como el cambio climático, es muy significativa.

El último Informe de Evaluación del Panel Internacional sobre Cambio Climático (IPCC), informa que la temperatura promedio global de la superficie terrestre y oceánica combinada aumentó en $0.85^{\circ} \mathrm{C}$ durante el período de 1880 a 2012 y que es muy probable que la influencia humana haya sido la causa dominante de este calentamiento observado desde mediados del siglo XX [3].

\subsection{Introducción}

La envolvente del edificio es la interfaz entre el entorno exterior y el interior de los edificios, su función principal es actuar como una barrera física para proporcionar un espacio confortable para diferentes actividades, a costa de una cierta demanda de energía [4-9]. 


\subsection{Consumo energético del sector de los edificios}

Actualmente, la reducción del consumo energético asociado al uso y construcción de los edificios y el incremento de la eficiencia energética en el acondicionamiento climático de los mismos, son cuestiones que están recibiendo una gran atención, tanto desde el punto de vista científico-técnico como desde el empresarial [10].

El impacto producido por el consumo total de energía final en el sector de edificios, con un $32 \%$, es una de las principales causas del agotamiento de la capa de ozono, calentamiento global y cambio climático $[11,12]$. Los edificios representan cerca del $40 \%$ y del $17 \%$ del consumo de energía primaria en el mundo y de emisiones de $\mathrm{CO}_{2}$, respectivamente [13-16]. Además, los edificios residenciales a menudo son citados como una de las áreas más rentables para reducir el consumo de energía $[17,18]$.

\subsection{Niveles de ambiente interior}

La mayor parte del tiempo, las personas realizan sus actividades cotidianas en el interior de edificios, siendo por tanto preciso controlar las condiciones de confort (térmico, visual y calidad de aire) en el interior de los mismos e intentando minimizar el consumo energético simultáneamente.

Distintas formas de interpretar las condiciones ambientales han llevado al desarrollo de conceptos tales como edificio enfermo, calidad del aire o calidad ambiental interior, todos ellos encaminados a entender la complejidad de los contaminantes en los ambientes cerrados y los riesgos sobre la salud de la población.

A principios de la década de 1900, los activistas en los EE. UU. argumentaron que los niños debían estudiar al aire libre, que el aire fresco era esencial para el desarrollo de cuerpos y mentes saludables [19]. Por el contrario, quienes diseñan y definen oficinas con aire acondicionado ahora concluyen que la productividad y la comodidad se pueden lograr limitando la exposición al aire libre [20].

Además, los niveles adecuados de ambiente interior definidos por el confort térmico, confort acústico, confort de iluminación y la calidad del aire interior son esenciales para mejorar la salud y el bienestar de los ocupantes [20]. Cuando falta la calidad óptima de uno o más de estos cuatro parámetros, pueden surgir problemas como enfermedades, bajos niveles de producción e insatisfacción.

Es cierto que las personas mueren si se ponen demasiado calientes, frías, húmedas o secas, pero también es cierto que las personas han informado que se sienten cómodas a temperaturas que oscilan entre 6 y $30^{\circ} \mathrm{C}[21,22]$. 


\subsubsection{Calidad del aire}

Existe un término relativamente nuevo en el área de la edificación denominado el síndrome del edificio enfermo, el cual se puede considerar como sinónimo de una calidad de aire pobre, lo que, a su vez, se traduce en la posible aparición de problemas de salud y en la falta de confort para los usuarios [23]. Por este motivo, la calidad del aire se debe definir en función de las necesidades de los ocupantes, las cuales se pueden resumir en la percepción de aire fresco en lugar de aire cargado o viciado y que los riesgos para la salud que se pudieran derivar de respirar dicho aire sean despreciables [24].

Generalmente, las sustancias contaminantes responsables de una calidad de aire pobre en el interior de los edificios son: los olores, el dióxido de carbono, el humo de tabaco, los óxidos de nitrógeno y los aerosoles [23]. Sin embargo, para estimar el volumen de aire necesario para conseguir una calidad de aire óptima, se utiliza la concentración de dióxido de carbono $\left(\mathrm{CO}_{2}\right)$ [25], cuya principal fuente de generación es la respiración humana. La cantidad de $\mathrm{CO}_{2}$ producida por el ser humano está directamente relacionada con la actividad física que esté realizando, siendo posible estimar la tasa de generación de $\mathrm{CO}_{2}$ en función de dicha actividad [26,27].

Para controlar los niveles de $\mathrm{CO}_{2}$ en el interior de un determinado edificio, la técnica más utilizada es la ventilación, que se puede definir como el aporte y/o extracción de aire en un cierto entorno, ya sea de una forma natural o mecánica [28]. Para describir la dinámica del $\mathrm{CO}_{2}$, se recomienda utilizar un modelo basado en balance de masas de la sustancia contaminante, en este caso el $\mathrm{CO}_{2}$, como se muestra en la siguiente ecuación [29]:

$$
V \cdot \frac{\partial C_{i}}{\partial t}=F_{o} \cdot C_{o}-F_{o} \cdot C_{i}+F_{f} \cdot C_{o}-F_{e} \cdot C_{i}+N_{p} \cdot G
$$

donde

- $V$ : volumen de aire del recinto estudiado $\left[\mathrm{m}^{3}\right]$.

- $C_{o}$ : concentración de $\mathrm{CO}_{2}$ en el aire exterior $\left[\mathrm{g} / \mathrm{m}^{3}\right]$.

- $\mathrm{C}_{i}$ : concentración de $\mathrm{CO}_{2}$ en el aire interior $\left[\mathrm{g} / \mathrm{m}^{3}\right]$.

- $F_{o}$ : flujo de aire exterior a través de ventilación forzada $\left[\mathrm{m}^{3} / \mathrm{s}\right]$.

- $F_{f}$ : flujo de aire por infiltración $\left[\mathrm{m}^{3} / \mathrm{s}\right]$.

- $F_{e}$ : flujo de aire por exfiltración $\left[\mathrm{m}^{3} / \mathrm{s}\right]$.

- $N_{p}$ : número de ocupantes [-].

- $G$ : tasa de generación de $\mathrm{CO}_{2}[\mathrm{~g} / \mathrm{s}$ por persona $]$. 
Por otro lado, en España existe otra normativa para estimar la calidad de aire interior. Esta norma se encuentra publicada en el estándar UNE-EN 1779 y en el informe 1752 del CEN (Comité Europeo de Normalización). Además, en el Reglamento de Instalaciones Térmicas en los Edificios [30] se específica cómo regular la calidad de aire interior en función del nivel de $\mathrm{CO}_{2}$, recomendándose que dicho nivel oscile entre 500 y 800 ppm (partes por millón). También se incluye la siguiente ecuación para calcular la tasa de ventilación forzada necesaria que permitiría eliminar la concentración de un determinado contaminante:

$$
C=\frac{q}{C_{a}-C_{i}} \cdot \frac{1}{E_{v}}
$$

- $C$ : caudal volumétrico de aire de impulsión $\left[\mathrm{m}^{3} / \mathrm{s}\right]$.

- $q$ : caudal másico de la sustancia contaminante medida $[\mathrm{mg} / \mathrm{s}]$.

- $C_{a}$ : concentración permitida en el ambiente $\left[\mathrm{mg} / \mathrm{m}^{3}\right]$.

- $C_{i}$ : concentración en el aire de impulsión $\left[\mathrm{mg} / \mathrm{m}^{3}\right]$.

- $E_{v}$ : eficacia de ventilación $[-]$.

La eficacia de ventilación $E_{v}$ se define como la relación $E_{v}=C_{e} / C_{r}$, siendo $C_{e}$ la concentración del contaminante en la extracción del aire y $C_{r}$ la correspondiente en la zona ocupada [24]. Por tanto, la eficacia de ventilación es función de la ubicación y de las características de los elementos de suministro, así como de la extracción del aire y de las fuentes de contaminación.

\subsubsection{Confort visual}

El ser humano percibe la mayor parte de la información a partir del sentido de la vista, por lo que la luz resulta un elemento muy importante, ya que permite distinguir la forma, el color y la perspectiva de los distintos objetos existentes en un determinado entorno [31]. Por tanto, el confort visual se puede expresar a través de niveles óptimos de iluminación, luminancia y color [32]. Por ejemplo, en la Tabla 2.1 se muestran los niveles de iluminación idóneos en función del recinto que se esté estudiando. Estos niveles se encuentran publicados en estándares [33-35]. 


\begin{tabular}{|c|c|}
\hline Función & Estancia (nivel de iluminación) [lux] \\
\hline \hline Doméstico & $\begin{array}{c}\text { Salón (50-150), dormitorio (50-100), } \\
\text { cocina (150-300), baño (150), aseo (100). }\end{array}$ \\
\hline Hotel & $\begin{array}{c}\text { Recepción (300), dormitorio (50-100), } \\
\text { habitaciones (100) }\end{array}$ \\
\hline Librería & General (300), estanterías de libros (150) \\
\hline Oficina & General (500), sala de conferencias (500) \\
\hline
\end{tabular}

Tabla 2.1: Valores de iluminación [32].

Las condiciones de confort visual en el interior de un recinto dependen de las propiedades geográficas y atmosféricas donde dicho recinto esté situado. Por lo tanto, el nivel de iluminación real en el interior del mismo se debe calcular teniendo en cuenta propiedades como el diseño de la ventana, su orientación, los coeficientes de reflexión, y otros [36]. El objetivo de hacer coincidir el nivel de iluminación real con el deseado (Tabla 2.1) a veces es imposible cumplir usando únicamente luz natural, por lo que suelen ser precisos mecanismos de iluminación artificial, véase [37].

\subsubsection{Confort acústico}

El confort acústico es el nivel de ruido que se encuentra por debajo de los niveles permitidos que potencialmente causan daños a la salud y que, además, ha de ser aceptado como confortable por los trabajadores afectados [38]. El confort acústico es el nivel sonoro que no molesta, que no perturba y que no causa daño directo a la salud.

El disconfort produce efectos extra auditivos que son variados y entran dentro del campo de la ergonomía. Éstos pueden ser:

- Subjetivos: el efecto indeseable del ruido es el más común, ya que un mismo ambiente acústico puede ser desagradable para una persona y para otra no.

- Conductuales: el disconfort acústico afecta al comportamiento de los trabajadores, por perturbar el rendimiento en el trabajo y la comunicación entre trabajadores, y siempre se manifiesta como queja directa de éstos.

- Psicofisiológicos: el ruido produce variaciones en la frecuencia cardíaca, aumento de la presión sanguínea, contracciones musculares, efectos sobre el sueño, etc.

La principal fuente de contaminación acústica en la ciudades proviene de las redes de movilidad, concretamente del tráfico rodado. Su unidad de medida inter- 
nacional es el decibelio (dBA). Una calle se puede considerar confortable si el nivel entre las 6 y 22 horas no supera $\operatorname{los} 65 d B A$. Aquellas calles con un nivel sonoro superior se consideran como críticas.

El nivel sonoro continuo equivalente ( $L A e q$ ), es el nivel en $d B A$ de un ruido de nivel constante hipotético correspondiente a la misma cantidad de energía sonora que el ruido real considerado, durante un período de tiempo $\mathrm{T}$.

En el Tabla 2.2 se muestran los niveles sonoros continuos equivalentes ( $L A e q)$ de ruido aéreo, que se recomienda no sobrepasar en los locales [39, 40].

\begin{tabular}{|c|c|c|}
\hline Tipo de edificio & Local & LAeq $(\mathrm{dBA})(8-22 \mathrm{~h})$ \\
\hline \hline \multirow{2}{*}{ Residencial (público y privado) } & Zonas de estancia & 45 \\
\cline { 2 - 3 } & Dormitorios & 40 \\
\hline \multirow{2}{*}{ Administrativo y de oficinas } & Despachos profesionales & 40 \\
\cline { 2 - 3 } & Oficinas & 45 \\
\hline \multirow{2}{*}{ Sanitario } & Zonas de estancia & 45 \\
\cline { 2 - 3 } & Dormitorios & 30 \\
\hline \multirow{2}{*}{ Docente } & Aulas & 40 \\
\cline { 2 - 3 } & Salas de lectura & 35 \\
\hline
\end{tabular}

Tabla 2.2: Niveles sonoros continuos equivalentes de ruido aéreo [40].

Existen dos técnicas que, adecuadamente combinadas, permiten crear un ambiente acústico de confort en el interior de un recinto:

- El acondicionamiento acústico: mediante la utilización de determinados materiales se puede incrementar la absorción acústica de un recinto, reduciendo con ello el sonido reflejado por los límites del local. El resultado es una reducción del nivel de ruido.

- El aislamiento acústico: utilizando materiales aislantes, podemos reducir la transmisión de ruido entre dos locales colindantes o entre el exterior y el recinto que tratamos de proteger.

\subsubsection{Confort térmico}

El confort térmico es una de las características más importantes cuando se estima el ambiente interior; ha sido definido en paralelo por ASHRAE (American Society of Heating, Refrigerating and Air Conditioning Engineers) Standard 55 e ISO (International Standard Organization) Standard 7730 como la condición de la mente en la que la satisfacción se expresa con el entorno térmico [41-45]. Sin embargo, esta 
definición se puede considerar ambigua ya que se deja abierto el significado de condición de la mente, como el de satisfacción, pero lo que sí se deduce correctamente es que el juicio del confort es un proceso cognitivo influido por distintos tipos de procesos, entre los que se incluyen físicos, fisiológicos o incluso psicológicos [46].

Las expectativas de confort dependen de varias circunstancias, como por ejemplo, el lugar donde se encuentre el ser humano, los motivos que hacen que se encuentre en dicho lugar, la época del año, etc. Sin embargo, según diversos estudios, aunque los climas, las condiciones de vida y las culturas difieran bastante a lo largo del mundo, la temperatura que la gente elige para el confort bajo condiciones similares de vestimenta, actividad, humedad y velocidad de aire es muy parecida [46].

Una búsqueda rápida en bases de datos científicas muestra que la comodidad en los edificios es una temática de interés en campos de investigación muy diferentes como Ingeniería (39\%), Energía (14\%), Ambiental (10\%), Informática (7.5\%) y Ciencias Sociales (7\%), en menor medida, se encuentra en ciencia de la salud, la tierra y economía. Algunos ejemplos de análisis del confort térmico son el diseño de una sinagoga [47], estrategias para viviendas de bajo costo [48], materiales para almohadillas protectoras de la cadera [49] y evaluación en oficinas modernas de bajo consumo de energía [50].

Por lo tanto, los rangos de comodidad dependerán de los parámetros físicos y humanos de sus ocupantes y, por lo tanto, deben establecerse de acuerdo con la especificidad de cada región o país [51]. Estándares internacionales bien establecidos de países y regiones como EE. UU. o Europa, restringen los intervalos de comodidad basados en índices numéricos, como el voto medio pronosticado (PMV) y el porcentaje predicho de insatisfacción (PPD), ambos definidos en la normativa ISO $7730[42,52,53]$. Sin embargo, muchos países tienen pocos o incluso ningún estándar de confort térmico propio o estudios de campo asociados a la sensación térmica y la adaptabilidad. México es un ejemplo de esta situación, ya que solo hay dos modelos disponibles: un estándar ha sido desarrollado para la ciudad de Hermosi1lo, noroeste de México (condiciones cálidas y extremadamente secas) [54,55] y el otro para las cuatro principales regiones climáticas de México en las temporadas de enfriamiento y calefacción [51].

Los índices PMV y PPD se utilizan para predecir la sensación térmica en función de los cuatro parámetros ambientales térmicos clásicos (temperatura del aire, temperatura radiante media, velocidad del aire y humedad) y dos parámetros humanos subjetivos: actividad y vestimenta; donde factor de actividad o metabólico se define como la intensidad de las tareas físicas que desarrolla una persona expresada a través del índice de tasa metabólica (met) y el factor de vestimenta ( $\mathrm{clo}$ ) representa la resistencia térmica total desde la piel a la superficie exterior del cuerpo vestido. Esta comodidad es aún más significativa cuando el edificio se localiza en 
condiciones extremas como el norte de Europa y el norte de Asia (condiciones de frío) $[56,57]$ y países tropicales como Camerún, Singapur y México (condiciones cálidas) [58-60]. Por otro lado, el uso de sistemas HVAC (Calefacción, Ventilación y Aire Acondicionado) está fuertemente relacionado con el confort térmico y el consumo de energía y es bien sabido que, en países con condiciones climáticas extremas, los sistemas HVAC pueden representar más de la mitad de la demanda total de energía de una sola vivienda $[61,62]$.

\section{Nota}

1 unidad metabólica $=1[$ met $]=58,2\left[\mathrm{~W} / \mathrm{m}^{2}\right]$;

1 unidad de ropa $=1[\mathrm{clo}]=0,155\left[\mathrm{~m}^{2} \cdot{ }^{\circ} \mathrm{C} / \mathrm{W}\right]$.

\section{4. Índices PMV y PPD}

El PMV es un índice que refleja el valor medio de los votos emitidos por un grupo numeroso de personas respecto de una escala de sensación térmica de 7 niveles, basado en el equilibrio térmico del cuerpo humano. El equilibrio térmico se obtiene cuando la producción interna de calor del cuerpo es igual a su pérdida hacia el ambiente. En un ambiente moderado, el sistema termorregulador tratará de modificar automáticamente la temperatura de la piel y la secreción de sudor para mantener el equilibrio térmico. El PMV puede ser calculado para diferentes combinaciones de tasa metabólica, aislamiento de la ropa, temperatura del aire, temperatura radiante media, velocidad relativa del aire y humedad del aire. El PMV predice el valor medio de los votos sobre la sensación térmica que emitiría un grupo numeroso de personas sometidas al mismo ambiente. No obstante, los votos individuales están dispersos alrededor del valor medio, siendo útil el poder estimar el número de personas que, probablemente, sentirán incomodidad debida al calor o al frío [44].

EL PPD es un índice que establece una predicción cuantitativa del porcentaje de personas que se sentirán insatisfechas por notar demasiado frío o demasiado calor. Para el propósito de esta norma internacional, las personas térmicamente insatisfechas son aquellas que votarán muy caluroso, caluroso, fresco o frío, sobre la escala de niveles de sensación térmica [44].

El cálculo del PMV puede realizarse a partir de las ecuaciones (1) a (4), adicionalmente en el apartado de metodología y anexos se muestra el código computacional que permite el cálculo de ambos indices en función de diferentes parámetros: 


$$
\begin{aligned}
& P M V=[0.303 \cdot \exp (-0.036 \cdot M)+0.028] \\
& \left\{(M-W)-3.05 \cdot 10^{-3} \cdot\left[5733-6.99 \cdot(M-W)-P_{a}\right]\right. \\
& -0.42 \cdot[(M-W)-58.15]-1.7 \cdot 10^{-5} \cdot M \cdot\left(5867-P_{a}\right) \\
& -0.0014 \cdot M \cdot\left(34-t_{a}\right)-3.96 \cdot 10^{-8} \cdot f_{c l} \text {. } \\
& \left.\left[\left(t_{c l}+273\right)^{4}-\left(\overline{t_{r}}+273\right)^{4}\right]-f_{c l} \cdot h_{c} \cdot\left(t_{c l}-t_{a}\right)\right\} \\
& t_{c l}=35.7-0.028 \cdot(M-W)-I_{c l} \\
& \left\{3.96 \cdot 10^{-8} \cdot f_{c l} \cdot\left[\left(t_{c l}+273\right)^{4}-\left(\overline{t_{r}}+273\right)^{4}\right]+f_{c l} \cdot h_{c}\left(t_{c l}-t_{a}\right)\right\} \\
& h_{c}= \begin{cases}2.38 \cdot\left|t_{c l}-t_{a}\right|^{0.25} & \text { para } 2.38 \cdot\left|t_{c l}-t_{a}\right|^{0.25}>12.1 \cdot \sqrt{V_{a r}} \\
12.1 \cdot \sqrt{V_{a r}} & \text { para } 2.38 \cdot\left|t_{c l}-t_{a}\right|^{0.25}<12.1 \cdot \sqrt{V_{a r}}\end{cases} \\
& f_{c l}= \begin{cases}1.00+1.290 I_{c l} & \text { para } I_{c l} \leq 0.078 m^{2} \cdot K / W \\
1.05+0.645 I_{c l} & \text { para } I_{c l}>0.078 m^{2} \cdot K / W\end{cases}
\end{aligned}
$$

donde

- $M$ : tasa metabólica, en vatios por metro cuadrado $\left[W / m^{2}\right]$.

- $W$ : potencia mecánica efectiva, en vatios por metro cuadrado $\left[W / m^{2}\right]$.

- $I_{c l}$ : aislamiento de la ropa, en metros cuadrados kelvin por vatio $\left[m^{2} \cdot K / W\right]$.

- $f_{c l}$ : factor de superficie de la ropa.

- $t_{a}$ : temperatura del aire, en grados Celsius $\left[{ }^{\circ} \mathrm{C}\right]$.

- $\overline{t_{r}}$ : temperatura radiante media, en grados Celsius $\left[{ }^{\circ} \mathrm{C}\right]$.

- $V_{a r}$ : velocidad relativa del aire, en metros por segundo $[\mathrm{m} / \mathrm{s}]$.

- $P_{a}$ : presión parcial del vapor de agua, en pascales $[P a]$.

- $h_{c}$ : coeficiente de transmisión del calor por convección, en vatios por metro cuadrado kelvin $\left[W / m^{2} \cdot K\right]$.

- $t_{c l}$ : temperatura de la superficie de la ropa, en grados Celsius $\left[{ }^{\circ} \mathrm{C}\right]$. 
Una vez determinado el valor del PMV, se calcula el PPD utilizando la ecuación (5),

$$
P P D=100-95 \cdot \exp \left(-0.03353 \cdot P M V^{4}-0.2179 \cdot P M V^{2}\right)
$$

Otra manera de realizar el cálculo es mediante las ecuaciones mostradas en el anexo 2.

\subsection{Simulación energética de edificios}

Hoy en día, los diseñadores necesitan herramientas que respondan a preguntas muy específicas, incluso durante la fase inicial de diseño [63]. Mediante el uso de diferente software de simulación de energía, los diseñadores pueden considerar opciones específicas (por ejemplo, calefacción y refrigeración). Además, pueden predecir el comportamiento térmico de los edificios antes de su construcción y simular los costos de energía en edificios existentes en sus condiciones actuales, estableciendo las mejores medidas de adaptación térmica para asegurar edificios eficientes y confortables. Además del consumo de energía, las herramientas de software de simulación también pueden usarse para calcular las siguientes variables:

- Temperaturas interiores.

- Temperaturas exteriores.

- Necesidades de consumo de los sistemas HVAC.

- Las necesidades de iluminación natural de los ocupantes.

- Confort interior de los habitantes.

- Niveles de ventilación.

Las herramientas de software de simulación de energía también pueden permitir considerar todas las regulaciones vigentes y, simultáneamente, proporcionar una sensación de confort a sus habitantes a través de un diseño correcto de los sistemas de calefacción y refrigeración. Dicho software también tiene herramientas disponibles para mejorar las soluciones constructivas a través de la simulación del efecto de la incorporación de sistemas solares pasivos en los edificios, como los sistemas de sombreado horizontal y vertical, así como un estudio más preciso del uso de las cargas del sistema HVAC [63].

Las simulaciones basadas en Building Information Modeling (BIM) se utilizan comúnmente como una herramienta efectiva para analizar simultáneamente el consumo de energía y el confort térmico en los edificios [64]. 
Existen diferentes programas de simulación de edificios basados en el estándar

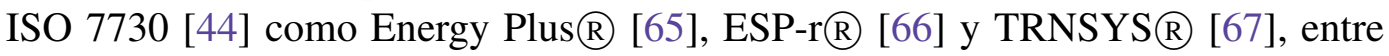
otros [68].

\subsubsection{Energy Plus $®$}

Energy Plus es una de las herramientas de software de simulación de energía más conocidas. Su desarrollo comenzó en 1996, patrocinado por el Departamento de Energía (DOE) de los Estados Unidos de América (EE. UU.) [69]. Inicialmente, el gobierno de EE. UU. estaba desarrollando dos herramientas de software diferentes, BLAST y DOE-2, que fueron abandonadas después de muchas discusiones y representaban un primer paso y la base de trabajo de Energy Plus. Energy Plus tiene las características y capacidades de BLAST y DOE-2; sin embargo, es una herramienta de software completamente nueva que combina el balance de calor de BLAST con un sistema HVAC genérico. Energy Plus tiene como objetivo desarrollar y organizar herramientas de software en módulos que pueden trabajar juntos o por separado. Es importante destacar que Energy Plus no tiene una interfaz visual que permita a los usuarios ver y diseñar el edificio. En este caso, se deben utilizar herramientas de software de terceros, es decir, Design Builder. Energy Plus es una herramienta de software de simulación térmica que permite el análisis de la energía en todo el edificio y la carga térmica, y es utilizada por ingenieros, arquitectos e investigadores para modelar el uso de energía y el uso del agua en los edificios. La herramienta de software simula modelos para calefacción, refrigeración, iluminación, ventilación, otros flujos de energía y uso del agua. La simulación de un edificio se divide en dos etapas $[65,69-71]$ :

- Construcción del edificio.

- Introducción de datos, como aspectos ambientales, efectos de sombreado, sistema de enfriamiento, ganancias internas, etc.

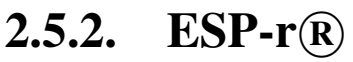

La herramienta de software de simulación de energía ESP-r está destinada a apoyar el proyecto de construcción con respecto a la energía y el desempeño ambiental de una manera realista y precisa. La herramienta es un software matemático destinada para diseñadores que coordina datos, simulación, aplicaciones CAD, diferentes herramientas para evaluar el rendimiento, etc. El ESP-r usa ecuaciones complejas para tratar todos los aspectos al mismo tiempo (geometría, construcción, operación, distribución, disipación de calor, etc.). Estas ecuaciones se integran en sucesivos pasos de tiempo en respuesta a las influencias de los ocupantes y los sistemas de control de clima. La geometría del edificio se puede establecer en herramientas de 
software de CAD u otras herramientas similares para permitir la especificación de la geometría de los edificios $[66,72]$.

El tiempo de simulación del edificio utilizando la herramienta de simulación ESP-r puede variar en un rango de un minuto a una hora. Las salidas de las simulaciones se pueden ver por las interacciones entre los dominios de evaluación o se pueden exportar a otro software de gráficos. ESP-r es extremadamente útil y es una herramienta poderosa para simular muchas tecnologías innovadoras. Sin embargo, el programa requiere un gran conocimiento y experiencia por parte de sus usuarios y requiere un largo proceso de aprendizaje.

\subsubsection{TRNSYS $\mathbb{R}$}

TRNSYS (TRaNsient SYStems simulation Program) es una herramienta de simulación gráfica flexible basada en el lenguaje FORTRAN, desarrollada en 1975 por la Universidad de Wisconsin. Si bien la mayoría de las simulaciones se centran en el rendimiento de los sistemas térmicos eléctricos y energéticos, TRNSYS también puede utilizarse para modelar otros sistemas dinámicos, como el flujo de tráfico o los procesos biológicos [67,73-75]. Estudios particulares que usan TRNSYS y se reportan en la literatura son: la comparación de estrategias de enfriamiento para una oficina en diferentes climas europeos [76], confort térmico en una piscina cubierta [77], comportamiento de la operación en modo de enfriamiento de un edificio de energía casi nula en China [78], entre otros.

\section{TRNSYS Studio y TRNBuild}

TRNSYS es una herramienta de software de simulación de sistemas transitorios con una estructura modular que ha sido especialmente diseñada para desarrollar sistemas complejos relacionados con la energía, delineando el problema en una serie de componentes más pequeños [67]. Los componentes (types) pueden variar desde una bomba de calor simple a una zona múltiple de un complejo de edificios. Los componentes se configuran a través de la interfaz gráfica de usuario conocida como TRNSYS Simulation Studio. En la herramienta de software de simulación de energía TRNSYS, la construcción del edificio se puede lograr mediante la introducción de datos en la interfaz visual dedicada, conocida por TRNBuild [69].

Los componentes se pueden compartir entre múltiples usuarios sin tener que volver a compilar la herramienta de software debido al uso de la tecnología DLL [63]. Además, esta herramienta de software de simulación energética permite al usuario incorporar otros componentes desarrollados en herramientas de software como $\operatorname{MATLAB} \cap$, Microsoft Excel $\cap, \operatorname{VBA} \cap$, etc. 


\section{Funcionamiento TRNSYS}

Una simulación de TRNSYS se crea conectando modelos de componentes individuales (types) en un modelo de sistema completo. Estos types requieren parámetros, entradas y producen salidas. Los parámetros son constantes para toda la simulación, mientras que las entradas pueden ser constantes, basadas en ecuaciones ingresadas directamente en la simulación o conectadas a las salidas de otro type (Fig. 2.1).

Las rutinas del kernel de TRNSYS no hacen suposiciones sobre estas entradas y salidas o sobre los algoritmos contenidos en los types conectados en la simulación; para el kernel son modelos de caja negra [79]. El kernel maneja las entradas y hace uso de las ecuaciones de los types a las entradas y luego, en cada iteración, llama a lostypes cuyas entradas han cambiado hasta que se logra la convergencia. La decisión sobre si los valores de entrada han cambiado puede hacerse en función de una tolerancia relativa o absoluta, pero es la misma para todas las entradas independientemente de lo que represente esa entrada. En otras palabras, un valor de temperatura y un valor de humedad tendrían la misma tolerancia absoluta o relativa, aunque eso podría representar un acuerdo significativamente mayor en un caso que en el otro.
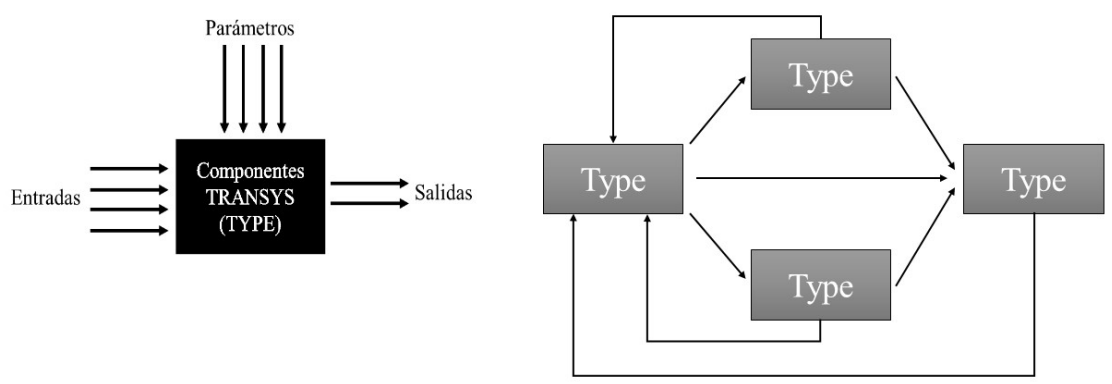

Figura 2.1: TRNSYS type y topología de red [79].

\subsection{Conceptos energéticos}

Entender el edificio como un sistema energético sin duda ayuda a simplificar la complejidad con la que las distintas instalaciones térmicas son capaces de mantenernos en razonables condiciones de confort térmico, sean cuales sean las condiciones exteriores. Si nos paramos a pensar en lo que esto implica, un sistema no es 
más que una cadena de elementos, un conjunto de componentes relacionados entre sí que deben su éxito a su funcionamiento en conjunto. Por ello el dimensionado de las instalaciones térmicas, ya sean de calefacción o refrigeración, comienza por las condiciones climatológicas de la localidad en la que nos ubiquemos y por las características constructivas del edificio, sin olvidarnos de completar la ecuación con la influencia que la actividad humana ejerce sobre el balance final de consumo energético, que en resumen determinará la emisión de distintos gases a la atmósfera.

En esta apartado se abordarán conceptos como energía, demanda, consumo o rendimiento, elementos básicos para poder adentrarnos posteriormente en el análisis de cada uno de los componentes del sistema energético de cualquier edificio [80,81].

\subsubsection{Energía}

Se define como energía la capacidad de los cuerpos o conjuntos de éstos para desarrollar un determinado trabajo. La unidad de medida de la energía en el sistema internacional es el Julio $(J)$. El julio está relacionado con el trabajo, y con la ley de conservación de la energía. El julio es la cantidad de energía necesaria para elevar un Newton $(N)$, un metro $(m)$ sobre la superficie terrestre, y también se mide en Kcal, cuando se define como la energía necesaria para elevar la temperatura de 1 Kilo $(K g)$ de agua, 1 grado $\left({ }^{\circ} C\right)$.

La potencia no es otra cosa que el ritmo al que se desarrolla ese trabajo, y por tanto incorpora la variable del tiempo, midiéndose en Julios/Segundo, o lo que viene a ser lo mismo, en Vatios (W).

Las formas de energía que se muestran en la naturaleza se pueden clasificar de la siguiente forma:

- Energía cinética: relacionada con el movimiento.

- Energía potencial: según la posición de una masa.

- Energía eléctrica: la que se obtiene con una diferencia de potencial eléctrico.

- Energía lumínica: la que tiene como origen las fuentes luminosas, como el fuego o el sol.

- Energía mecánica.

- Energía térmica: se manifiesta en forma de calor.

- Energía eólica: el viento y como aprovechar su fuerza.

- Energía solar: capta la radiación del sol. 
- Energía fotovoltaica: un tipo de energía solar que transforma la radiación solar en electricidad.

- Energía hidráulica o hidroeléctrica: aprovecha la diferencia de altura del agua en una presa.

- Energía mareomotriz: el mar como productor de energía.

- Energía biomasa: libera la energía de la materia vegetal.

- Energía geotérmica: aprovecha la diferencia de temperatura entre el interior de la tierra y su superficie.

- Energía nuclear: relacionada con las reacciones nucleares.

- Energía química: procedente de las reacciones químicas, por ejemplo el combustible de un motor.

- Energía sonora.

- Energía radiante.

- Energía de reacción.

- Energía iónica.

- Energía electromagnética.

- Energía metabólica.

- Energía magnética.

- Energía calorífica.

Llegados a este punto cabe destacar la diferencia entre energía primaria y energía final. La energía primaria es aquella que proviene de una fuente disponible en la naturaleza, mientras que la energía final es la que consumimos en nuestros edificios y que, por tanto, procede de la transformación de la energía primaria.

\subsubsection{Demanda de energía}

La demanda energética de un edificio es la necesidad de energía que necesita para calefactar o refrigerar sus espacios interiores para mantener el edificio en unas condiciones confortables. La demanda energética del edificio viene determinado por su su aislamiento, compacidad, tipología de ventanas, renovaciones de aire, cubiertas, suelos, etc. 


\subsubsection{Rendimiento energético}

La eficiencia energética o rendimiento energético surge del cociente entre la energía útil o utilizada por un sistema $\left(E_{\text {obtenida }}\right)$ y la energía total consumida (E $\left.E_{\text {consumida }}\right)$ :

$$
\eta=E_{\text {obtenida }} / E_{\text {consumida }}
$$

\subsubsection{Rendimiento térmico}

El rendimiento térmico de un edificio se refiere al proceso de modelado de la transferencia de energía entre un edificio y el entorno. La comprensión del rendimiento térmico de los edificios calcula la carga de refrigeración y, por lo tanto, ayuda a estimar la capacidad, el tamaño y la selección de un aparato de aire acondicionado. Para un edificio no acondicionado, calcula la variación de temperatura dentro de un edificio. Estos son muy esenciales y nos permiten determinar la efectividad del diseño del edificio. La carga de diseño se basa en las condiciones de diseño interior y exterior. Se basa en la estimación del enfoque de estado estable de varios elementos de construcción, como paredes, techos, puertas, etc., y en la estimación de la tasa de transferencia de calor general.

La energía producida o energía salida del sistema $\left(E_{\text {producida }}\right)$ es siempre menor que la energía suministrada o energía entrada al sistema $\left(E_{\text {suministrada }}\right)$. El rendimiento térmico o eficiencia de una máquina térmica es un coeficiente o ratio adimensional calculado como el cociente de la energía producida (en un ciclo de funcionamiento) y la energía suministrada a la máquina (para que logre completar el ciclo termodinámico). Se designa con la letra griega $\eta_{t e r}$ :

$$
\eta_{\text {ter }}=E_{\text {producida }} / E_{\text {suministrada }}
$$

\subsubsection{Consumo de energía}

El consumo de energía ya sea de calefacción, refrigeración o agua caliente sanitaria de un edificio para atender a la demanda de los usuarios, viene dado por una sencilla expresión:

Consumo Energético = Demanda de energía $/$ Rendimiento del Sistema

De esta fórmula podemos extraer como primera conclusión que para disminuir el consumo de energía en el edificio podemos actuar de distintas maneras:

- Disminuir la demanda de energía. 
- Aumentar el rendimiento del sistema.

- Actuar de forma conjunta sobre demanda y rendimiento del sistema.

\subsection{Análisis de sensibilidad}

Una posible definición del análisis de sensibilidad es la siguiente: el estudio de cómo la incertidumbre en la salida de un modelo (numérico o de otro tipo) puede asignarse a diferentes fuentes de incertidumbre en la entrada del modelo [82].

El análisis de sensibilidad (SA) generalmente se lleva a cabo junto con simulaciones de energía para comprender el rendimiento de los edificios y reducir sus consumos. La calidad de sus resultados depende principalmente de los modelos térmicos y los datos de entrada. Tener datos precisos sobre las propiedades y las condiciones de operación de los edificios es difícil. En consecuencia, los aportes de las simulaciones se ven afectados por incertidumbres que pueden tener efectos significativos en los productos y pueden ser importantes para considerar. Los SA garantizan la fiabilidad y versatilidad de los análisis realizados [83].

\subsection{Aplicación de herramientas de simulación y aná- lisis de sensibilidad}

Un enfoque interesante es el resultado de la unión de las herramientas de simulación de energía y el análisis de sensibilidad, para definir qué parámetros de entrada tienen más influencia en la salida [84]. Esta propuesta ayuda a definir los parámetros más apropiados a considerar, dependiendo del objetivo del modelado. Este tipo de análisis se puede hacer por métodos populares como Morris, regresión, métodos basados en la varianza y Monte Carlo, entre otros. El análisis de sensibilidad basado en el método Monte Carlo ofrece la opción de priorizar y fijar las entradas también en casos en que se consideran salidas múltiples $[85,86]$. Se pueden encontrar en la literatura diversos estudios con análisis de sensibilidad realizados sobre los efectos de los parámetros técnicos y físicos en el consumo de energía de los edificios y los programas de simulación [83,86-90]. 



\section{Capítulo 3}

\section{Casos de estudio}

En este trabajo se presentan dos edificios no residenciales localizados en diferentes continentes (América y Europa), con características particulares en su estructura, uso y clima circundante. El primero es un edificio nuevo localizado en Cambre, provincia de A Coruña, comunidad autónoma de Galicia, en el noroeste de España (latitud $43^{\circ} 18^{\prime} 22.55^{\prime \prime} N$, longitud $8^{\circ} 17^{\prime} 36.13^{\prime \prime} O$ ), a una altura de $99 \mathrm{~m}$ por encima del nivel del mar. El segundo es un edificio situado en San Nicolás de los Garza, municipio de Nuevo León, en el noreste de México (latitud $27^{\circ} 32^{\prime} 02^{\prime \prime} N$, longitud $99^{\circ} 58^{\prime} 33^{\prime \prime}$ E), a una altura de 239m sobre el nivel del mar.

\subsection{Condiciones climáticas}

De acuerdo con el mapa de Köppen-Geiger [91], basado en los valores mensuales y anuales de la temperatura media diaria del aire y las precipitaciones (Clasificación Climática Geiger), la ubicación española se clasifica en la región Cfb (C - templado, f - sin temporada seca, b - verano cálido), donde el clima oceánico tiene un verano suave con precipitaciones mínimas y una temperatura que no excede de $22^{\circ} \mathrm{C}$ en promedio en el mes más cálido y, donde al menos cuatro meses tienen un promedio de más de $10^{\circ} \mathrm{C}$. En esta clasificación no hay diferencia de precipitación significativa entre las estaciones.

Por parte del territorio mexicano se pueden identificar cuatro tipos principales de climas. Las condiciones áridas son predominantes en la región norte, el trópico seco en el oeste, las condiciones templadas se presentan en el centro y el trópico húmedo en la parte sureste. En el caso de México bajo esta misma clasificación, San Nicolás de los Garza puede ser considerada como BSh (BS - semiárido, h - caliente). Un clima semiárido o estepa (BSh) en una región se caracteriza por el hecho de que recibe una precipitación por debajo de la evapotranspiración potencial, aunque no 
extremadamente. Los climas semiáridos tienden a sustentar la vegetación corta o achaparrada, con áreas semiáridas generalmente dominadas por pastos o arbustos. Además, la temperatura media anual supera $\operatorname{los} 18^{\circ} \mathrm{C}$.

Los valores climáticos generales, como la temperatura del aire, la humedad relativa, la irradiancia directa (solar) y la irradiancia horizontal difusa, se toman de una base de datos por hora obtenida por la plataforma Equs [92] y de la base de datos Meteonorm [93] que se encuentra disponible en TRNSYS.

\subsection{Estructura y uso de los casos de estudio}

La base del primer edificio de forma rectangular tiene un área de $218.14 \mathrm{~m}^{2}$ (Fig. 3.1) distribuidos en dos plantas, definidas como planta baja y primer piso; el nivel inferior se utiliza como almacén (espacio sin climatizar) y el nivel superior correspondiente a una zona climatizada con oficinas, salas de reuniones, cocina y otros usos. Este edificio alberga la empresa Fridama Instalaciones, especializada en el diseño, implementación y mantenimiento de instalaciones de aire acondicionado, electricidad y control. El horario de trabajo se considera de lunes a viernes durante todo el año de las 08:30am a las 06:00pm. La pared principal tiene una dirección suroeste, donde se encuentra la entrada principal al almacén que da acceso a vehículos de carga, para el transporte de la mercancía que se comercializa en el inmueble.
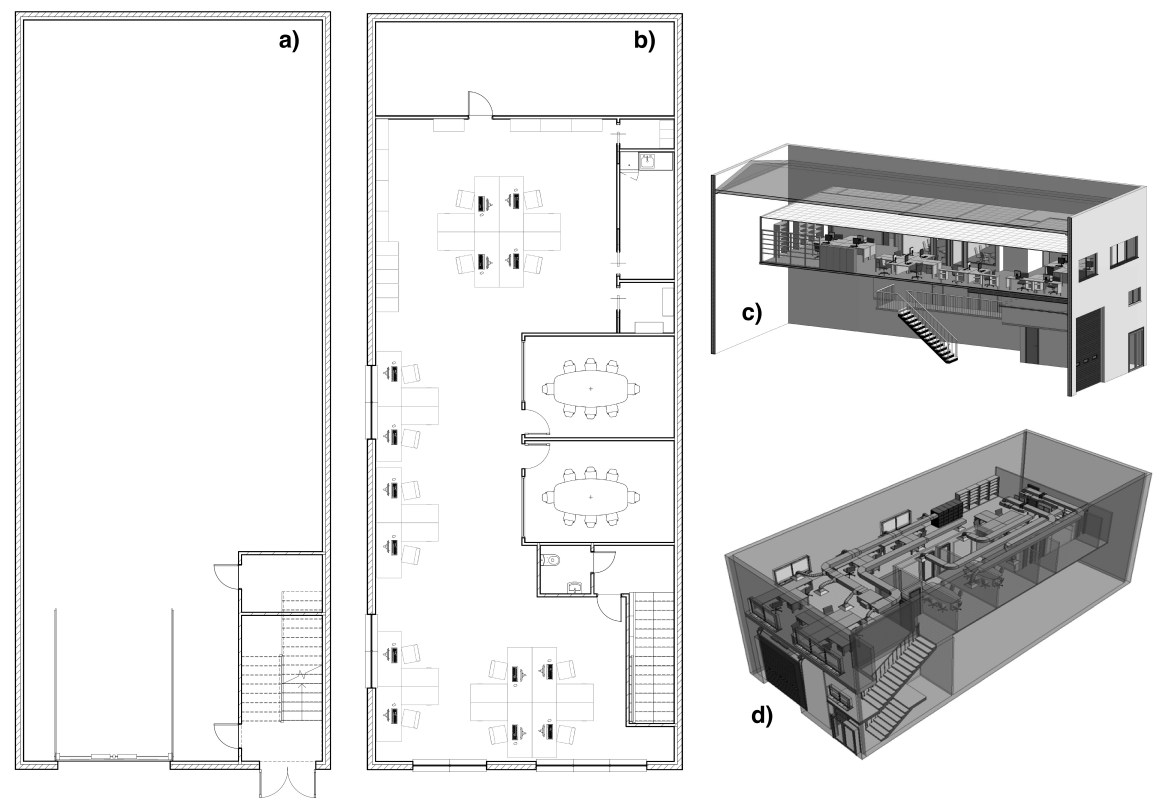

Figura 3.1: Planos arquitectónicos del primer caso de estudio: (a) planta baja; (b) primer piso. Geometría del modelo numérico: (c) vista noreste y (d) vista suroeste. 
Para el segundo caso de estudio, la base tiene forma de " $L$ ", cubriendo un área de $1214 m^{2}$ en cada una de sus tres alturas que definimos como planta baja (GF), primer piso (FF) y segundo piso (SF). La GF corresponde a un estacionamiento sin cerramientos verticales y un área de recepción, mientras que los otros dos pisos corresponden a aulas, salas de reuniones, laboratorios, oficinas, etc. (Fig. 3.2). La función principal del edificio es Centro de Investigación en Ciencias Físico Matemáticas (CICFIM) que se encuentra detrás de la Facultad de Ciencias Físico Matemáticas (FCFM) de la Universidad Autónoma de Nuevo León (UANL). Su acceso principal se encuentra en la fachada orientada al este, mientras su cara oeste colinda con las instalaciones del estadio José Ma. Chico. Este edificio inicia las actividades de lunes a viernes a las 07:00am y termina a las 09:00pm y el horario del día sábado es de 10:00am a 02:00pm. En la Fig. 3.3 podemos observar las vistas del edificio.
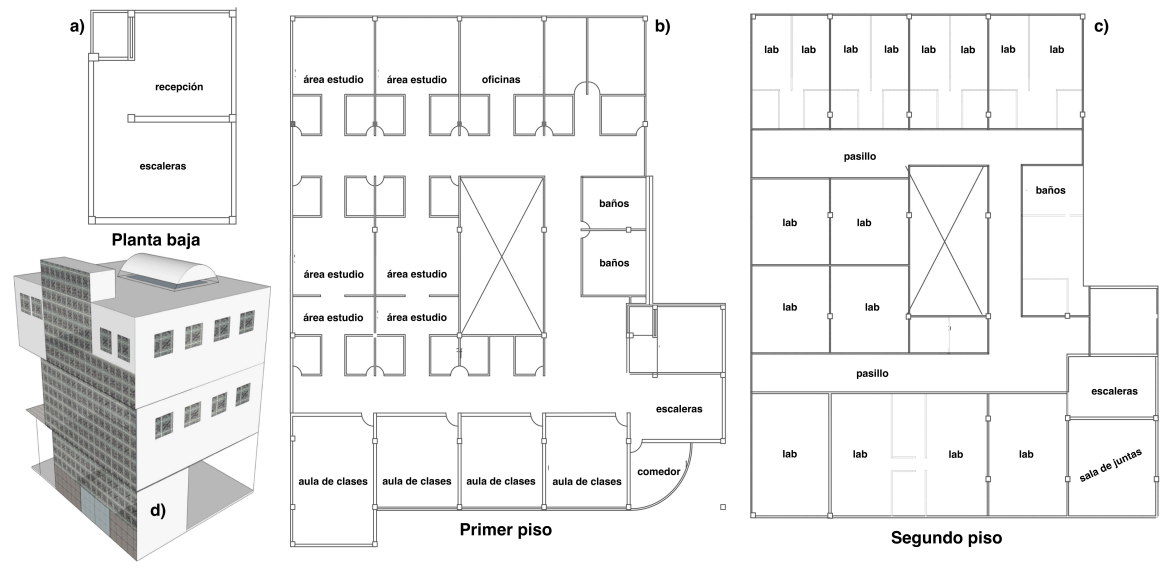

Figura 3.2: Planos arquitectónicos del segundo caso de estudio: (a) planta baja; (b) primer piso; (c) segundo piso. Geometría del modelo numérico: (d) vista noreste.

Para ambos casos de estudio, las características de las ventanas se describen en la Tabla 3.1. Se han definido 6 tipos de ventanas para el caso de México y 1 para el caso de España. Entre sus principales características se encuentran: porcentaje de marco, transmitancia térmica $(U)$ y la ganancia solar $(G-$ Value $)$, coeficiente utilizado para describir la cantidad de ganancia solar o radiación solar que entra en un espacio cerrado a través de un cristal o un material traslúcido. 


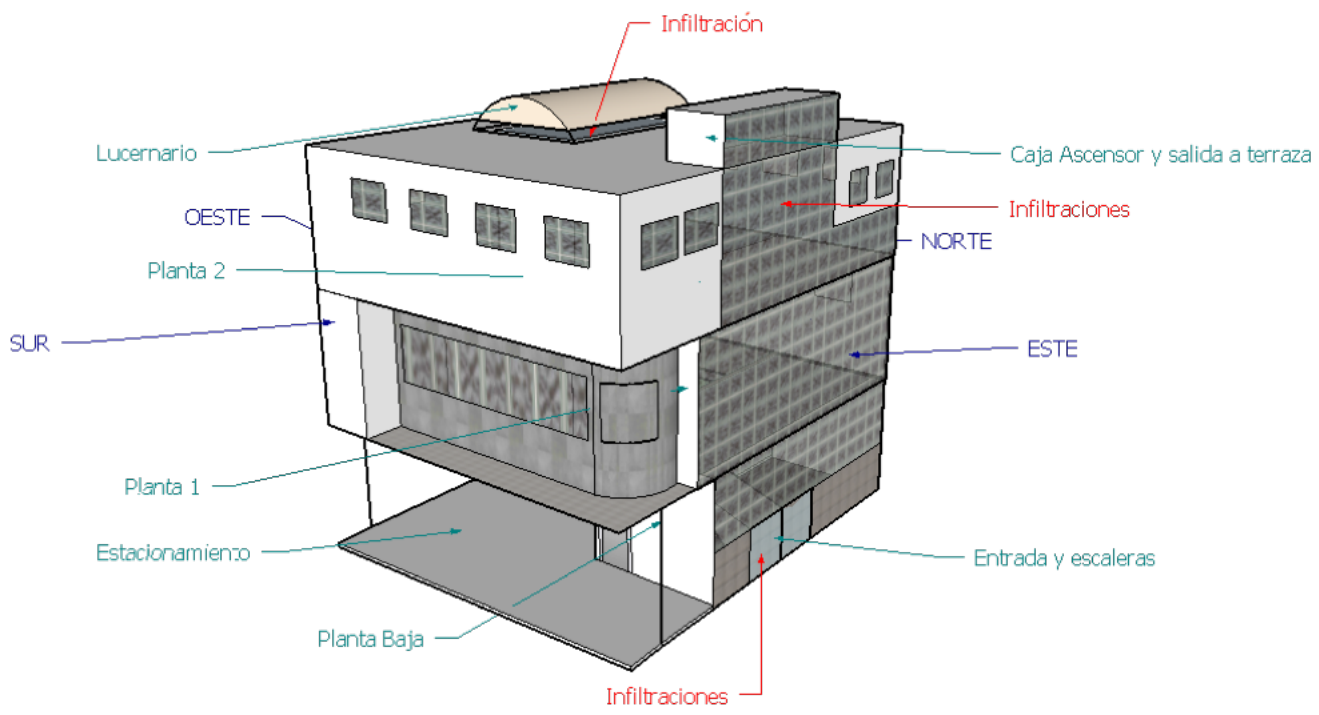

Figura 3.3: Geometría del modelo numérico en el software de diseño gráfico SketchUp®.

\begin{tabular}{|c|c|c|c|c|c|c|}
\hline \multirow{2}{*}{ Casos } & \multirow{2}{*}{ Tipos } & \multirow{2}{*}{ Tipos de vidrio } & Espesor & $\%$ & $U$ & $G-$ Value \\
\cline { 4 - 7 } & & $(\mathrm{mm})$ & Marco & $\left(\mathrm{W} / \mathrm{m}^{2} \mathrm{~K}\right)$ & $\left(\mathrm{kg} / \mathrm{m}^{3}\right)$ \\
\hline \hline \multirow{2}{*}{ 1: España } & Tipo A & Doble vidrio de baja emisividad & $4 / 6 / 4$ & 15 & 3.44 & 0.76 \\
\hline \hline \multirow{3}{*}{ 2: México } & & & & & \multirow{2}{*}{0.762} \\
\cline { 3 - 7 } & Tipo 1 & Simple con control solar & 6 & 5 & 5.73 & 0.482 \\
\cline { 2 - 7 } & Tipo 2 & Simple claro & 6 & 15 & 5.73 & 0.837 \\
\cline { 2 - 7 } & Tipo 3 & Doble claro & $6 / 12 / 6$ & 10 & 3.21 & 0.722 \\
\cline { 2 - 7 } & Tipo 5 & Simple claro & 6 & 35 & 5.73 & 0.837 \\
\cline { 2 - 7 } & Tipo 6 & Simple con control solar & 6 & 25 & 5.73 & 0.888 \\
\hline
\end{tabular}

Tabla 3.1: Propiedades térmicas y estructurales en ventanas.

En el primer edificio (España) hay dos tipos de zonas (WAC - sin calefactar y AC - calefactada ), dependiendo si hay aire acondicionado o no (ver Tabla 3.2). La segunda edificación (México) está distribuida en tres particiones (GF - FF - planta baja y planta $1, \mathrm{SF}$ - planta 2 y SL - lucernario), debido a los diferentes usos y temperaturas en el interior.

El edificio en España está construido con tres tipos de cerramientos: las paredes verticales, el piso y el techo. En la Tabla 3.3 mostramos los espesores de cada una 


\begin{tabular}{|c|c|c|c|}
\hline Casos & Zonas & Usos & Características físicas \\
\hline \multirow{2}{*}{ 1: España } & Sin calefactar(WAC) & Almacén y cámara de aire del techo & $\begin{array}{c}\text { Área }\left(253.36 \mathrm{~m}^{2}\right) \\
\text { Volumen }\left(1910 \mathrm{~m}^{3}\right) \\
\text { Capacitancia }\left(2292.71 \mathrm{~kJ} /{ }^{\circ} \mathrm{K}\right) .\end{array}$ \\
\hline & $\begin{array}{c}\text { Calefactada } \\
\text { (AC) }\end{array}$ & $\begin{array}{c}2 \text { oficinas, } 2 \text { salas de juntas, } \\
\text { almacén pequeño, cuarto de redes, } \\
\text { cocina, acceso y cuarto de limpieza }\end{array}$ & $\begin{array}{c}\text { Área }\left(218.13 \mathrm{~m}^{2}\right) \\
\text { Volumen }\left(610.78 \mathrm{~m}^{3}\right) \\
\text { Capacitancia }\left(732.94 \mathrm{~kJ} /{ }^{\circ} \mathrm{K}\right)\end{array}$ \\
\hline \multirow{3}{*}{ 2: México } & Zona GF-FF & $\begin{array}{c}\text { GF: recepción } \\
\text { FF: laboratorios, oficinas, } \\
\text { baños y áreas de estudio, comedor }\end{array}$ & $\begin{array}{c}\text { Área }\left(1142.68 \mathrm{~m}^{2}\right) \\
\text { Volumen }\left(3366.99 \mathrm{~m}^{3}\right) \\
\text { Capacitancia }\left(4040.39 \mathrm{~kJ} /{ }^{\circ} \mathrm{K}\right)\end{array}$ \\
\hline & Zona SF & $\begin{array}{c}\text { Laboratorios, oficinas, } \\
\text { baños, centro de cómputo, } \\
\text { cuarto de limpieza y sala de juntas }\end{array}$ & $\begin{array}{c}\text { Área }\left(1142.68 \mathrm{~m}^{2}\right) \\
\text { Volumen }\left(3225.0 \mathrm{~m}^{3}\right) \\
\text { Capacitancia }\left(3870.0 \mathrm{~kJ} /{ }^{\circ} \mathrm{K}\right)\end{array}$ \\
\hline & Zona SL & Espacio abierto sin utilizar & $\begin{array}{c}\text { Área }\left(84.0 \mathrm{~m}^{2}\right) \\
\text { Volumen }\left(462.0 \mathrm{~m}^{3}\right) \\
\text { Capacitancia }\left(554.4 \mathrm{~kJ} /{ }^{\circ} \mathrm{K}\right)\end{array}$ \\
\hline
\end{tabular}

Tabla 3.2: Distribución de los casos de estudio.

de las capas de los cerramientos, donde sus espesores totales respectivos (t) son $46.5 \mathrm{~cm}, 30.4 \mathrm{~cm}$ y $185 \mathrm{~cm}$. El edificio en México (Tabla 3.3) tiene cuatro tipos de cerramientos: paredes internas $(9 \mathrm{~cm})$ y externas $(35 \mathrm{~cm})$, así como divisiones de $10 \mathrm{~cm}$ de espesor. En este caso, los mismos materiales se usaron en el piso y el techo. En esta misma tabla se muestran las características físicas de cada capa de material como: conductividad térmica $(\kappa)$, capacidad calorífica $\left(C_{p}\right)$ densidad $(\rho)$ y resistencia $(R)$ en casos específicos. 


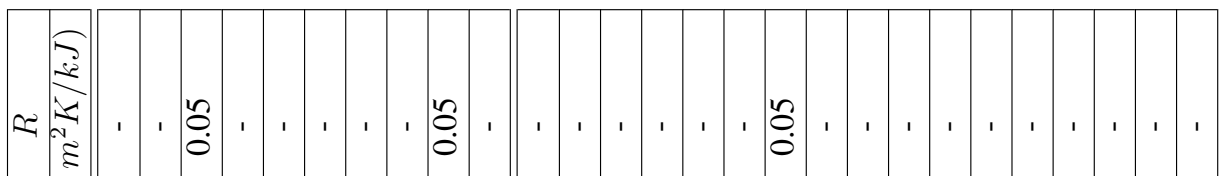
$\approx$

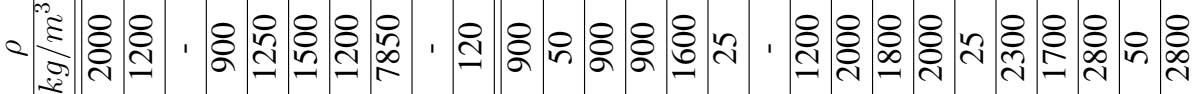

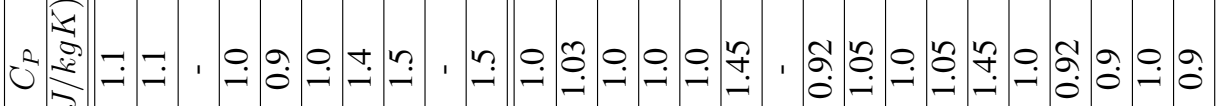

$\leq$

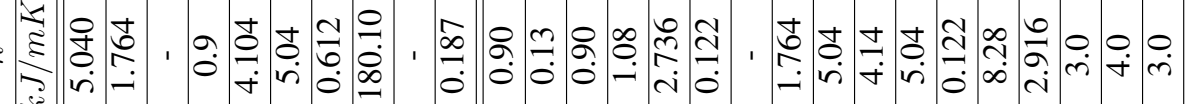
$\therefore$

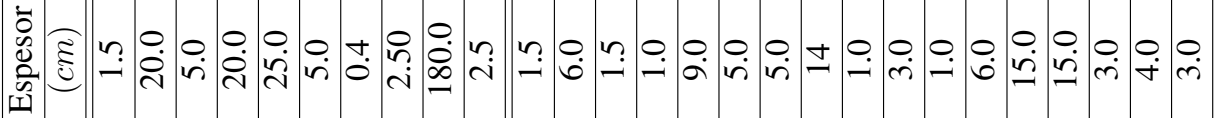

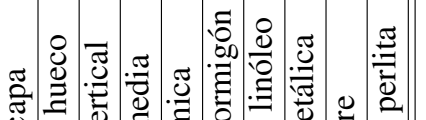

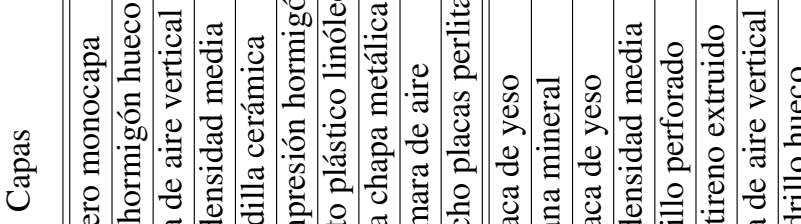

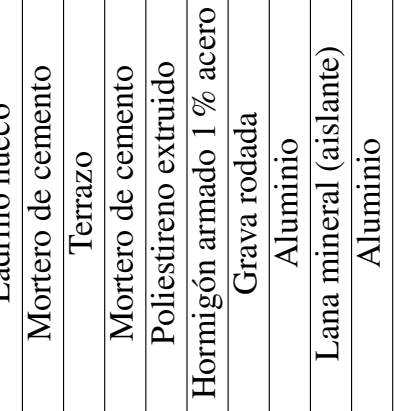

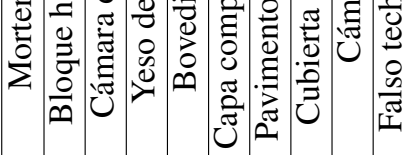

离 疍

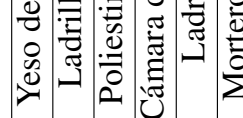

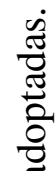

水

$\frac{0}{0}$

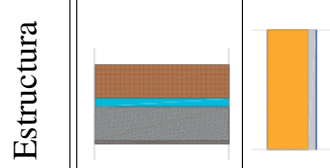
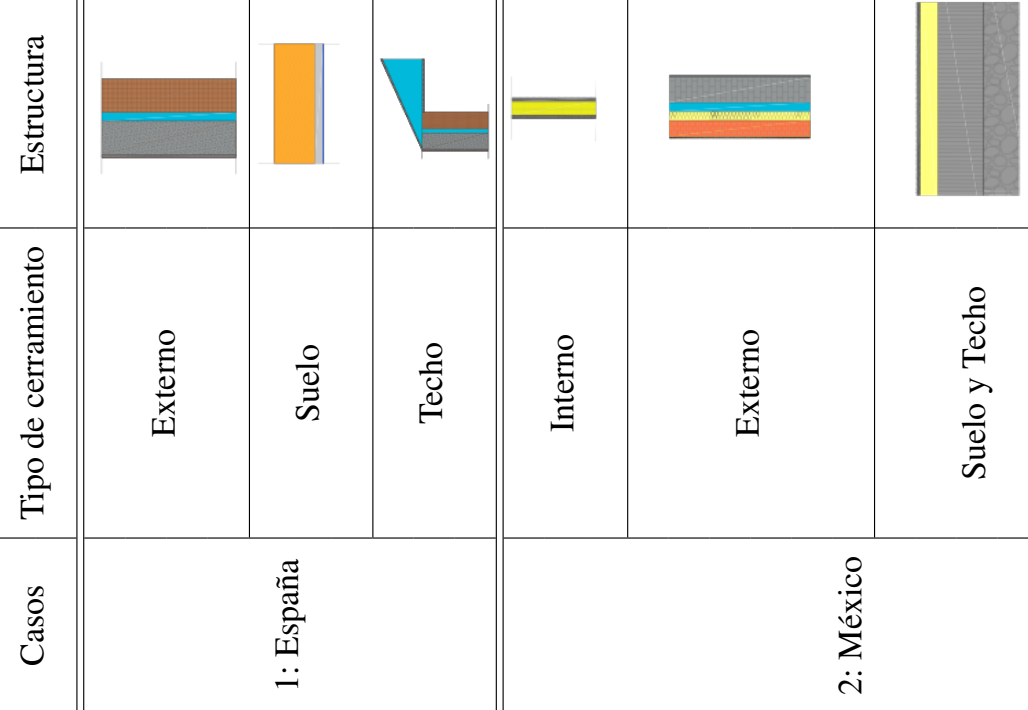

$\frac{O}{0}$

$\frac{2}{2}$

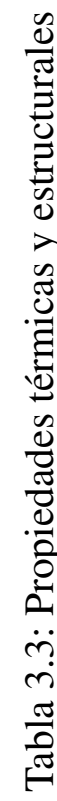




\section{Capítulo 4}

\section{Metodología}

El objetivo principal de este trabajo es mostrar las consecuencias, con base en los resultados de las simulaciones basadas en BIM, de las decisiones subjetivas tomadas por los diseñadores al utilizar el estándar de ergonomía ambiental ISO 7730, para calcular los rangos de confort térmico y su impacto en la demanda de energía de los edificios, así como su repercusión económica. La sensación térmica experimentada por un ser humano se debe principalmente al equilibrio térmico general del cuerpo, que depende de la actividad física (met) y vestimenta (clo) del sujeto, así como de los parámetros ambientales: temperatura del aire, temperatura radiante, velocidad y humedad del aire. Debemos enfatizar que la definición de los parámetros de la indumentaria humana y la actividad la realiza subjetivamente el diseñador y, por lo tanto, nos referimos a estas cantidades como Parámetros Humanos Subjetivos (SHPs).

Los índices $P M V$ (Predicted Mean Vote) y PPD (Predicted Percentage Dissastisfied) se usan comúnmente para expresar una incomodidad cálida y fría para el cuerpo como un todo, aunque la insatisfacción térmica también puede ser causada por el calentamiento o enfriamiento no deseado de una parte particular del cuerpo (incomodidad local). Por lo tanto, cuando se conocen los factores previos, se puede predecir la sensación térmica de los ocupantes, usando una escala de siete puntos que varía desde frío (-3) hasta caliente (3), siendo 0 el valor de "sensación neutra" [43]. El $P M V$ se puede calcular a partir de tablas o se puede medir directamente con un instrumento [94]. La metodología propuesta en este trabajo consiste en obtener primero los rendimientos térmicos de los edificios y, luego, realizar el análisis de sensibilidad para los parámetros humanos subjetivos (SHPs) utilizados en el cálculo del confort térmico, con el propósito de identificar cuál de ellos tiene mayor influencia en las salidas de simulación basadas en BIM. 


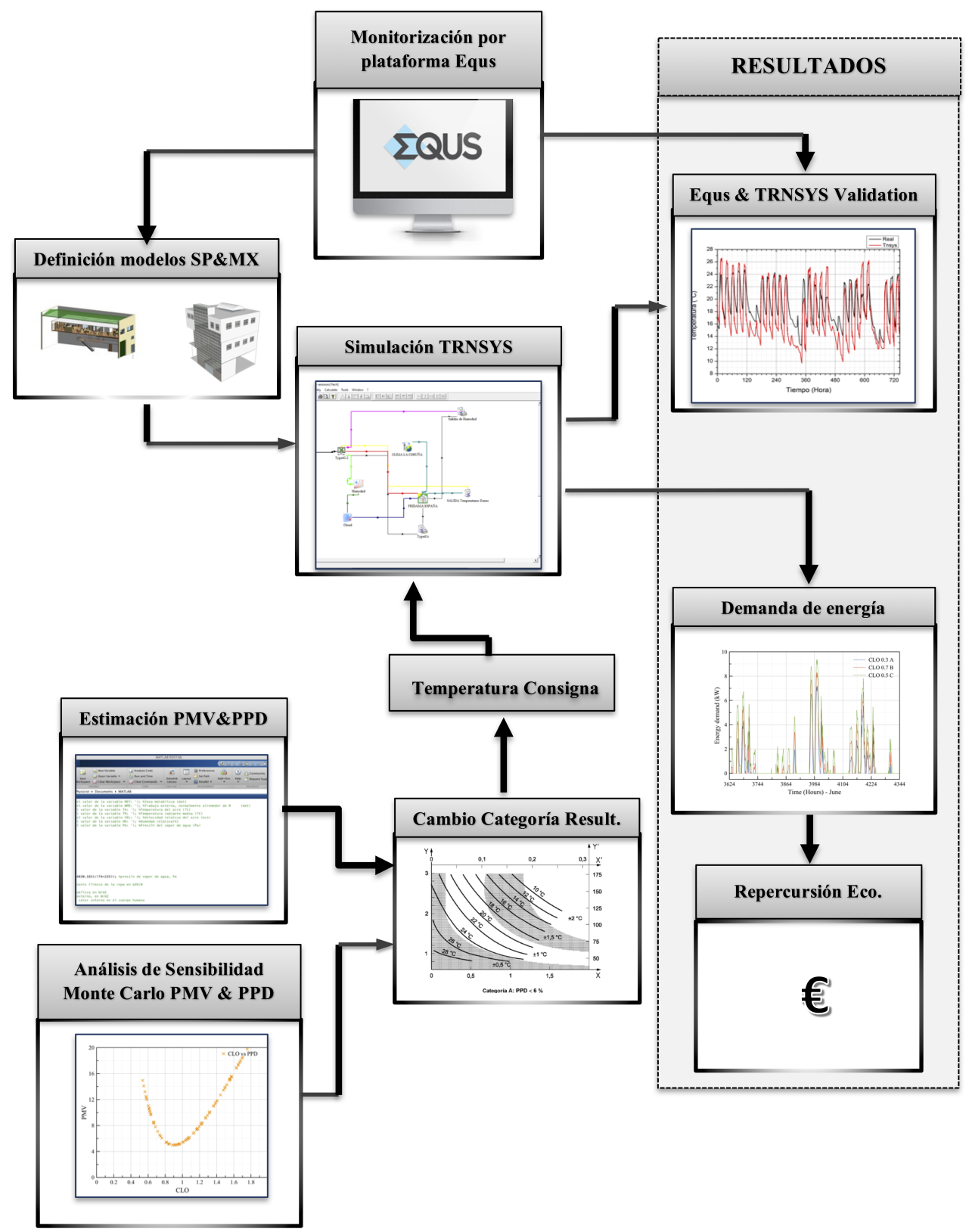

Figura 4.1: Esquema de la metodología utilizada.

La Fig. 4.1 muestra un esquema del procedimiento aplicado para ambos casos, que consta de los siguientes pasos y que se detallaran en las secciones posteriores:

1. Seguimiento en tiempo real de las variables principales utilizando la plataforma web Equs. 
2. Modelado TRNSYS, obteniendo resultados tales como temperatura y demanda de energía.

3. Estimación matemática de los índices de $P M V$ y $P P D$.

4. Análisis de sensibilidad (Monte Carlo) para determinar qué valores de los parámetros (factor de actividad y factor de vestimenta) producen una variación significativa en las salidas de $P M V$ y $P P D$.

5. Se establecen nuevas temperaturas de punto de ajuste, dependiendo de los cambios en las categorías térmicas (detalladas en la sección 4.5)en el paso anterior.

6. Verificación de la simulación térmica con datos reales de la base de datos Equs.

7. Recálculo de la demanda de energía y del impacto económico, con base en los dos pasos anteriores.

\subsection{Monitorización por plataforma web: Equs}

En esta sección se utiliza una plataforma web para la gestión energética de edificios llamada Equs, desarrollada con el objetivo de medir, transmitir y manejar los consumos de energía y las variables ambientales como la temperatura, la humedad y los niveles de $\mathrm{CO}_{2}$. Además, esta herramienta tiene la posibilidad de controlar desde cualquier lugar, de forma remota, mediante el sistema SCADA (Supervisor de Control y Adquisición de Datos), asegurando la calidad de los resultados estadísticos (Fig. 4.2).

Equs recopila en su base de datos la información enviada por los diferentes sensores, lo que nos permite conocer la variación de las variables físicas de forma remota, ya que el acceso a estos datos se puede obtener desde cualquier computadora u otros dispositivos como teléfonos inteligentes conectados a internet, por lo que es muy simple el acceso a los datos, lo que se puede hacer en el mismo dispositivo accediendo a los valores, en tiempo real [92]. 

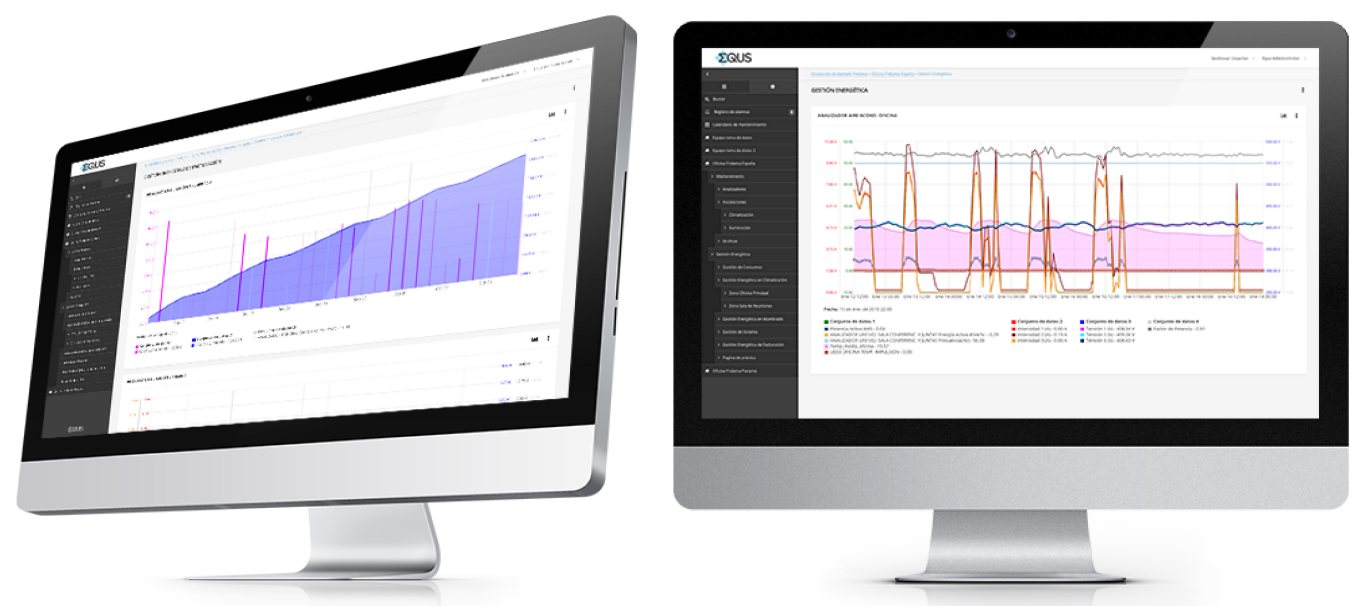

Figura 4.2: Captura de pantalla de la plataforma web "Equs" (http://equs-app.com).

\subsection{Modelado de los edificios: TRNSYS}

A través de la herramienta de simulación dinámica TRNSYS, podemos definir y evaluar con precisión el confort y la demanda de energía de los edificios; en ambos casos, trabajamos con modelos de edificios de múltiples zonas. TRNSYS se compone de dos partes: la primera es un motor que lee y procesa el archivo de entrada, resuelve el sistema (modelo y sus componentes) de manera iterativa, determina la convergencia y traza las variables del sistema; la segunda parte es una biblioteca de componentes, cada uno de los cuales modela el rendimiento de una parte de todo el sistema.

Un enfoque interesante de TRNSYS es convertir los problemas complejos, como son los modelos de edificios de múltiples zonas, en componentes más pequeños, con el fin de permitir que el diseñador establezca las conexiones entre el edificio y muchos otros subsistemas/componentes en el entorno de simulación [95].

En lo que sigue, se evaluarán las demandas de energía de los edificios después de realizar el análisis de sensibilidad de $P M V$ y $P P D$ respecto $c l o$ y met. Para ello se optó por utilizar el programa TRNSYS. Para llevar a cabo la simulación del edificio, tanto en España como en México, se han introducido en el programa todos los insumos, dimensiones, ubicación, características de construcción, etc., necesarios. La simulación TRNSYS para diferentes valores del factor de vestimenta y factor de actividad (a las condiciones de temperatura interior establecidas por la normativa ISO 7730 [44]) nos proporcionará diferentes valores de la demanda energética que permitirá cuantificar el impacto de la variación en cada uno de los parámetros analizados en el estudio de sensibilidad. 


\subsubsection{Definición de los modelos en programa de simulación: $T R N$ - Build}

La introducción de los edificios en el software TRNSYS se realizó de la siguiente manera: el primer paso fue la implementación de las zonas y todos sus elementos como ubicación, orientaciones, muros, capas de materiales, ganancias, ventilación, infiltración, horarios y confort en TRNBuild. El segundo paso corresponde a incluir en los modelos TRNSYS datos como clima, entradas como el edificio descrito anteriormente, para obtener salidas que mostrarán a través de gráficos el rendimiento térmico y las demandas de energía de los edificios. Para ello, obtuvimos la base de datos de los edificios que incluye información como orientaciones de las fachadas, materiales para las paredes y ventanas, la demanda de energía de los equipos instalados, las horas de trabajo, la ocupación, etc. El proceso de definición de los edificios en TRNBuild, incluye la siguiente información:

\section{- Estructura del edificio}

Los materiales detallados en las Tablas 3.1 y 3.3 se asignaron desde la base de datos del software.

\section{- Ubicación y orientaciones}

Esta información fue descrita en la sección 3.1.

\section{- Definición de zonas}

Las zonas descritas en la Tabla 3.2, se crearon como WAC y AC para el primer caso, GF-FF, SF y SL para el segundo caso en TRNBuild Manager.

\section{- Horarios}

En la Tabla 4.1 se muestran los diferentes tipos de horarios creados para cada edificio; establecemos un valor de 1 en los períodos donde hay actividad en el edificio, en cualquier otro caso, incluido el fin de semana, el valor es 0. 


\begin{tabular}{|c|c|c|c|c|}
\hline Casos & \multicolumn{2}{|c|}{ Tipos de horarios } & Horas & Uso \\
\hline \multirow{3}{*}{ 1: España } & \multirow{3}{*}{ Diario } & Diario 1 & $08: 00$ a $18: 30$ & 1 \\
\cline { 3 - 5 } & & Diario 2 & $13: 00$ a $16: 00$ & 1 \\
\cline { 3 - 5 } & & Diario 3 & $08: 00$ a 13:00 & 1 \\
\cline { 3 - 5 } & \multirow{3}{*}{ Semanal } & Semanal 1 & Lunes a Viernes & Diario 1 \\
\cline { 3 - 5 } & & Semanal 2 & Lunes a Viernes & Diario 2 \\
\cline { 3 - 5 } & & Semanal 3 & Lunes a Viernes & Diario 3 \\
\hline \hline \multirow{3}{*}{ 2: México } & \multirow{2}{*}{ Diario } & Diario A & 09:00 a 21:00 & 1 \\
\cline { 3 - 5 } & & Diario B & $07: 00$ a 21:00 & 1 \\
\cline { 3 - 5 } & & Diario C & $10: 00$ a 14:00 & 1 \\
\cline { 3 - 5 } & \multirow{2}{*}{ Semanal } & Semanal A & Lunes a Viernes & Diario A \\
& & & Sabado & Diario C \\
\cline { 3 - 5 } & & Semanal B & Lunes a Domingo & Diario B \\
\hline
\end{tabular}

Tabla 4.1: Tipos de horarios en los edificios.

\section{- Infiltraciones}

La Tabla 4.2 resume las infiltraciones para el verano y el invierno en ambos casos de estudio. Esta información se calculó en base a la normativa ASHRAE 55.

\begin{tabular}{|c|c|c|c|c|}
\hline \multirow{3}{*}{ Casos } & \multirow{3}{*}{ Horas } & & ciones & \multirow{3}{*}{$\begin{array}{c}\text { Ventilación } \\
\text { Cambio de aire } \\
\text { por hora }(\text { renov } / h)\end{array}$} \\
\hline & & \multicolumn{2}{|c|}{ Cambio de aire por hora $($ renov $/ h)$} & \\
\hline & & Verano & Invierno & \\
\hline \multirow{2}{*}{ 1: España } & WAC & 0.143 & 0.031 & 5.0 \\
\hline & $\mathrm{AC}$ & 0.066 & 0.067 & 1.65 \\
\hline \multirow{3}{*}{ 2: México } & GF-FF & 3.39 & 1.80 & 8.0 \\
\hline & $\mathrm{SF}$ & 3.41 & 1.81 & 8.0 \\
\hline & SL & 25.27 & 13.41 & 10.0 \\
\hline
\end{tabular}

Tabla 4.2: Infiltraciones y ventilación para cada zona en verano e invierno.

\section{- Ventilación}

Existen diferentes métodos para realizar el cálculo de la ventilación mínima requerida en edificios, que dependen del uso o nivel de estanqueidad ${ }^{1}$; para 
calcular la ventilación utilizamos la norma EN 13779, ASHRAE 62 R. Se estimó un valor para cada zona que se muestra en la Tabla 4.2.

\section{- Ganancias internas}

Las ganancias internas de los edificios son una de las propiedades más importantes para introducir en el análisis del comportamiento térmico. Esta información se muestra en las Tablas 4.3 y 4.4 .

\begin{tabular}{|c|c|c|c|c|}
\hline Casos & Ganancias & Descripción & Horario & Energía total (W) \\
\hline \multirow{10}{*}{ 1: España } & Ocupación & $\begin{array}{l}15 \text { personas adultas con } \\
\text { actividad de oficina }\end{array}$ & Semanal 1 & 2240 \\
\hline & Computadoras & $\begin{array}{c}16 \mathrm{PCs} \\
\text { con monitores }\end{array}$ & Semanal 1 & 2240 \\
\hline & Luz artificial & $\begin{array}{c}37 \text { lámparas fluorescentes } \\
\text { en } 218.12 \mathrm{~m}^{2}\end{array}$ & Semanal 1 & 3600 \\
\hline & \multirow{7}{*}{ Otras ganancias } & Equipo de aire acondicionado & Semanal 1 & 3332 \\
\hline & & Cafetera (10 tazas) & Semanal 2 & 1500 \\
\hline & & Copiadora tipo oficina & Semanal 3 & 1060 \\
\hline & & Microondas & Semanal 2 & 600 \\
\hline & & Refrigerador & Encedido & 322 \\
\hline & & Plotter & Semanal 1 & 250 \\
\hline & & TV & Semanal 1 & 90 \\
\hline
\end{tabular}

Tabla 4.3: Datos de entrada de ganancias internas en la zona de AC en el caso de España.

\footnotetext{
${ }^{1}$ El Código Técnico de la Edificación (CTE), aprobado por Real Decreto en 2006, define qué es la permeabilidad al aire.
} Según el documento, es la "propiedad de una ventana o puerta de dejar pasar el aire cuando se encuentra sometida a una presión diferencial. La permeabilidad al aire se caracteriza por la capacidad de paso del aire, expresada en $m^{3} / h$, en función de la diferencia de presiones". La estanqueidad es una propiedad que se basa en la capacidad para evitar que entren partículas externas al interior de una pieza, circuito o habitáculo, ya sea agua, aire, polvo, etc. 


\begin{tabular}{|c|c|c|c|c|c|c|c|}
\hline \multirow{2}{*}{ Casos } & \multirow[t]{2}{*}{ Ganancias } & \multicolumn{2}{|c|}{ Zonas } & \multirow{2}{*}{ Descripción } & \multirow{2}{*}{ Horario } & \multicolumn{2}{|c|}{ Energía total (W) } \\
\hline & & GF-FF & SF & & & GF-FF & SF \\
\hline \multirow{15}{*}{ 2: México } & Ocupación & 63 & 51 & $\begin{array}{l}15 \text { personas adultas con } \\
\text { actividad de oficina }\end{array}$ & Semanal A & 9450 & 7650 \\
\hline & Ordenadores & 54 & 50 & $\begin{array}{c}16 \mathrm{PCs} \\
\text { con monitores }\end{array}$ & Semanal A & 7560 & 7000 \\
\hline & \multirow{6}{*}{ Iluminación } & \multirow{2}{*}{36} & \multirow{2}{*}{9} & \multirow{2}{*}{$\begin{array}{c}\text { Lámparas } \\
\text { fluorescentes }(64 W)\end{array}$} & Semanal A & 2304 & 576 \\
\hline & & & & & & & \\
\hline & & \multirow{2}{*}{60} & \multirow{2}{*}{46} & \multirow{2}{*}{$\begin{array}{c}\text { Lámparas } \\
\text { fluorescentes }(80 \mathrm{~W})\end{array}$} & Semanal A & 4800 & 3680 \\
\hline & & & & & & & \\
\hline & & \multirow{2}{*}{64} & \multirow{2}{*}{62} & \multirow{2}{*}{$\begin{array}{c}\text { Lámparas } \\
\text { fluorescentes }(40 \mathrm{~W})\end{array}$} & Semanal A & 2560 & 2480 \\
\hline & & & & & & & \\
\hline & \multirow{7}{*}{ Otras ganancias } & - & - & \multicolumn{2}{|c|}{ Equipo de aire acondicionado } & \multicolumn{2}{|c|}{21320} \\
\hline & & 5 & 3 & \multicolumn{2}{|c|}{ Cafetera (10 tazas) } & \multicolumn{2}{|c|}{1500} \\
\hline & & 2 & 1 & \multicolumn{2}{|c|}{ Copiadora tipo oficina } & \multicolumn{2}{|c|}{700} \\
\hline & & 1 & - & \multicolumn{2}{|l|}{ Microondas } & \multicolumn{2}{|c|}{600} \\
\hline & & 2 & 1 & \multicolumn{2}{|l|}{ Refrigerador } & \multicolumn{2}{|c|}{322} \\
\hline & & 10 & 12 & \multicolumn{2}{|l|}{ Plotter } & \multicolumn{2}{|c|}{250} \\
\hline & & 2 & - & \multicolumn{2}{|l|}{ TV } & \multicolumn{2}{|c|}{90} \\
\hline
\end{tabular}

Tabla 4.4: Datos de entrada de ganancias internas en el caso de México.

\section{- Confort}

Las sensaciones térmicas son diferentes entre las personas, incluso en el mismo entorno. A pesar de que los sensores producen los mismos resultados independientemente de la posición geográfica en la que se toma una medición, éste no es el caso para las personas. De hecho, las personas que se alojan en espacios muy similares, sujetos al mismo clima y pertenecientes a una cultura común, emiten opiniones muy diferentes sobre el confort térmico debido a la combinación de un gran número de factores que afectan a la percepción de los seres humanos.

\begin{tabular}{|c|c|c|}
\hline Ganancias & Descripción & Valor \\
\hline \hline \multirow{2}{*}{ Factor de vestimenta } & $\begin{array}{c}\text { Invierno: ropa interior, camisa, pantalones, } \\
\text { chaqueta térmica, calcetines, zapatos }\end{array}$ & $1.2(\mathrm{clo}) / 0.185\left(\mathrm{~m}^{2} \cdot \mathrm{K} / \mathrm{W}\right)$ \\
\cline { 2 - 3 } & $\begin{array}{c}\text { Verano: ropa interior, camisa, } \\
\text { pantalones, calcetines, zapatos }\end{array}$ & $0.70(\mathrm{clo}) / 0.110\left(\mathrm{~m}^{2} \cdot \mathrm{K} / \mathrm{W}\right)$ \\
\hline \hline Factor metabólico & $\begin{array}{c}\text { Trabajo sentado y liviano } \\
\text { (oficina, hogar, escuela, laboratorio) }\end{array}$ & $1.2(\mathrm{met}) / 70\left(\mathrm{~W} / \mathrm{m}^{2}\right)$ \\
\hline \hline Trabajo externo & $\begin{array}{c}\text { En general, el trabajo externo } \\
\text { es alrededor de 0 }\end{array}$ & $0(\mathrm{met})$ \\
\hline \hline Velocidad de aire relativo & $\begin{array}{c}\text { La velocidad del aire en relación } \\
\text { con la persona }\end{array}$ & $0.4(\mathrm{~m} / \mathrm{s})$ \\
\hline
\end{tabular}

Tabla 4.5: Datos de entrada de confort en los dos edificios. 
El confort térmico es uno de los principales factores relacionado directamente con el comportamiento térmico del edificio; por esta razón, los valores de factor de vestimenta, tasa metabólica, velocidad relativa del aire se introdujeron en TRNSYS (basados en la normativa ISO 7730) [44]. La Tabla 4.5 muestra los valores para ambos casos, verano e invierno; decidimos establecer los mismos valores para la ropa y la actividad, ya que las características de los ocupantes son muy similares (debemos enfatizar que estos valores son decisiones subjetivas del diseñador). El análisis del impacto de estas decisiones es uno de los principales objetivos del presente trabajo

\subsubsection{Simulación de los modelos en TRNSYS Studio}

En esta sección, se presenta el modelo de simulación térmica dinámica en TRNSYS Studio del edificio descrito anteriormente en TRNBuild; para este propósito utilizamos el componente "Type56" de construcción multizona, que se puede conectar a una gran cantidad de otros componentes, incluidos los datos meteorológicos, sistemas HVAC, programas de ocupación, controladores, funciones de salida, almacenamiento de energía térmica, sistemas de energía renovable (solar), etc. Otros componentes utilizados fueron Type109-TMY2, Type62 y Type65.

El componente Type56 modela el comportamiento térmico de un edificio que tiene hasta 25 zonas térmicas este componente lee la descripción del edificio de un conjunto de archivos externos que tienen las extensiones *.bui, *.bld y *.trn. Los archivos se pueden generar en función de la información suministrada por el usuario ejecutando el programa del procesador llamado TRNBuild. Esta instancia de Type56 genera su propio conjunto de archivos de salida de resumen mensuales y por hora.

El Type109-TMY2 tiene como propósito general leer datos meteorológicos a intervalos de tiempo regulares de un archivo de datos,convertirlo en un sistema de unidades deseado y procesar los datos de radiación solar para obtener radiación de superficie inclinada y angulo de incidencia para un número arbitrario de superficies. El Type109 lee un archivo de datos meteorológico en el formato estandar TMY2, este formato es utilizado por la Base Nacional de Datos de Radiación Solar (EE.UU.), pero los archivos TMY2 se pueden generar desde muchos programas, como Meteonorm.

Mediante el Type62 TRNSYS implementa un enlace con Excel. La rutina de Fortran se comunica con Excel a través de un interfaz del Modelo de Objetos Componentes $(\mathrm{COM})$ para una rápida transferencia de datos. TRNSYS envía las entradas del componentes a las celdas dadas en sus hojas de trabajo en Excel.

El Type65 es un componente de gráficos en linea, se utiliza para mostrar las 
variables del sistema seleccionadas mientras la simulación está en progreso. Este componente es altamente recomendado y ampliamente utilizado, ya que proporciona información variable valiosa y permite a los usuarios ver inmediatamente si el sistema no está funcionando como se desea. Las variables seleccionadas se mostrarán en una ventana de trazado separada en la pantalla. En esta instancia los datos enviados al Type65 se imprimen automáticamente, una vez por vez, en un archivo externo definido por el usuario.

\subsection{Estimación del $P M V$ y $P P D$}

De acuerdo con la norma ISO 7730 [44], la evaluación de los niveles de confort

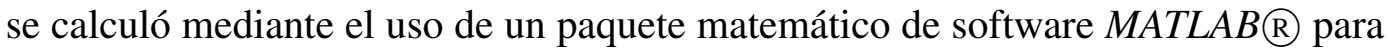
el estudio numérico interactivo de sistemas dinámicos (Fig. 4.3) [44]. En la sección de anexos se muestra a detalle dos diferentes maneras de realizar el cálculo de estos indices númericos.

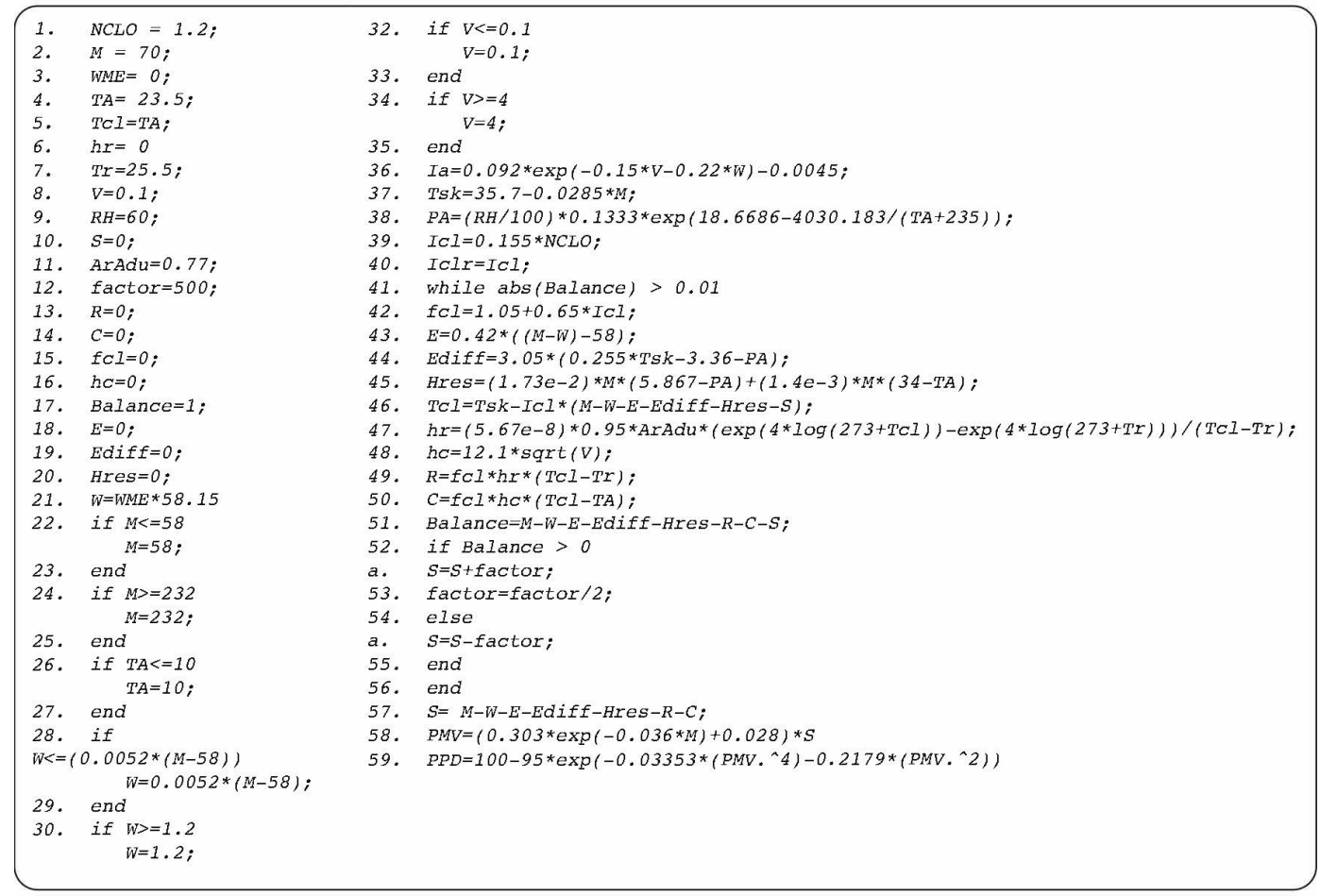

Figura 4.3: Estimación computacional de $P M V$ y $P P D$ basada en ISO 7730 [44].

El cambio de $P M V$ y $P P D$ en función de sus parámetros, como la tasa metabólica, el aislamiento de la ropa y la temperatura del aire, es más fácil de visualizar 
en MATLAB que en TRNSYS ya que se puede modificar sus parámetros de forma independiente y realizar estudios más específicos de ese tipo.

\subsection{Análisis de sensibilidad mediante el método Mon- te Carlo}

El objetivo de un análisis de sensibilidad (SA) en este trabajo es estudiar la respuesta del modelo simulado por TRNSYS con respecto a la variación de los parámetros de diseño específicos, como la vestimenta y la actividad.

El análisis de sensibilidad es ampliamente aplicable para relacionar los parámetros de entrada con el rendimiento general del edificio (por ejemplo, la temperatura o la demanda de calefacción o refrigeración) [85]. La contribución de cada entrada puede ser analizada por SA para preparar para la futura optimización de la energía, el medio ambiente y el rendimiento económico [96,97].

Los métodos basados en muestreo para la incertidumbre y el análisis de sensibilidad se han vuelto muy populares. El método Monte Carlo basado en muestreo es una técnica que opera un modelo varias veces con muestras aleatorias generadas a partir de distribuciones de entrada. Proporciona soluciones aproximadas a los análisis de incertidumbre y sensibilidad mediante la realización de experimentos de modelado estadístico $[98,99]$. El método puede manejar modelos de caja negra complejos, independientemente de la linealidad y la continuidad, y generar una distribución de probabilidad para cada salida dependiendo de los tipos de distribución de entrada [83].

En general, para un análisis de esta clase, consideremos una función $y(x)$ donde:

$$
y(x)=\left[y_{1}(x), y_{2}(x), \ldots, y_{n Y}(x)\right]
$$

$\mathrm{y}$

$$
x=\left[x_{1}, x_{2}, \ldots, x_{n X}\right]
$$

definen las salidas y entradas del análisis, respectivamente (ecuaciones 4.1 y 4.2). Sucesivamente, la variación en $x$ da como resultado una sensibilidad correspondiente en $y(x)$. Además, las distribuciones

$$
D=\left[D_{1}, D_{2}, \ldots, D_{n X}\right]
$$


necesitan estar bien definidas para caracterizar la incertidumbre asociada con los elementos de $x$, donde $D_{i}$ es la distribución asociada con $x_{i}$ para $i=1,2, \ldots, n X$.

Los pasos más comunes para aplicar el análisis de sensibilidad en el análisis del comportamiento energético del edificio son: determinar las variaciones de entrada, crear modelos de energía de edificios, ejecutar modelos de energía, recoger resultados de simulación, ejecutar análisis de sensibilidad y presentación de los resultados del análisis de sensibilidad [85]. Determinar las distribuciones de probabilidad de los parámetros de entrada son el primer paso en el análisis de sensibilidad. En el presente estudio, se utiliza el análisis de sensibilidad por el método de Monte Carlo, considerando $O_{i}$ objetivos que depende de $P_{j}$ parámetros (parámetros de entrada o aquellos que definen el sistema):

$$
O_{i}\left(P_{1}, P_{2}, \ldots, P_{N}\right) \quad i=1,2,3, . ., M
$$

\subsubsection{Análisis de sensibilidad mediante el uso de distribución uniforme}

Supongamos que el parámetro $P_{j}$ puede cambiar con un ancho $\Delta P_{j}$. Para analizar la influencia del parámetro $P_{j}$ en el objetivo $O_{i}$, procedemos primero generando un número aleatorio distribuido uniformemente $r \in[0,1]$. Donde una distribución uniforme es el modelo continuo más simple. Corresponde al caso de una variable aleatoria que sólo puede tomar valores comprendidos entre dos extremos a y b, de manera que todos los intervalos de una misma longitud (dentro de $(a, b)$ ) tienen la misma probabilidad [100].

De la anterior definición se desprende que la función de densidad debe tomar el mismo valor para todos los puntos dentro del intervalo $(a, b)$ (y cero fuera del intervalo). Es decir,

$$
f_{X}(\chi)=\left\{\frac{1}{b-a} \text { si } \chi \in(a, b) \text { y } 0 \text { si } \chi \notin(a, b)\right\}
$$

Ahora procedemos a generar un número aleatorio en base a la siguiente ecuación,

$$
P_{j}^{1}=\left(P_{j}-\Delta P_{j}\right)+2 r \cdot \Delta P_{j}, \quad P_{j}^{1} \in\left[P_{j}-\Delta P_{j}, P_{j}+\Delta P_{j}\right]
$$

Obteniendo el primer valor aleatorio $P_{j}^{1}$, con el valor de $N$ parámetros aleatorios generados $P_{j}^{1}(j=1,2,3, \ldots, N)$, obtenemos un valor de $O_{i}^{1}$. Esto es, podemos cambiar aleatoriamente todos los parámetros, en nuestro estudio variaremos aleatoriamente uno de ellos fijando el resto constante. 


\subsubsection{Análisis de sensibilidad mediante el uso de distribución normal}

Una alternativa interesante a este tipo de análisis de sensibilidad, es la de utilizar una distribución normal la cual es una de las distribuciones teóricas mejor estudiadas en los textos de estadística y más utilizada en la práctica, también llamada distribución gaussiana [101-103]. Su importancia se debe fundamentalmente a la frecuencia con la que distintas variables asociadas a fenómenos naturales y cotidianos siguen, aproximadamente, esta distribución. Caracteres morfológicos (como la talla o el peso), o psicológicos (como el cociente intelectual) son ejemplos de variables de las que frecuentemente se asume que siguen una distribución normal.

La distribución normal fue reconocida por primera vez por el francés Abraham de Moivre (1667-1754). Posteriormente, Carl Friedrich Gauss (1777-1855) elaboró desarrollos más profundos y formuló la ecuación de la curva; de ahí que también se le conozca, más comúnmente, como la "campana de Gauss". La distribución de una variable normal está completamente determinada por dos parámetros, su media y su desviación estándar, denotadas generalmente por $\mu$ y $\sigma$ [104]. Con esta notación, la densidad de la normal viene dada por la ecuación:

$$
f(x)=\frac{1}{\sigma \sqrt{2 \pi}} \exp \left\{\frac{-1}{2}\left(\frac{x-\mu}{\sigma}\right)^{2}\right\} ; \quad-\infty<x<\infty
$$

Puesto que la curva alcanza su mayor altura en torno a la media, mientras que sus ramas se extienden asintóticamente hacia los ejes, cuando una variable siga una distribución normal, será mucho más probable observar un dato cercano al valor medio que uno que se encuentre muy alejado de éste.

En este trabajo, los $O_{i}$ objetivos son los índices $P M V$ y $P P D$, que son una función de los diferentes parámetros mencionados en las secciones anteriores. Para analizar la influencia en el rendimiento térmico de los edificios, los parámetros $P_{j}$ son factor de vestimenta (clo) y factor de actividad (met) [15]. En especifico en este análisis de sensibilidad se opto por variar solo uno de los parámetros, manteniendo fijos el resto de ellos. Por ejemplo si variamos el factor de vestimenta, el factor de actividad permanece constante

\subsection{Cambio de categoría y temperatura de consigna}

Dependiendo de los rangos admisibles para PMV y PPD, la UNE-EN ISO 7730 define tres tipos de zonas de confort o categorías de requisitos térmicos como categoría A) $(P P D<6 \%$, es decir, $-0.2<P M V<0.2)$, categoría B $)(P P D$ 
$<10 \%$, es decir, $-0.5<P M V<0.5)$ y categoría $\mathbf{C}(P P D<15 \%$, es decir, $-0.7<P M V<0.7)$. El rango de temperaturas del aire recomendado para diferentes tipos de edificios, dependiendo de las categorías, se muestra en la Tabla 4.6 [15]. En base a los resultados del análisis de sensibilidad, podemos establecer la temperatura de referencia para las estaciones de verano e invierno.

\begin{tabular}{|c|c|c|c|c|}
\hline \multirow{2}{*}{$\begin{array}{l}\text { Tipo de } \\
\text { Edificios }\end{array}$} & \multirow{2}{*}{$\begin{array}{c}\text { Actividad } \\
\left(W / m^{2}\right)\end{array}$} & \multirow{2}{*}{ Categoría } & \multicolumn{2}{|c|}{ Temperatura $\left({ }^{\circ} C\right)$} \\
\hline & & & Verano & Invierno \\
\hline \multirow{3}{*}{$\begin{array}{l}\text { Aulas, oficinas } \\
\text { salas principales } \\
\text { salas de juntas }\end{array}$} & \multirow{3}{*}{70} & A & $24.5 \pm 1.0$ & $22 \pm 1.0$ \\
\hline & & B & $24.5 \pm 1.5$ & $22 \pm 2.0$ \\
\hline & & $\mathrm{C}$ & $24.5 \pm 2.5$ & $22 \pm 3.0$ \\
\hline
\end{tabular}

Tabla 4.6: Rango de temperatura de aire recomendado para oficinas y aulas (ISO 7730) [44].

La metodología propuesta en este trabajo consiste en obtener los rendimientos térmicos de los edificios con base en el análisis de sensibilidad para los parámetros subjetivos en el confort térmico, con el objetivo de identificar cuál de ellos tiene mayor influencia en las predicciones del modelo. 


\section{Capítulo 5}

\section{Resultados y discusión}

En esta sección presentamos los resultados de las simulaciones realizadas. Primero mostraremos la verificación del monitoreo utilizando los datos obtenidos por la plataforma Equs y por las simulaciones de cada edificio realizadas en TRNSYS. Luego, después de una segunda simulación, obtendremos la demanda de energía usando los resultados calculados para el cambio de categoría, dependiendo de la estimación de los valores de $P M V$ y $P P D$, asi como el análisis de sensibilidad de las variables del factor de vestimenta y factor de actividad. Finalmente, analizamos el impacto económico del ahorro en la demanda de energía.

Este trabajo une simulaciones de energía basadas en BIM y análisis de sensibilidad de dos edificios no residenciales en dos temporadas (verano e invierno) bajo estándares internacionales como ISO 7730 y ASHRAE 55.

\subsection{Monitorización en tiempo real: Equs}

El seguimiento en tiempo real en línea con Equs demuestra la confiabilidad de las mediciones que validan las simulaciones computacionales. En la Fig. 5.1 mostramos las temperaturas medidas desde el 1 de enero al 31 de octubre de 2015 de la zona de aire acondicionado del edificio de España, ubicada a $43^{\circ} 18^{\prime}$ de latitud norte y $8^{\circ} 17^{\prime}$ de longitud oeste. En el primer mes (enero, temporada de invierno) la temperatura más baja del período es $12.53^{\circ} \mathrm{C}$ registrado el sábado 17 . La temperatura más alta $30.48^{\circ} \mathrm{C}$ se alcanzó el lunes 15 de junio (verano). La temperatura media fue de $22.43^{\circ} \mathrm{C}$. En el caso de la temperatura exterior, observamos que las temperaturas frías y calientes están entre $2^{\circ} \mathrm{C}$ y $41^{\circ} \mathrm{C}$, aproximadamente. La temperatura media fuera del edificio fue de $15.49^{\circ} \mathrm{C}$. 


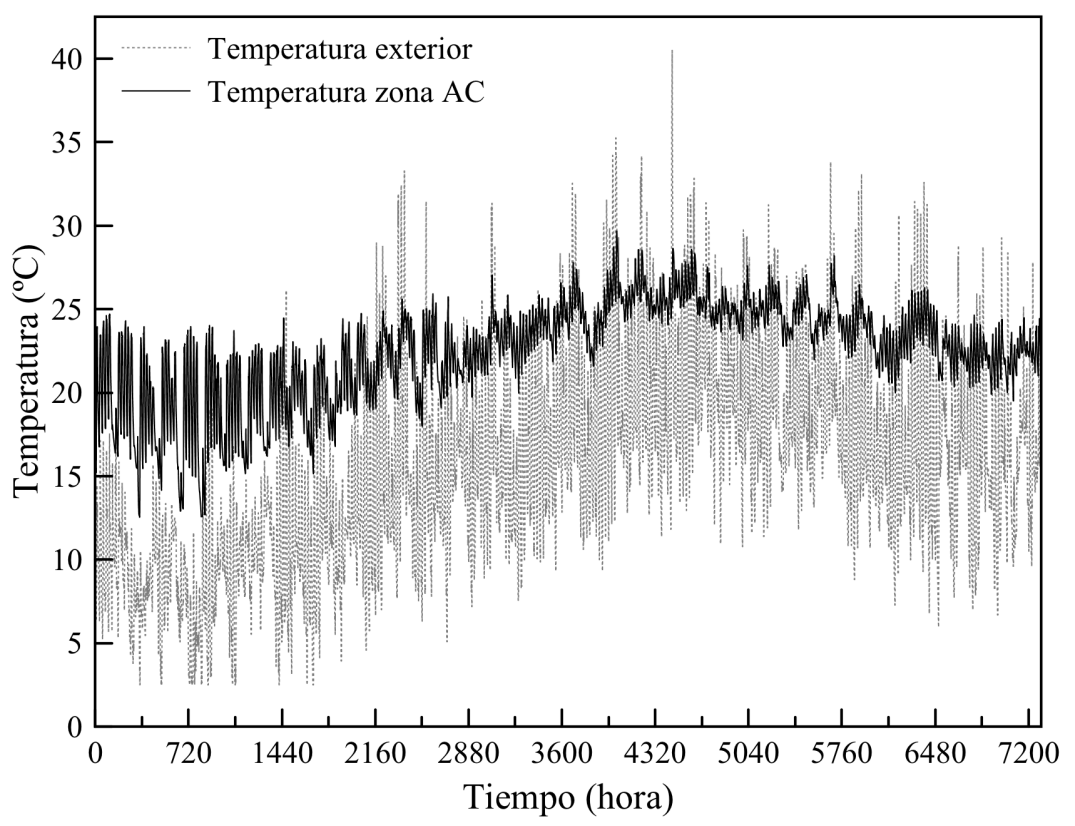

Figura 5.1: Temperaturas internas y externas medidas por la plataforma web Equs. En el caso de la temperatura exterior, observamos que las temperaturas frías y calientes están entre $2^{\circ} \mathrm{C}$ y $41^{\circ} \mathrm{C}$, aproximadamente. La temperatura promedio fue de $15.49^{\circ} \mathrm{C}$. 


\subsection{Validación de simulación en TRNSYS}

La comparación entre los datos reales y las simulaciones es necesaria para tener modelos confiables de los edificios. Como ejemplo, el paralelismo entre datos experimentales y el modelado de la temperatura del aire interno para el mes de junio, desde la hora 3624 a 4344, se muestra en la Fig. 5.2(a). Para el período de junio, la diferencia máxima entre las temperaturas simuladas y medidas es $1.47^{\circ} \mathrm{C}$, siendo la desviación media $0.49^{\circ} \mathrm{C}$. Estos valores no tienen influencia con respecto a la comodidad interior, lo que demuestra que los resultados de nuestro modelo son esencialmente correctos.

En la Fig. 5.2(b) mostramos la temperatura de un solo día de junio (24h desde la hora 3914 a 3938 de la simulación). Podemos observar con más detalle la diferencia entre la temperatura calculada y la medida para el período mencionado, donde la variación mínima es de $0.04^{\circ} \mathrm{C}$ (obtenida en horas de trabajo) y la diferencia media (promedio) es de $0.35^{\circ} \mathrm{C}$. La variación máxima es $0.72^{\circ} \mathrm{C}$. Estos resultados se obtuvieron dentro de la situación descrita en secciones anteriores. Particularmente, durante los períodos de ocupación, la tasa metabólica (met) de los ocupantes se seleccionó como "Trabajos ligeros sentados", que corresponde a $70 \mathrm{~W} / \mathrm{m}^{2}$ o 1.2 met. El factor de vestimenta (clo) se estableció en 0.7 para el período de verano y 1.2 para el invierno. Tengamos en cuenta que una ropa con factor de 1.2 clo corresponde a: ropa interior, camisa, pantalones, chaqueta térmica, calcetines y zapatos. La ropa de verano de 0.7 clo corresponde a: ropa interior, la camisa, los pantalones, los calcetines y los zapatos (ISO 7730). La Tabla 4.5 muestra la entrada que se usó para la simulación para el escenario del caso inicial. En la Fig. 5.3 podemos observar la demanda de energía en la configuración inicial, donde el punto de referencia se estableció en $23.8^{\circ} \mathrm{C}$.

El objetivo principal de la aplicación de un análisis de sensibilidad en este trabajo es visualizar, de una manera más simple y detallada, el impacto de las decisiones subjetivas tomadas por el diseñador sobre los valores de factor de vestimenta o aislamiento de ropa y el factor de actividad o factor metabólico para el confort térmico, que se definen en el programa de simulación TRNSYS. Basado en ISO 7730, el factor de vestimenta(clo) se puede estimar directamente a partir de la combinación de la ropa o agregando los valores de aislamiento parcial para cada prenda. Por ejemplo, el valor típico de aislamiento de ropa para el verano (refrigeración) y el invierno (calefacción) es de 0.5 y 1.0 clo, respectivamente. Por tanto, los valor asociados a cada prenda pueden ser determinantes en la asignación del valor del factor de vestimenta $(\mathrm{clo})$ por parte del diseñador y su determinación está sujeto, por tanto a más fuentes de error.

Por ejemplo, el aislamiento de ropa ligera de verano de 0.5 clo incluye prendas 


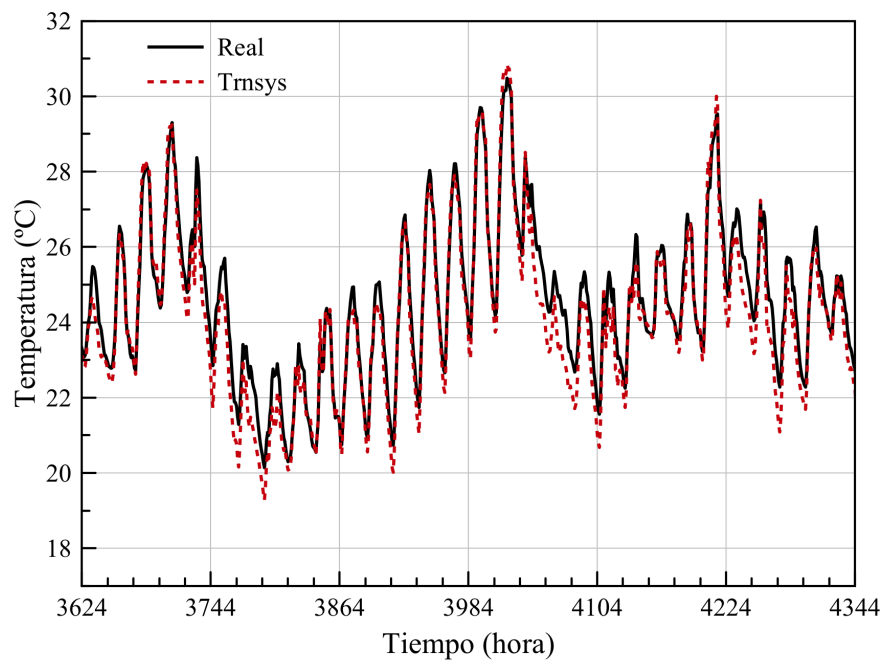

(a)

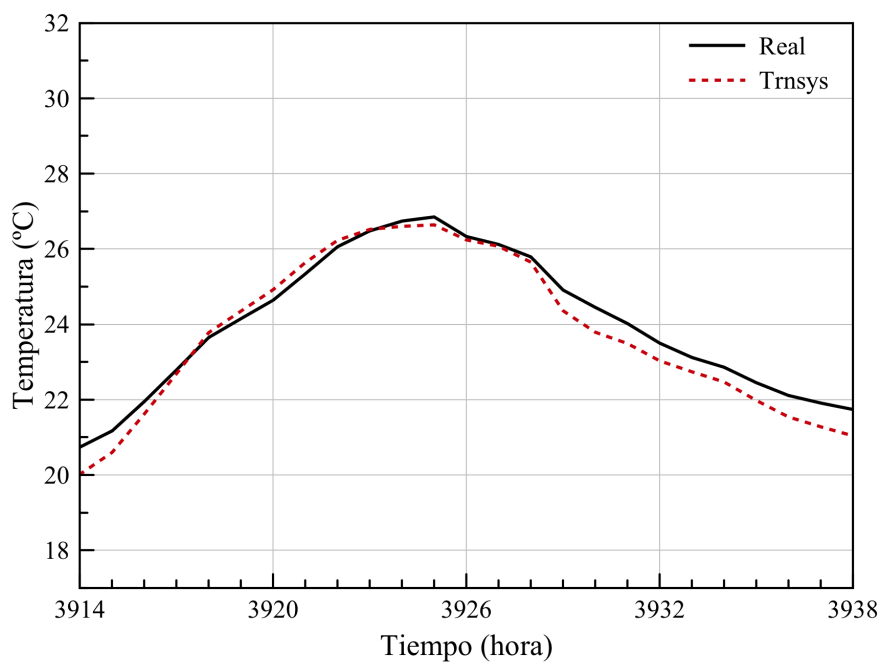

(b)

Figura 5.2: Comparación entre los datos reales obtenidos con Equs y los resultados de simulaciones teóricas del primer caso de estudio: (a) para el mes de junio y (b) un solo día de junio. 


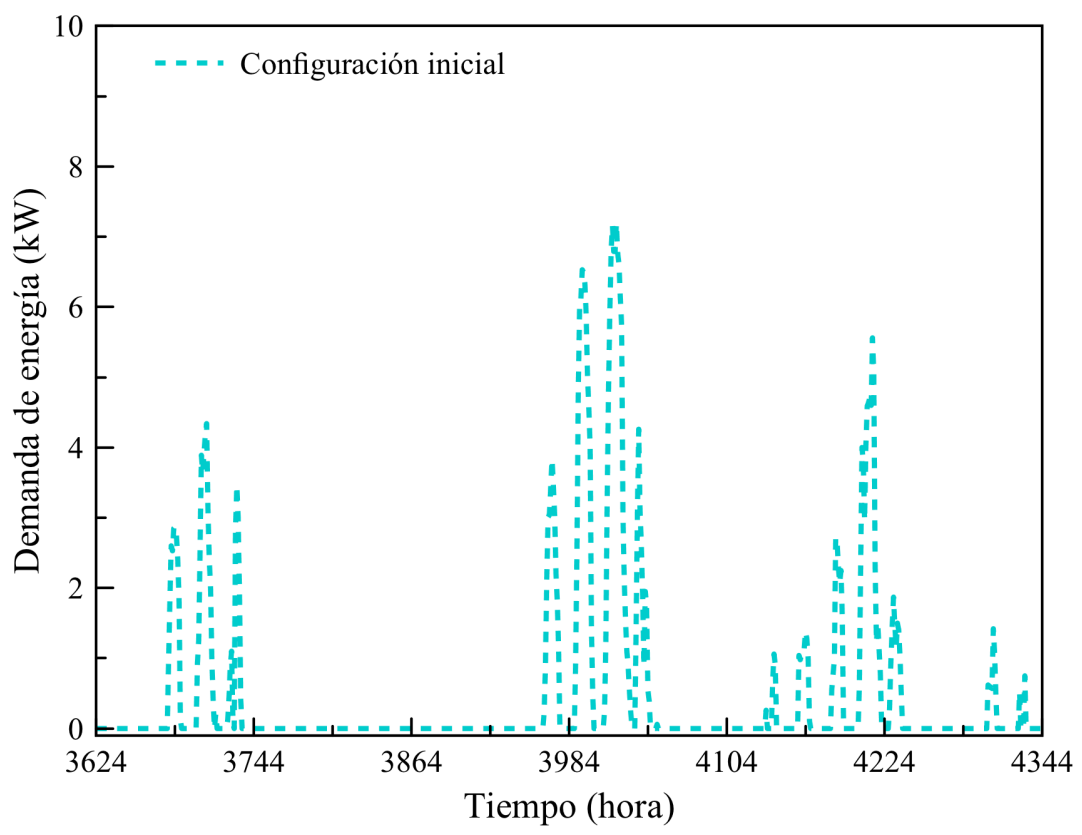

Figura 5.3: Demanda de energía del edificio (caso España) en la configuración inicial de los parámetros de confort térmico. 
como: calzoncillos, camisa de manga corta, pantalones ligeros, calcetines delgados o zapatos. En nuestro caso, elegimos para ambos casos de estudio un valor de 0.7 clo que incluye: ropa interior, camisa, pantalones, calcetines y zapatos, que también se consideran ropa ligera adecuada para la misma temporada en este tipo de edificios. Análogamente, la estimación del valor de la actividad o tasa metabólica (met), que representa una producción de calor en función del nivel de actividad, también depende de la decisión subjetiva realizada por el diseñador. Según la norma ISO 7730 para una actividad correspondiente a este tipo de edificios, como: oficinas, escuelas y laboratorios, el parámetro de actividad puede variar de 1.0 a 1.6 met. Para este trabajo asumimos un valor de 1.2 met para las simulaciones de energía en TRNSYS.

\subsection{Análisis de sensibilidad mediante el método de Monte Carlo}

Por tanto, es de fundamental importancia estimar el impacto de las elecciones subjetivas mencionadas de vestimenta y factor metabólico, sobre los valores de los índices $P M V$ y $P P D$, que indican el nivel de confort térmico y criterios de confort térmico local, indicando las condiciones ambientales que se consideran aceptables para el confort térmico general, así como malestar local.

Teniendo en cuenta lo anterior, utilizamos el análisis de sensibilidad basado en el método de Monte Carlo, generando dos diferentes distribuciones aleatoriamente: distribución uniforme y distribución normal.

\subsubsection{Distribución uniforme}

Se generó una distribución uniforme para una variación del factor de vestimenta de 0 a $2 c l o$, y el factor de actividad desde 0.8 a 4 met, ya que en este rango el índice $P M V$ se encuentra entre -2 y 2 , teniendo en cuenta que este es el rango sugerido por la normativa ISO 7730. Los resultados del SA en los índices de confort (PMV y $P P D$ ) para el factor de vestimenta y la tasa metabólica se muestran en las Figs. 5.4 y 5.5, respectivamente. La categoría térmica cambia según los valores de $P M V$ y $P P D$ que, a su vez, dependen de las decisiones subjetivas por parte del diseñador para el factor de vestimenta y el factor de actividad. La Fig. 5.4 muestra que para la categoría $\mathrm{A}$, los valores del factor de vestimenta varían de 0.46 a 0.66 clo, para $\mathrm{B}$ de 0.34 a 0.46 clo y de 0.66 a 0.87 clo. Finalmente, la categoría $\mathrm{C}$ está entre 0.27 a 0.34 clo y 0.87 a 1.04 clo. En este caso, el factor de actividad permanece constante con un valor de 1.2 met.

Para el caso de met y PPD, en la Fig. 5.5 mostramos que la categoría A se encuen- 
tra entre los valores de 0.83 a 1.04 met. Para el caso de la categoría $\mathrm{B}$, permanece de 0.8 a 0.83 met y de 1.04 a 1.16 met. Finalmente, la categoría $\mathrm{C}$ corresponde al rango de 1.16 a 1.31 met. En este caso en particular el factor de vestimenta permanece constante a 0.7 clo.

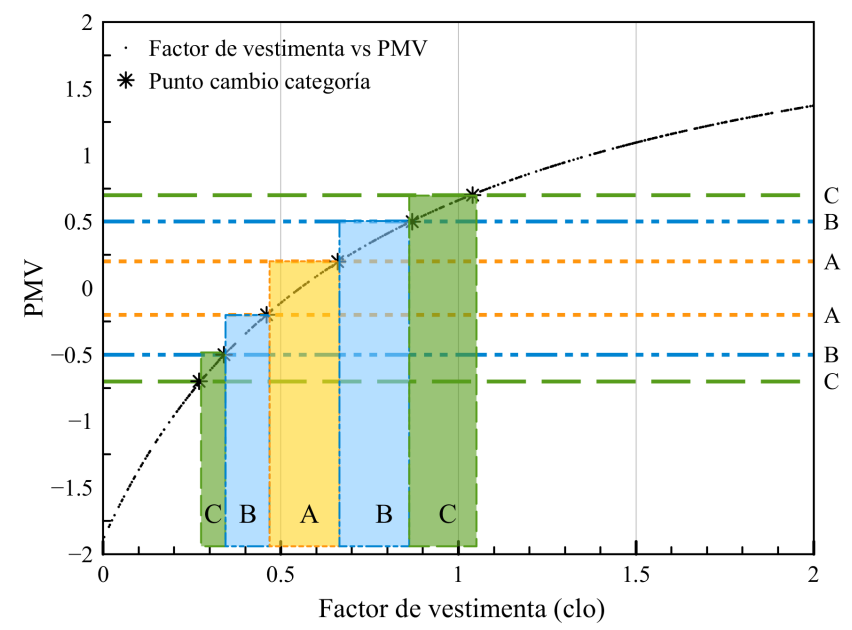

Figura 5.4: Cambio del nivel de confort térmico en base al análisis de sensibilidad del factor de vestimenta en $P M V$ (factor de actividad permanece constante).

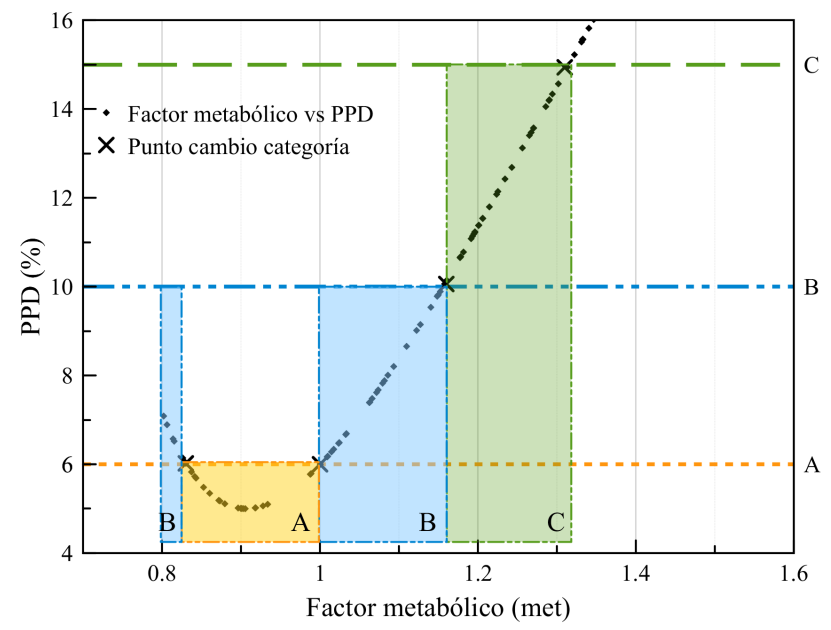

Figura 5.5: Cambio del nivel de confort térmico en base al análisis de sensibilidad del factor metabólico en PPD (factor de vestimenta permanece constante).

Debemos notar que una variación en los valores del factor de vestimenta en ve- 
rano (Fig. 5.4), de 0.3 a $0.8 c l o$, causa un cambio en la categoría de A a C.

\subsubsection{Distribución normal}

Para el caso de una distribución normal, hemos generado aleatoriamente valores para el factor de vestimenta con $\mu=1.0 \mathrm{clo}$, fijando el factor metabólico a 100 $\operatorname{met}(*) \mathrm{y}$, variando la desviación típica $\sigma_{c l o}=0.05,0.075,0.1,0.125$ y 0.15 . Para estas variaciones en los valores de entrada obtenemos (a través de nuestro modelo) las variaciones en las variables de salida $P M V$ y $P P D$. Mediante un ajuste a una

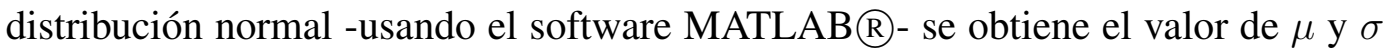
de sus distribuciones.

En las Figs. 5.6 y 5.7 se observan los sucesos del factor de vestimenta y factor de actividad generados aleatoriamente siguiendo las distribuciones uniforme y normal.
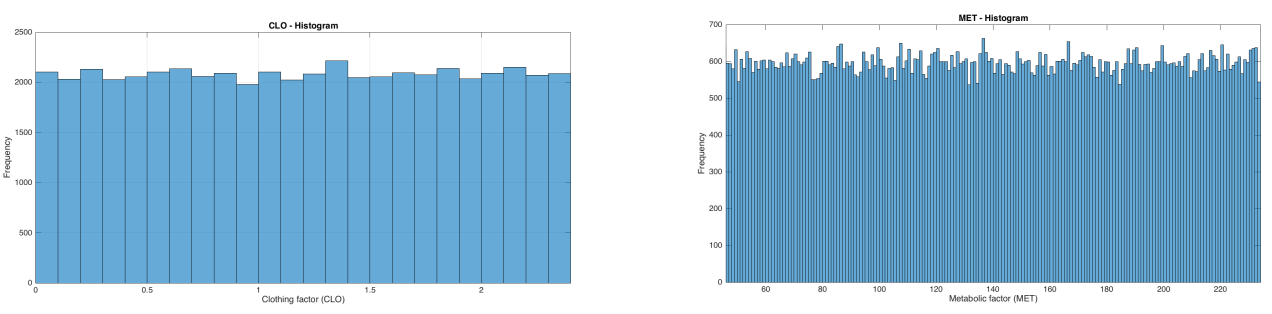

Figura 5.6: Generación de sucesos distribuidos uniformemente para los parámetros de factor de vestimenta (clo) y factor metabólico (met).
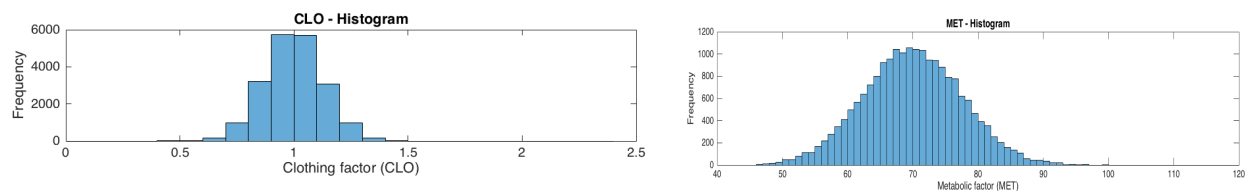

Figura 5.7: Generación de sucesos con base a distribución normal para los parámetros de factor de vestimenta (clo) y factor metabólico (met).

La Fig. 5.8(a) muestra la relación entre $\sigma_{c l o}$ y $\sigma_{P M V}$ observamos que la dependencia es lineal y mediante una regresión obtenemos una pendiente de 0.73 y un coeficiente de determinacion $R^{2}=0.999$; para la Fig. 5.8(b), tenemos una recta con pendiente de 33.042 y $R^{2}=1.0$ para el caso de $\sigma_{\text {clo }}$ y $\sigma_{P P D}$.

* El factor de actividad se suele expresar en unidades met. 1 met corresponde a una cantidad metabólica de $58.15 \mathrm{~W} / \mathrm{m}^{2}$. En este epígrafe tomamos las unidades de $W / m^{2}$ y, por tanto, $\mu=100$ y $\sigma_{m e t}=2.5,5,7.5,10$ y 12.5 corresponden a $\mu=100 \mathrm{~W} / \mathrm{m}^{2}$ y $\sigma_{\text {met }}=2.5 \mathrm{~W} / \mathrm{m}^{2}, 5 \mathrm{~W} / \mathrm{m}^{2}, 7.5 \mathrm{~W} / \mathrm{m}^{2}, 10 \mathrm{~W} / \mathrm{m}^{2}$ y $12.5 \mathrm{~W} / \mathrm{m}^{2}$. 
De manera similar, para el caso del factor de actividad (met) se ha establecido una $\mu=100$, fijando factor de vestimenta a 1.0clo y variando la desviación típica $\sigma_{m e t}=2.5,5,7.5,10$ y 12.5 . Las rectas de la Fig. 5.9, muestran la relación entre el $\sigma_{m e t}$ y (a) $\sigma_{P M V}$ : una recta con pendiente de 1.43 y $R^{2}=0.999$ y, para (b) $\sigma_{P P D}$ : una recta con pendiente de 65.59 y $R^{2}=0.999$.

En las siguientes figuras 5.8 y 5.9, se muestra la relación entre las sigmas del PMV y PPDcon las sigmas de clo y met.
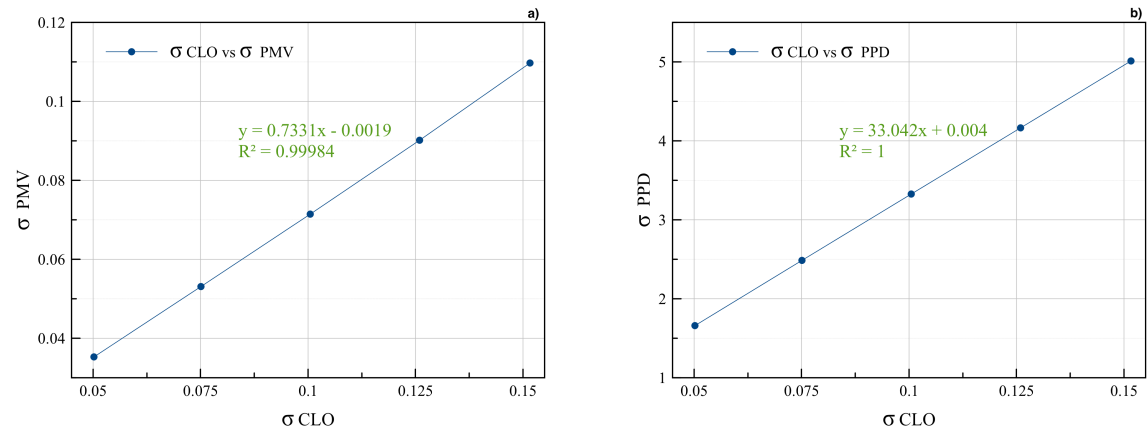

Figura 5.8: Relación en base al análisis de sensibilidad del factor de vestimenta $\left(\sigma_{c l o}\right):(\mathrm{a}) \sigma_{P M V}$ y $(\mathrm{b}) \sigma_{P P D}$.
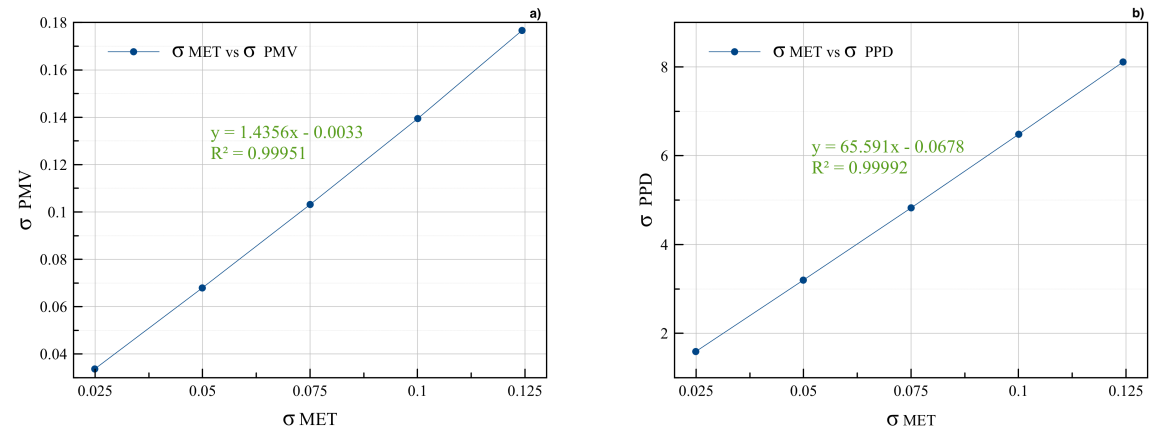

Figura 5.9: Relación en base al análisis de sensibilidad del factor metabólico $\left(\sigma_{m e t}\right)$ : (a) $\sigma_{P M V}$ y (b) $\sigma_{\mathbf{P P D}}$.

Es decir, hemos obtenido la relación de las variaciones del factor de vestimenta $(c l o)$ y factor de actividad (met) con las anchuras $(\sigma)$ del $P M V$ y $P P D$, con el objetivo de observar cual de los dos parámetros que ingresa el diseñador de manera subjetiva, produce un mayor impacto en la salida del modelo de la estimación de los indices del $P M V$ y $P P D$. 
En la figura 5.10, se muestra simultaneamente las anchuras de $P M V \sigma_{P M V}$ en función de la anchura del factor de vestimenta (dejando el factor de vestimenta constante)y el factor metabólico (manteniendo esta vez el valor de clo constante). Por ejemplo, para una anchura del $5 \%$ respecto al valor central del factor de vestimenta $\mu=1$ clo $\left(\sigma_{c l o}=0.05\right)$, se obtiene un valor de $\sigma_{P M V}$ de 0.035 y para una anchura del $5 \%$ respecto al valor central del factor metabólico $\mu=100$ met $\left(\sigma_{m e t}=5\right)$, se obtiene un valor de $\sigma_{P M V}$ de 0.068 . Además observamos que el valor de la pendiente del factor de actividad (0.0143) es mayor a la pendiente del factor de vestimenta (0.0073). Por tanto, el impacto del factor metabólico (met) es mayor que el del factor de vestimenta (clo) para el caso del PMV.

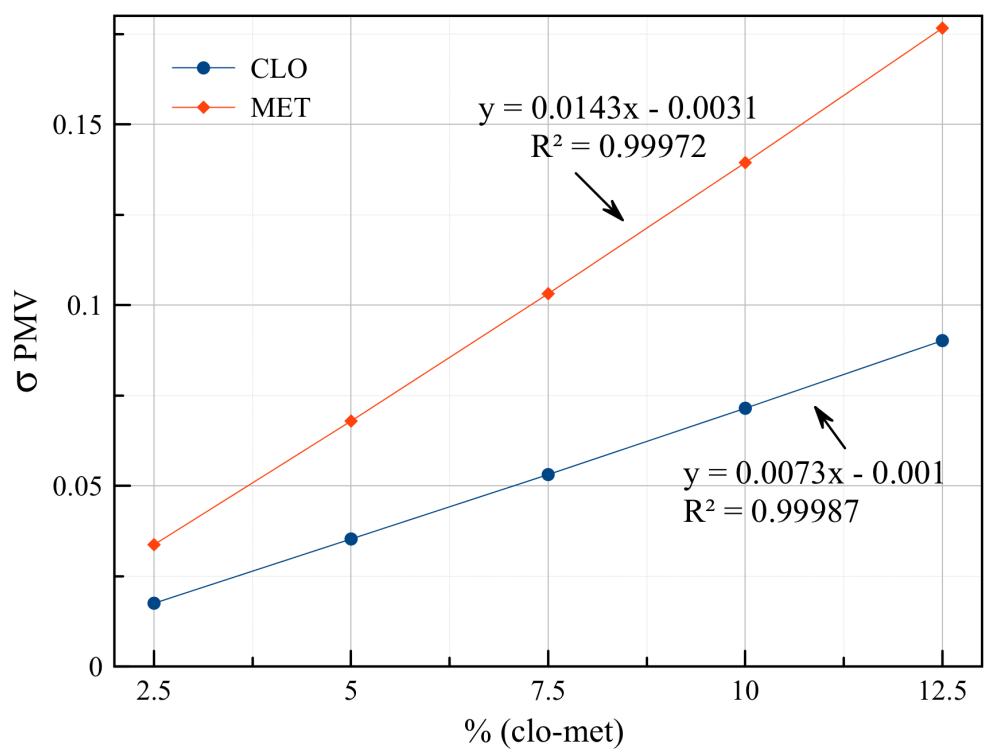

Figura 5.10: Relación entre las variaciones del factor de vestimenta (clo) y factor de actividad (met) y las anchuras de $P M V$.

De manera idéntica, para cada porcentaje de variación en los valores de factor de vestimenta (clo) y factor de actividad (met) tenemos un valor de $\sigma_{P P D}$, que se muestra en la figura 5.11, por ejemplo: para una anchura del $12.5 \%$ el valor de $\sigma_{P P D}$ es de 4.166 para una variación en el factor de vestimenta $(c l o)$ y de 8.111 si es el parámetro del factor de actividad (met) el que varía. Además, los valores de las pendientes 0.6532 y 0.3339 para el factor de vestimenta y metabólico, respectivamente, también predicen que el impacto del factor metabólico (met) es mayor que el factor de vestimenta (clo) en el $P P D$. 


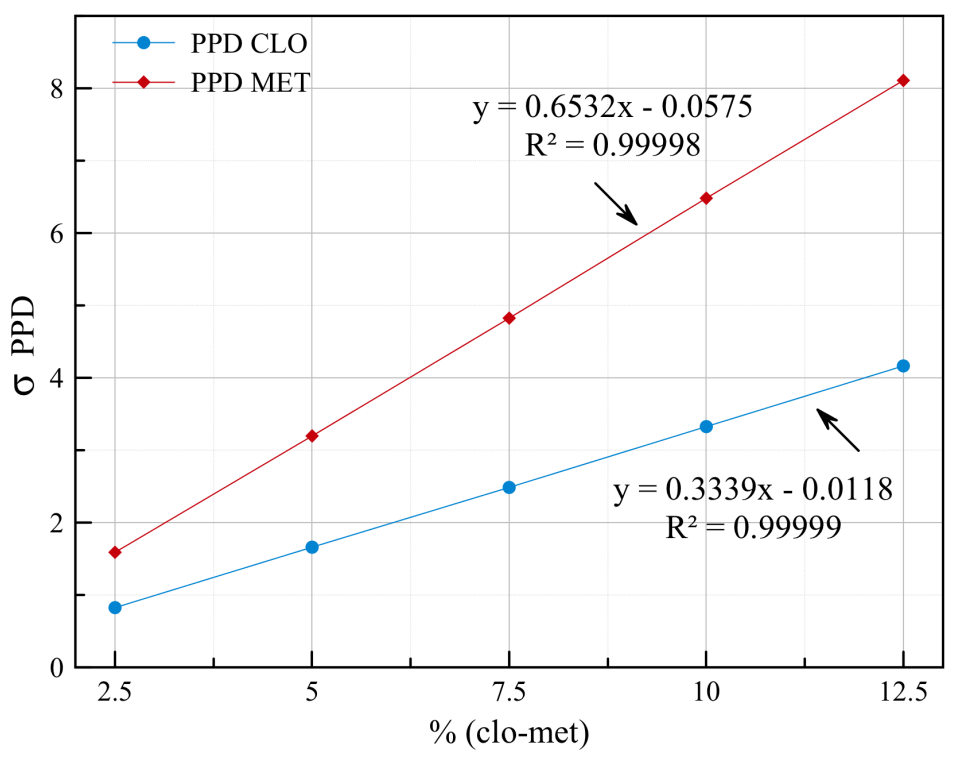

Figura 5.11: Relación entre las variaciones del facto de vestimenta ( clo) y el factor metabólico (met) y las anchuras de PPD.

\subsection{Demandas energéticas e impacto económico}

De acuerdo con los resultados de SA y los criterios de la tabla 4.6, se estableció para la simulación en TRNSYS los valores del factor vestimenta (clo) y el factor de actividad (met) y las temperaturas interiores de consigna.

La primer configuración se estableció para un valor del factor vestimenta de 0.7 clo con una temperatura de $23.5^{\circ} \mathrm{C}$, que pertenece a la categoría A. Para la categoría B, usamos una temperatura de $24^{\circ} \mathrm{C}$ y $0.5 \mathrm{clo}$. Finalmente, se consideró una temperatura de punto de ajuste de $25^{\circ} \mathrm{C}$ para la categoría $\mathrm{C}$ y $0.3 \mathrm{clo}$. Como ejemplo, para el mes de junio, de la Fig. 5.12 para valores del factor vestimenta de 0.3, 0.5 y 0.7 clo obtenemos una demanda total de energía de $347.19 \mathrm{~kW}, 565.05 \mathrm{~kW}$ y $885.2 \mathrm{~kW}$, respectivamente. En el caso del factor de actividad, se procede de igual modo que en el caso de la vestimenta, introduciendo en TRNSYS los nuevos valores de temperatura alcanzada y establecida en función del rango de temperatura del aire acondicionado.

En la Fig. 5.12, se muestra la variación de la demanda de energía con respecto al cambio en la categoría ambiental. 


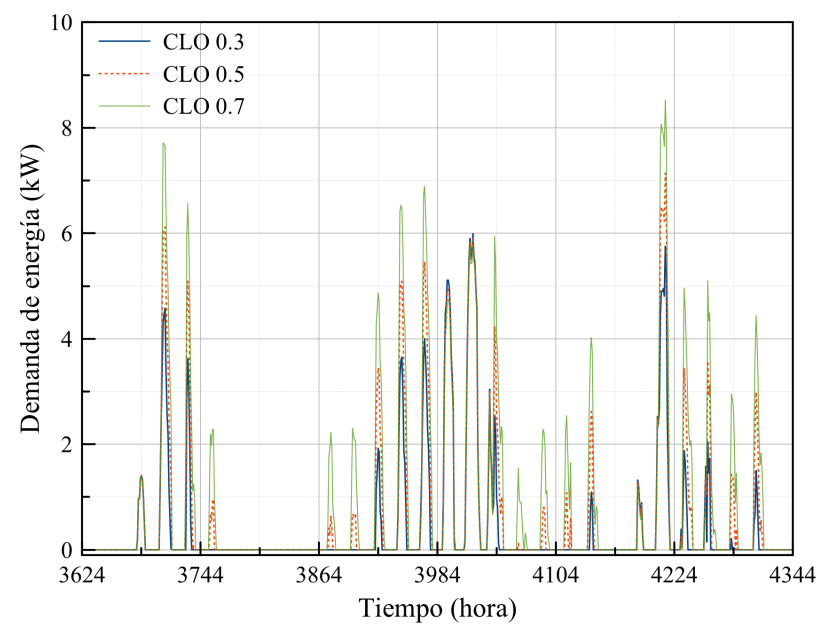

Figura 5.12: Demanda de energía para varios valores de factor de vestimenta.

\begin{tabular}{|c|c||c|c|c||c|c|c|}
\hline Caso & \multicolumn{1}{|c||}{$\begin{array}{c}\text { Temp. } \\
\text { Consig. }\end{array}$} & \multicolumn{6}{|c|}{$\begin{array}{c}\text { Demanda de energía acumulada (kW) } \\
\text { para clo \& met (junio a septiembre) }\end{array}$} \\
\cline { 3 - 8 } & & 0.3 clo & 0.5 clo & 0.7 clo & 0.8 met & 1.0 met & 1.2 met \\
\hline \hline España & $23^{\circ} \mathrm{C}$ & 10035.15 & 10103.31 & 10145.80 & 10474.85 & 10514.24 & 10656.33 \\
\hline España & $24^{\circ} \mathrm{C}$ & 9744.55 & 9747.20 & 9796.10 & 10166.64 & 10190.55 & 10216.24 \\
\hline España & $25^{\circ} \mathrm{C}$ & 9566.70 & 9581.64 & 9595.09 & 9998.72 & 10010.56 & 10023.37 \\
\hline \hline México & $23^{\circ} \mathrm{C}$ & 273158.46 & 275990.91 & 278876.27 & 343595.12 & 349307.2 & 355060.52 \\
\hline México & $24^{\circ} \mathrm{C}$ & 247775.81 & 250057.84 & 252401.52 & 288935.87 & 294159.43 & 299428.98 \\
\hline México & $25^{\circ} \mathrm{C}$ & 227789.41 & 229537.78 & 231344.40 & 242446.35 & 244186.78 & 248938.99 \\
\hline
\end{tabular}

Tabla 5.1: Demanda acumulada de energía entre junio y septiembre, con valores variables de clo y met, para diferentes temperaturas.

En la Tabla 5.1 se pueden observar que los cambios en la demanda de energía debido a variaciones en los criterios del diseñador en un edificio como el situado en México, pueden variar hasta un $2.09 \%$ en el caso de clo y un $3.63 \%$ para met [105]. Análogamente, para el edificio en España, la variación del factor metabólico produce un mayor impacto en la demanda energética que la variación en el factor de vestimenta. De hecho, la variación en un $133 \%$ del factor de vestimenta (de 0.3 clo a 0.7 clo) produce una variación en la demanda de $1.10 \%$ mientras que una variación del $50 \%$ en el factor metabólico (de 0.8 met a 1.2 met) produce un incremento en la demanda de energía del $1.73 \%$. Para ambos casos de estudio, la variación de los factores de actividad y vestimenta presentan una variación en la demanda de energía que a su vez se traduce en consumo energético y económico. Este último dependerá de los costos locales de la energía eléctrica. 


\section{Capítulo 6}

\section{Conclusiones}

Se ha demostrado que la decisión del diseñador en relación a la asignación del valor del factor de vestimenta (entre 0.3 clo y 0.7 clo) o del factor metabólico (entre 0.8 met y 1.2 met) tiene un importante efecto energético. De hecho, se ha analizado el impacto en la demanda energética para diferentes temperaturas de consigna $\left(T=23^{\circ} \mathrm{C}, 24^{\circ} \mathrm{C}\right.$ y $25^{\circ} \mathrm{C}$ ). En cualquier caso, lo mas relevante de este trabajo de investigación es proponer una metodología facilmente extrapolable a edificios con clima, uso y estructura diferentes.

Los resultados indican que el efecto en la demanda energética al variar el factor de vestimenta en el edificio de México llega a alcanzar un $2.09 \%$ y la variación en el factor metabólico alcanza el $3.63 \%$. En el caso del edificio situado en España, y debido a la diferente tipología y ubicación del edificio, la variación en el factor de vestimenta es del $1.1 \%$ y el efecto del factor metabólico del $1.73 \%$.

En definitiva, nuestro análisis ha permitido corroborar las hipótesis planteadas que destacaban la importancia de las variables de decisión subjetiva por parte del diseñador (de acuerdo con la norma ISO 7730) en el desempeño energético del edificio.

Ya que la ISO 7730 es una norma internacional, debido a la gran variedad de climas existentes, es muy importante tener en cuenta esta peculiaridad del sistema en el momento de su aplicación y en la elección del diseñador, que debe estar familiarizado con el procedimiento. La norma internacional ISO 7730 es referencia en todas las regulaciones del mundo y, sin embargo, requiere de un diseñador experimentado para una aplicación adecuada. Por tanto, hemos mostrado en el presente trabajo el gran impacto de las elecciones subjetivas (actividad o vestimenta) del diseñador en el cálculo de la demanda de energía de los edificios, en base al análisis de sensibilidad basado en la norma ergonómica ambiental ISO 7730, el cual concluye que 
la elección del met presenta una mayor sensibilidad, causando un aumento significativo en el consumo de energía, así como los costos adicionales acumulados para garantizar la comodidad de sus ocupantes.

Además, a través del análisis de sensibilidad Monte Carlo, se ha concluido la mayor influencia del factor metabólico respecto al factor de vestimenta en las variables relacionadas con el confort: $P M V$ y $P P D$. 


\section{Bibliografía}

[1] C. Optimality. Discussing methodology and challenges within the recast energy performance of buildings directive. Buildings Performance Institute Europe (BPIE): Brussels, Belgium, 2010.

[2] EUROSTAT, Energy - yearly statistics 2015., 2015.

[3] R.K. Pachauri, L. Meyer, Van K.L. Brinkman, S., N. Leprince-Ringuet, and Van B.F. Cambio climático 2014 Equipo principal de redacción. Unidad de apoyo técnico para el Informe de síntesis.

[4] T.L. Hemsath and K.A Bandhosseini. Sensitivity analysis evaluating basic building geometry's effect on energy use. Renewable Energy, 76:526-538, 2015.

[5] D. Griego, M. Krarti, and A. Hernandez-Guerrero. Energy efficiency optimization of new and existing office buildings in Guanajuato, Mexico. Sustainable Cities and Society, 17:132-140, 2015.

[6] H.W. Lin and T. Hong. On variations of space-heating energy use in office buildings. Applied Energy, 111:515-528, 2013.

[7] E. Pikas, M. Thalfeldt, and J. Kurnitski. Cost optimal and nearly zero energy building solutions for office buildings. Energy and Buildings, 74:30-42, 2014.

[8] J. Terés-Zubiaga, A. Campos-Celador, I. González-Pino, and C. EscuderoRevilla. Energy and economic assessment of the envelope retrofitting in residential buildings in northern spain. Energy and buildings, 86:194-202, 2015.

[9] J. Lee, J. Kim, D. Song, J. Kim, and C. Jang. Impact of external insulation and internal thermal density upon energy consumption of buildings in a temperate climate with four distinct seasons. Renewable and Sustainable Energy Reviews, 75:1081-1088, 2017. 
[10] D. Kolokotsa, D. Tsiavos, G.S. Stavrakakis, K. Kalaitzakis, and E. Antonidakis. Advanced fuzzy logic controllers design and evaluation for buildings' occupants thermal-visual comfort and indoor air quality satisfaction. Energy and buildings, 33(6):531-543, 2001.

[11] C.A. Balaras, K. Droutsa, E. Dascalaki, and S. Kontoyiannidis. Heating energy consumption and resulting environmental impact of european apartment buildings. Energy and buildings, 37(5):429-442, 2005.

[12] L. Pérez-Lombard, J. Ortiz, and C. Pout. A review on buildings energy consumption information. Energy and buildings, 40(3):394-398, 2008.

[13] N. Jestin-Fleury. International Energy Agency. World Energy Outlook. Politique étrangère, 59(2):564-565, 1994.

[14] J.E. Anderson, G. Wulfhorst, and W. Lang. Energy analysis of the built environment - A review and outlook. Renewable and Sustainable Energy Reviews, 44:149-158, 2015.

[15] E.A. Abdelaziz, R. Saidur, and S. Mekhilef. A review on energy saving strategies in industrial sector. Renewable and sustainable energy reviews, 15(1):150-168, 2011.

[16] P. Nejat, F. Jomehzadeh, M.M. Taheri, M. Gohari, and M.Z.A. Majid. A global review of energy consumption, co2 emissions and policy in the residential sector (with an overview of the top ten co2 emitting countries). Renewable and sustainable energy reviews, 43:843-862, 2015.

[17] R. Galvin. Thermal upgrades of existing homes in Germany: The building code, subsidies, and economic efficiency. Energy and Buildings, 42(6):834$844,2010$.

[18] J.C. Creyts. Reducing US greenhouse gas emissions: how much at what cost?: US Greenhouse Gas Abatement Mapping Initiative. McKinsey \& Co., 2007.

[19] G. Cooper. Air-conditioning America: engineers and the controlled environment, 1900-1960. Number 23. JHU Press, 2002.

[20] H. Chappells and E. Shove. Debating the future of comfort: environmental sustainability, energy consumption and the indoor environment. Building Research \& Information, 33(1):32-40, 2005.

[21] R. Goldsmith. Use of clothing records to demonstrate acclimatization to cold in man. Journal of applied Physiology, 15(5):776-780, 1960. 
[22] J.F. Nicol, I.A. Raja, A. Allaudin, and G.N. Jamy. Climatic variations in comfortable temperatures: the pakistan projects. Energy and buildings, 30(3):261-279, 1999.

[23] H. B. Awbi. Ventilation of buildings. Routledge, 2002.

[24] A. Hernández. NTP 343: Nuevos criterios para futuros estándares de ventilación de interiores.

[25] S. Atthajariyakul and T. Leephakpreeda. Real-time determination of optimal indoor-air condition for thermal comfort, air quality and efficient energy usage. Energy and Buildings, 36(7):720-733, 2004.

[26] M. Castilla, J.D. Álvarez, M. Berenguel, M. Pérez, F. Rodríguez, and J.L. Guzmán. Técnicas de control del confort en edificios. Revista Iberoamericana de Automática e Informática Industrial RIAI, 7(3):5-24, 2010.

[27] M.C. Hernández-Luna, R. Robledo-Fava, P. de Fernández de Córdoba, A. Paredes, H. Michinel, and S. Zaragoza. Use of statistical correlation for energy management in office premises adopting techniques of the industry 4.0. Aceptado y pendiente de publicar.

[28] A. Hernández. NTP 742: Ventilación general de edificios. Instituto Nacional de Seguridad e Higiene en el Trabajo Ministerio de Trabajo y Asuntos Sociales, 1994.

[29] S. Atthajariyakul and T. Leephakpreeda. Neural computing thermal comfort index for hvac systems. Energy Conversion and Management, 46(1516):2553-2565, 2005.

[30] Turismo y Comercio de España Ministerio de Industria. Reglamento de instalaciones térmicas en los edificios. technical report. Technical report, IDAE, 2007.

[31] J. Guasch, R. Forster, F. Ramos, A. Hernández, and N.A. Smith. Enciclopedia de salud y seguridad en el trabajo: Iluminación. Organización Internacional del Trabajo. Ministerio de Trabajo y Asuntos Sociales, Tech. Rep, 2001.

[32] A.K. Yener. A method of obtaining visual comfort using fixed shading devices in rooms. Building and Environment, 34(3):285-291, 1998.

[33] Society of Light, Lighting, and Chartered Institution of Building Services Engineers. Code for Lighting. Butterworth-Heinemann, 2002.

[34] N. Española. UNE-EN 12464-1 febrero 2003. Iluminación de los lugares de trabajo. 
[35] N. Española. UNE-EN 12464-2 2008. Iluminación de los lugares de trabajo.

[36] G.K. Oral, A.K. Yener, and N.T. Bayazit. Building envelope design with the objective to ensure thermal, visual and acoustic comfort conditions. Building and Environment, 39(3):281-287, 2004.

[37] J.T. Kim and G. Kim. Overview and new developments in optical daylighting systems for building a healthy indoor environment. Building and Environment, 45(2):256-269, 2010.

[38] CARM.es - Confort acústico. https : / /www. carm. es/web/, 21 de junio de 2018.

[39] A. Hernández. NTP 503: Confort acústico: el ruido en oficinas. INSHT. Barcelona, 1998.

[40] A.C. Balagué and J.L. González. Una norma ambigua: La NBE CA-82 aprobada. CAU: construcción, arquitectura, urbanismo, (81):43, 1982.

[41] ASHRAE 1989. Standard 62-1989. Ventilation for acceptable indoor air quality, 41:51, 1989.

[42] J.W. MacArthur, E. Arens, R. Gonzalez, L. Berglund, S. Spain, T. Madsen, B. Oleson, and K. Reid. Hvac is for people. ASHRAE transactions, 92:5-64, 1986.

[43] P.O. Fanger. Thermal environment - human requirements. Environmentalist, 6(4):275-278, 1986.

[44] CEN (European Committee for Standardization). ISO 7730: Ergonomics of the thermal environment analytical determination and interpretation of thermal comfort using calculation of the PMV and PPD indices and local thermal comfort criteria, 2005.

[45] ASHRAE Standard. ASHRAE standard 55. Thermal environmental conditions for human occupancy. Atlanta: American Society of Heating, Refrigerating, and Air-Conditioning Engineers, 2010.

[46] ASHRAE. Handbook of fundamentals. American Society of Heating Refrigerating and Air Conditioning Engineers, Atlanta, GA, 2005.

[47] A. Geva, H. Saaroni, and J. Morris. Measurements and simulations of thermal comfort: a synagogue in Tel Aviv, Israel. Journal of Building Performance Simulation, 7(3):233-250, 2014. 
[48] A.T. Nguyen and S. Reiter. Passive designs and strategies for low-cost housing using simulation-based optimization and different thermal comfort criteria. Journal of Building Performance Simulation, 7(1):68-81, 2014.

[49] W. Wardiningsih and O. Troynikov. Force attenuation capacity and thermophysiological wear comfort of vertically lapped nonwoven fabric. The Journal of The Textile Institute, pages 1-9, 2017.

[50] F.J. Rey Martínez, M.A. Chicote, A.V. Peñalver, A.T. Gónzalez, and E.V. Gómez. Indoor air quality and thermal comfort evaluation in a spanish modern low-energy office with thermally activated building systems. Science and Technology for the Built Environment, 21(8):1091-1099, 2015.

[51] I. Oropeza-Perez, A.H. Petzold-Rodriguez, and C. Bonilla-Lopez. Adaptive thermal comfort in the main Mexican climate conditions with and without passive cooling. Energy and Buildings, 145:251-258, 2017.

[52] CEN EN. 15251-2007. Criteria for the indoor environment including thermal, indoor air quality, light and noise. Brussels: European Committee for Standardization, 2007.

[53] A. Matzarakis, H. Mayer, and M.G. Iziomon. Applications of a universal thermal index: physiological equivalent temperature. International journal of biometeorology, 43(2):76-84, 1999.

[54] I. Marincic, J.M. Ochoa, and M.G. Alpuche. Passive house for a desert climate. Eco-Architecture V: Harmonisation between Architecture and Nature, 142:13, 2014.

[55] I. Marincic, J.M. Ochoa, M.G. Alpuche, and G. Gómez-Azpeitia. Adaptive thermal comfort in warm dry climate: Economical dwellings in Mexico. In 26th Conference on Passive and Low Energy Architecture PLEA, pages 510$515,2009$.

[56] I. Oropeza-Perez and P.A. Østergaard. Potential of natural ventilation in temperate countries-a case study of denmark. Applied Energy, 114:520-530, 2014.

[57] L. Zhang, L. Zhang, and Y. Wang. Shape optimization of free-form buildings based on solar radiation gain and space efficiency using a multi-objective genetic algorithm in the severe cold zones of China. Solar Energy, 132:3850, 2016.

[58] I. Oropeza-Perez and P.A. Østergaard. Energy saving potential of utilizing natural ventilation under warm conditions-a case study of Mexico. Applied energy, 130:20-32, 2014. 
[59] M.K. Nematchoua, C.R. Raminosoa, R. Mamiharijaona, T. René, J.A. Orosa, W. Elvis, and P. Meukam. Study of the economical and optimum thermal insulation thickness for buildings in a wet and hot tropical climate: case of Cameroon. Renewable and Sustainable Energy Reviews, 50:1192-1202, 2015.

[60] J. Lei, J. Yang, and E.H. Yang. Energy performance of building envelopes integrated with phase change materials for cooling load reduction in tropical Singapore. Applied Energy, 162:207-217, 2016.

[61] C.W. Chen, C.W. Lee, and Y.W. Lin. Air conditioning-optimizing performance by reducing energy consumption. Energy \& Environment, 25(5):10191024, 2014.

[62] M. Sivak. Potential energy demand for cooling in the 50 largest metropolitan areas of the world: Implications for developing countries. Energy Policy, 37(4):1382-1384, 2009.

[63] J. Sousa. Energy simulation software for buildings: review and comparison. In International Workshop on Information Technology for Energy Applicatons-IT4Energy, Lisabon. Citeseer, 2012.

[64] S. Attia, J.L. Hensen, L. Beltrán, and A. De Herde. Selection criteria for building performance simulation tools: contrasting architects' and engineers' needs. Journal of Building Performance Simulation, 5(3):155-169, 2012.

[65] U. S. Department of Energy (DOE) EnergyPlus, Simulation Program v8. 0. https: / / energyplus . net, 11 de Mayo, 2017.

[66] University of Strathclyde ESP-r v11.11, Energy Systems Research Unit. http://www.esru.strath.ac.uk/programs/esp-r.htm, 22 de Mayo, 2017.

[67] University of Wisconsin-Madison TRNSYS v17, Solar Energy Laboratory. http://sel.me.wisc.edu/trnsys/, 10 de Noviembre, 2017.

[68] S. Attia and A. De Herde. Early design simulation tools for net zero energy buildings: a comparison of ten tools. In Conference Proceedings of 12th International Building Performance Simulation Association, 2011, 2011.

[69] D.B. Crawley, J.W. Hand, M. Kummert, and B.T. Griffith. Contrasting the capabilities of building energy performance simulation programs. Building and environment, 43(4):661-673, 2008.

[70] F. Westphal and R. Lamberts. Curso: Introducción a energyplus. Labeee, Florianópolis-SC, 2006. 
[71] D.B. Crawley, L.K. Lawrie, F.C. Winkelmann, W.F. Buhl, Y.J. Huang, C.O. Pedersen, R.K. Strand, R.J. Liesen, D.E. Fisher, M.J. Witte, and J. Glazer. EnergyPlus: creating a new-generation building energy simulation program. Energy and buildings, 33(4):319-331, 2001.

[72] P. Haugaard. Investigation and implementation of building simulation programmes-especially esp-r. DTU, Lyngby Denmark, pages 160-196, 2003.

[73] Welcome | TRNSYS: Transient Systems Simulation Tool Trnsys. http: / / www.trnsys.com/, 10 de Noviembre, 2017.

[74] F. Kuznik, J. Virgone, and K. Johannes. Development and validation of a new trnsys type for the simulation of external building walls containing pcm. Energy and Buildings, 42(7):1004-1009, 2010.

[75] S.A. Klein, W.A. Beckman, J.W. Mitchell, J.A. Duffie, N.A. Duffie, T.L. Freeman, J.C. Mitchell, J.E. Braun, B.L. Evans, J.P. Kummer, and R.E. Urban. TRNSYS 16-a transient system simulation program, user manual. Solar Energy Laboratory. Madison: University of Wisconsin-Madison, 2004.

[76] G. Salvalai, J. Pfafferott, and M.M. Sesana. Assessing energy and thermal comfort of different low-energy cooling concepts for non-residential buildings. Energy conversion and management, 76:332-341, 2013.

[77] M. Lebon, H. Fellouah, N. Galanis, A. Limane, and N. Guerfala. Numerical analysis and field measurements of the airflow patterns and thermal comfort in an indoor swimming pool: a case study. Energy Efficiency, 10(3):527-548, 2017.

[78] S. Zhang, Y. Jiang, W. Xu, H. Li, and Z. Yu. Operating performance in cooling mode of a ground source heat pump of a nearly-zero energy building in the cold region of china. Renewable Energy, 87:1045-1052, 2016.

[79] I. Beausoleil-Morrison, F. Macdonald, M. Kummert, T. McDowell, R. Jost, and A. Ferguson. The design of an ESP-r and TRNSYS co-simulator. In Proceedings of the International IBPSA Conference, Sydney, Australia, 2011.

[80] Ó.R. Rivera and Fundación Laboral de la Construcción. Manual práctico de cálculos térmicos de edificios. Oficios. Eficiencia energética. Tornapunta, 2013.

[81] Serrano, P. https://www.certificadosenergeticos.com/, 03 de Septiembre de 2018.

[82] A. Saltelli, S. Tarantola, F. Campolongo, and M. Ratto. Sensitivity analysis in practice: a guide to assessing scientific models. John Wiley \& Sons, 2004. 
[83] G.C. Rodríguez, A.C. Andrés, F.D. Muñoz, J.M.C. López, and Y. Zhang. Uncertainties and sensitivity analysis in building energy simulation using macroparameters. Energy and Buildings, 67:79-87, 2013.

[84] P. Antoniadou and A.M. Papadopoulos. Occupants' thermal comfort: State of the art and the prospects of personalized assessment in office buildings. Energy and Buildings, 2017.

[85] W. Tian. A review of sensitivity analysis methods in building energy analysis. Renewable and Sustainable Energy Reviews, 20:411-419, 2013.

[86] K.J. Lomas and H. Eppel. Sensitivity analysis techniques for building thermal simulation programs. Energy and buildings, 19(1):21-44, 1992.

[87] A. Ioannou and L.C.M. Itard. Energy performance and comfort in residential buildings: Sensitivity for building parameters and occupancy. Energy and Buildings, 92:216-233, 2015.

[88] M. Basinska, H. Koczyk, and E. Szczechowiak. Sensitivity analysis in determining the optimum energy for residential buildings in Polish conditions. Energy and Buildings, 107:307-318, 2015.

[89] H. Breesch and A. Janssens. Performance evaluation of passive cooling in office buildings based on uncertainty and sensitivity analysis. Solar energy, 84(8):1453-1467, 2010.

[90] R. Ruiz Flores, S. Bertagnolio, and V. Lemort. Global sensitivity analysis applied to total energy use in buildings. In Proceedings of the 2 nd International High Performance Buildings Conference, 2012.

[91] M.C. Peel, B.L. Finlayson, and T.A. McMahon. Updated world map of the Köppen-Geiger climate classification. Hydrology and earth system sciences discussions, 4(2):439-473, 2007.

[92] E. Balvís, Ó. Sampedro, S. Zaragoza, A. Paredes, and H. Michinel. A simple model for automatic analysis and diagnosis of environmental thermal comfort in energy efficient buildings. Applied Energy, 177:60-70, 2016.

[93] J. Remund. Meteonorm: Irradiation data for every place on earth. Bern2014, 2014.

[94] T.L. Madsen. Description of thermal manikin for measuring thermal insulation values of clothing. Thermal Insulation Report, (48), 1976.

[95] E. DIN. 13779 (2007) ventilation for non-residential buildings-performance requirements for ventilation and room-conditioning systems. British Standard, 2007. 
[96] F. Ascione, N. Bianco, C. De Stasio, G.M. Mauro, and G.P. Vanoli. Multistage and multi-objective optimization for energy retrofitting a developed hospital reference building: A new approach to assess cost-optimality. Applied Energy, 174:37-68, 2016.

[97] T.M. Echenagucia, A. Capozzoli, Y. Cascone, and M. Sassone. The early design stage of a building envelope: Multi-objective search through heating, cooling and lighting energy performance analysis. Applied Energy, 154:577$591,2015$.

[98] J.C. Lam and S.C. Hui. Sensitivity analysis of energy performance of office buildings. Building and Environment, 31(1):27-39, 1996.

[99] R. Carrasco, P. Fernández de Córdoba, L.M. García-Raffi, and J.M. Sanchís. Métodos de simulación Monte Carlo y sus aplicaciones. Universidad Politécnica de Valencia, 2000.

[100] Universidad de Barcelona. https:www.ub.edu/stat/ Grups Innovacio/Statmedia/demo/Temas/Capitulo4/ B0C4m1t1. htm, 21 de Junio de 2018.

[101] D.G. Altman. Practical statistics for medical research. CRC press, 1990.

[102] W.W. Daniel and L. Hernández. Bioestadísticabase para el análisis de las ciencias de la salud. Number 574.015195 D3/2005. 2005.

[103] R.C. Elston and W.D. Johnson. Essentials of biostatistics. 1987.

[104] S. Pértegas Díaz and S. Pita Fernández. La distribución normal. Cad Aten Primaria, 8:268-274, 2001.

[105] R. Robledo-Fava, M.C Hernández-Luna, P. Fernández de Córdoba, H. Michinel, and S. Zaragoza. Analysis of the sensitivity of bim-based numerical simulations to variations in subjective human parameters. En preparacion. 



\section{Anexo I: Estudio de caso: edificio en República Dominicana}

\section{Caso de estudio}

En el presente caso de estudio, se analizó el comportamiento energético de un edificio localizado dentro de las instalaciones de la Universidad Autónoma de Santo Domingo (UASD), que cuenta con una cafetería la cuál será nuestro objetivo para simular y analizar matemáticamente; con ello, obtendremos resultados que van enfocados hacia obtener un menor consumo energético así como a verificar lo obtenido en esta tesis de doctorado.

La universidad está situada en Santo Domingo, capital de República Dominicana, (latitud $18^{\circ} 27^{\prime} 42.73^{\prime \prime} \mathrm{N}$, longitud $69^{\circ} 54^{\prime} 52.93^{\prime \prime}$ ), a una altura de $18 \mathrm{~m}$ sobre el nivel del mar (Fig. 1).

El clima que prevalece en Santo Domingo es tropical; la mayoría de los meses del año están marcados por lluvias significativas. La corta estación seca tiene poco impacto. Este clima es considerado Am (A - tropical, m - monzónico), según la clasificación climática de Köppen-Geiger. La temperatura media anual es $25.7^{\circ} \mathrm{C}$, con precipitaciones promedio de $1661 \mathrm{~mm}$.

El edificio tiene las siguientes orientaciones para los 4 cerramientos verticales: hacia el norte colinda con la avenida Paulo III, hacia el suroeste con la calle Cristóbal de Llenares, hacia el este con la avenida Santo Tomás de Aquino y hacia el sureste con la avenida Dr. Bernardo Correa y Cidrón (Fig. 1 y Tabla 1).

\section{Estructura y uso del caso de estudio}

El área a estudiar $\left(122.04 \mathrm{~m}^{2}\right)$, se utiliza como cafetería y se encuentra situada en la planta baja del Edificio de los Gremios Sindicales, conformado por un sistema de pórticos y muros de hormigón armado (Sistema Dual). En las siguientes imágenes de la Fig. 2 podemos observar características de su estructura exterior e interior. 

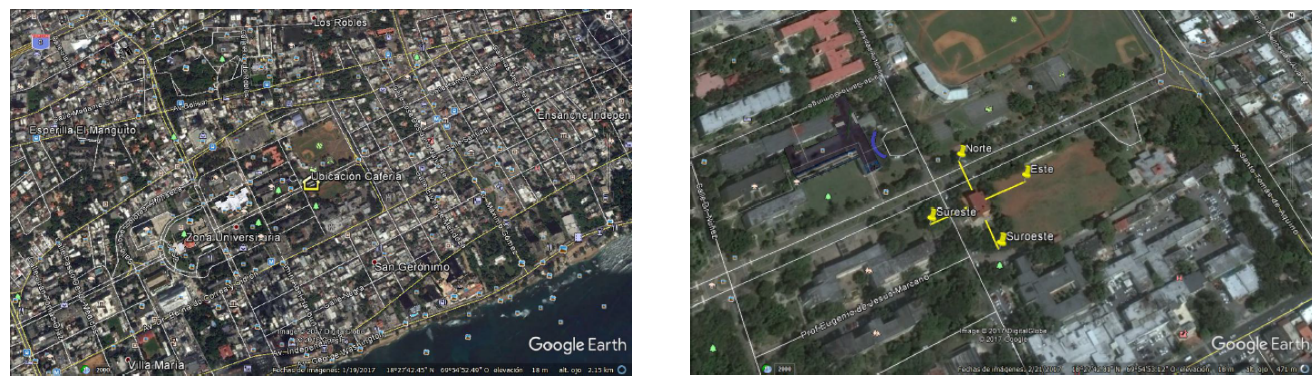

Figura 1: Ubicación y orientaciones del caso de estudio (República Dominicana).

\begin{tabular}{|c|c|c|c|}
\hline Orientación & Abreviatura & Categoría & Ángulo \\
\hline \hline Norte & $\mathrm{N}$ & Elevación posterior & $337.23^{0}$ \\
\hline Sureste & SE & Elevación frontal & $155.97^{0}$ \\
\hline Este & $\mathrm{E}$ & Lateral derecho & $64.81^{0}$ \\
\hline Suroeste & SO & Lateral izquierdo & $247.21^{0}$ \\
\hline
\end{tabular}

Tabla 1: Orientaciones de la fachada.
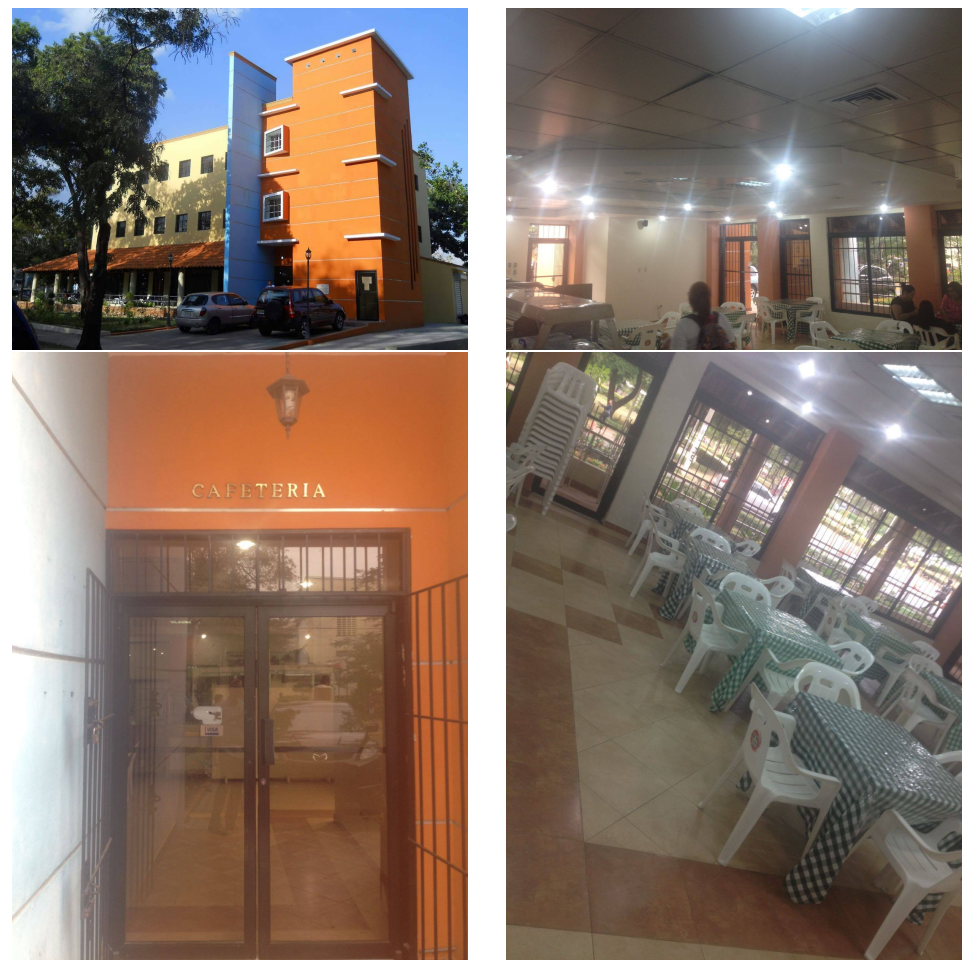

Figura 2: Imágenes reales del edificio y cafetería en República Dominicana. 
La cantidad de personas que asisten en horas pico a la cafetería es aproximadamente de 80 personas, entre empleados y clientes. En la figura 3, se muestra el plano de la cafetería que ha sido dividido en cuantro zonas a estudiar: comedor, cocina, cuarto frío y almacén o depósito.

- Comedor, en esta zona se encuentra lo relacionado con el consumo de alimentos, así como los sanitarios.

- Cocina, en ella se preparan los alimentos de la cafetería.

- Depósito, este lugar se utiliza para el almacenamiento de todo lo relacionado con la cafetería que puede mantenerse a temperatura ambiente.

- Cuarto frío, el propósito de esta zona es mantener los productos que deben ser refrigerados.

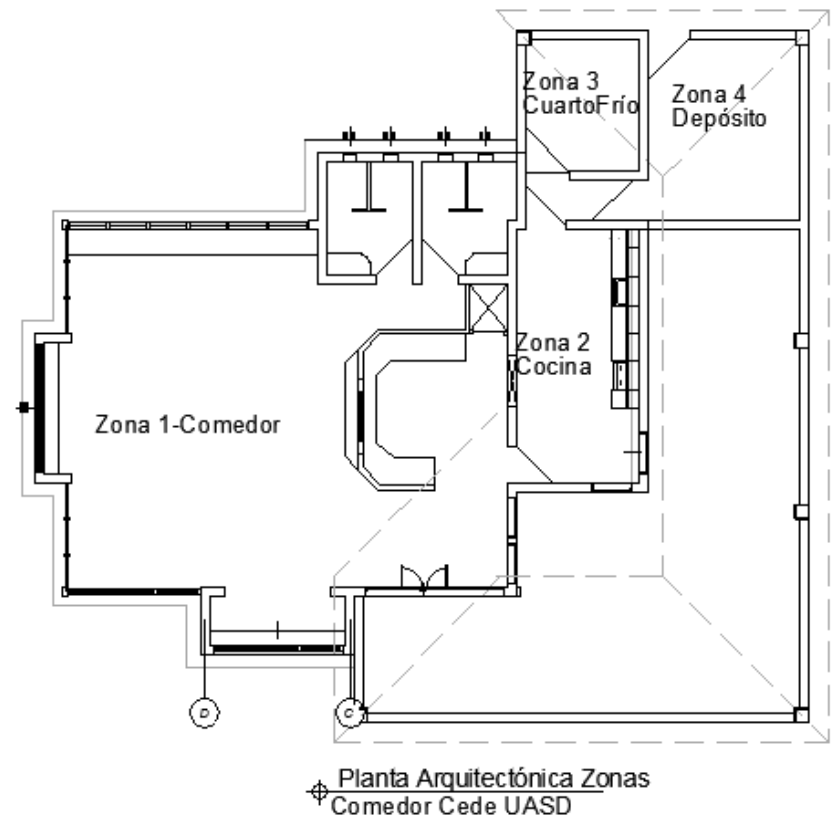

Figura 3: Geometría del modelo numérico en el software de diseño gráfico SketchUp.

En la Tabla 2 se muestran los valores de áreas y volúmenes para las zonas descritas previamente.

\section{Definición del modelo en TRNBuild}

La introducción de la cafetería del edificio en el software TRNSYS se realizó de la siguiente manera: el primer paso fue la implementación de las zonas y todos sus 


\begin{tabular}{|c|c|c|}
\hline Zonas & Área $\left.\mathbf{~ m}^{\mathbf{2}}\right)$ & Volumen $\left(\mathbf{m}^{\mathbf{3}}\right)$ \\
\hline \hline Comedor & 86.11 & 232.497 \\
\hline Cocina & 15.20 & 41.04 \\
\hline Depósito & 13.38 & 36.126 \\
\hline Cuarto frío & 7.35 & 19.845 \\
\hline
\end{tabular}

Tabla 2: Área y volumen para las zonas del caso de estudio.

elementos como ubicación, orientaciones, muros, capas de materiales, ganancias, ventilación, infiltración, horarios y confort en TRNBuild. El segundo paso corresponde a ingresar en los modelos TRNSYS datos como clima ; entradas como el edificio descrito anteriormente para obtener salidas que mostrarán a través de gráficos el rendimiento térmico y las demandas de energía de los edificios. El proceso de definición de los edificios en TRNBuild, incluye la siguiente información:

\section{- Estructura del edificio}

Los cerramientos y los materiales utilizados para definir el caso de estudio se detallan en la Tabla 3.

\begin{tabular}{|c|c|c|c|c|}
\hline Caso de estudio & Tipo de muro & Capas de materiales & $\kappa$ & $\rho$ \\
\hline \hline \multirow{5}{*}{ Rep. Dominicana } & \multirow{4}{*}{$\begin{array}{c}\text { Cerramiento } \\
\text { externo } \\
\text { principal }\end{array}$} & Cemento y arena & 3.60 & 1800 \\
\cline { 3 - 5 } & \multirow{3}{*}{ Suelo } & Bloque de concreto & 1.84 & 1400 \\
\cline { 3 - 5 } & & Cemento y arena & 3.60 & 1800 \\
\cline { 3 - 5 } & \multirow{3}{*}{ Techo } & Arena y grava & 7.20 & 2000 \\
\cline { 3 - 5 } & & Yeso y enlucidos & 3.60 & 1800 \\
\cline { 3 - 5 } & & Yeso y enlucidos & 3.60 & 1800 \\
\cline { 3 - 5 } & & Hormigón armado & 9.0 & 2400 \\
\cline { 3 - 5 } & & Yeso y enlucidos & 3.60 & 1800 \\
\hline
\end{tabular}

Tabla 3: Propiedades térmicas y estructurales.

Las características de las ventanas se describen en la (Tabla 4). Se han definido 2 tipos de ventana para este caso de estudio. Entre sus principales características se encuentran: el porcentaje de marco, la transmitancia $(U)$ y la ganancia solar $(G-$ Value $)$. 


\begin{tabular}{|c|c|c|c|c|c|c|}
\hline \multirow{2}{*}{ Caso de estudio } & \multirow{2}{*}{ Tipo } & \multirow{2}{*}{ Tipo de vidrio } & Espesor & $\%$ & $U$ & $G-$ Value \\
\cline { 4 - 6 } & & & $(\mathrm{mm})$ & Marco & $\left(\mathrm{W} / \mathrm{m}^{2} \mathrm{~K}\right)$ & $\left(\mathrm{kg} / \mathrm{m}^{3}\right)$ \\
\hline \hline \multirow{2}{*}{ Rep. Dominicana } & Ventana01 & Simple claro & 4 & 15 & 5.8 & 0.862 \\
\cline { 2 - 7 } & Ventana02 & Simple claro gris & 4 & 15 & 5.73 & 0.580 \\
\hline
\end{tabular}

Tabla 4: Propiedades térmicas y estructurales en ventanas.

La Tabla 5 muestra la definición de los cerramientos para cada una de las zonas de acuerdo a los materiales detallados anteriormente en la Tabla 3.

\begin{tabular}{|c|c|c|c|c|}
\hline Caso de estudio & Zona & Tipo de muro & Área $\left(m^{2}\right)$ & Orientación \\
\hline \multirow{27}{*}{ Rep. Dominicana } & \multirow{6}{*}{ Cocina } & Principal & 7.86 & Norte \\
\hline & & Principal & 15.66 & Suroeste \\
\hline & & Principal & 15.60 & - \\
\hline & & Principal & 7.86 & - \\
\hline & & Suelo & 15.20 & Horizontal \\
\hline & & Forjado & 15.20 & Horizontal \\
\hline & \multirow{9}{*}{ Comedor } & Principal & 28.22 & Norte \\
\hline & & Principal & 6.21 & Suroeste \\
\hline & & Principal & 15.60 & - \\
\hline & & Principal & 3.32 & - \\
\hline & & Principal & 0.96 & - \\
\hline & & Principal & 29 & Suroeste \\
\hline & & Principal & 29.85 & Este \\
\hline & & Suelo & 86.11 & Horizontal \\
\hline & & Forjado & 86.11 & Horizontal \\
\hline & \multirow{6}{*}{ Depósito } & Principal & 9.29 & Norte \\
\hline & & Principal & 11.34 & Suroeste \\
\hline & & Principal & 9.56 & Sureste \\
\hline & & Principal & 15.74 & - \\
\hline & & Suelo & 13.38 & Horizontal \\
\hline & & Forjado & 13.38 & Horizontal \\
\hline & \multirow{6}{*}{ Cuarto Frío } & Principal & 7.38 & Suroeste \\
\hline & & Principal & 7.29 & Este \\
\hline & & Principal & 15.74 & - \\
\hline & & Principal & 0.96 & - \\
\hline & & Suelo & 7.35 & Horizontal \\
\hline & & Forjado & 7.35 & Horizontal \\
\hline
\end{tabular}

Tabla 5: Estructura de la fachada de la cocina. 


\section{- Ubicación y orientaciones}

Esta información fue descrita al inicio de la descripción del caso de estudio.

\section{- Definición de zonas}

Las zonas descritas en la Tablas 2 y 5, se crearon como comedor, cocina, depósito y cuarto frío.

\section{- Horarios}

En la Tabla 6 se muestran los diferentes tipos de horarios creados para este caso de estudio; para empleados y clientes establecemos un valor mayor que 0 y menor o igual a 1 en los períodos donde hay actividad en el edificio, en cualquier otro caso, el valor es 0 .

Esta cafetería cuenta con una capacidad media de 70 clientes y se encuentran laborando 11 empleados, para un promedio de 81 personas; el porcentaje de personas a distintos períodos de tiempo cambia, ya que existen horas en las cuales los clientes varían en mayor o menor proporción dependiendo si es horario de almuerzo o merienda, en la Tabla 8 se muestra la capacidad aproximada para distintos horarios.

\begin{tabular}{|c|c|c|}
\hline Días & Empleado (Ent/Sal) & Cliente (Ent/Sal) \\
\hline \hline Lunes a Sábado & 07:00 a 18:00 & $8: 00$ a 17:00 \\
\hline Domingo & 07:00 a 16:00 & $8: 00$ a 15:00 \\
\hline
\end{tabular}

Tabla 6: Horario.

\begin{tabular}{|c|c|}
\hline 0 & Si no hay personas en el edificio \\
\hline \hline 1 & Si hay personas en el edificio \\
\hline \hline$[0,1]$ & \% de ocupación \\
\hline \hline
\end{tabular}

Tabla 7: Escala de porcentaje.

\section{- Infiltraciones}

El valor de infiltración se ha establecido por defecto en TRNSYS en 0.6 renovaciones $/ h$. 


\begin{tabular}{|c|c|c|}
\hline \multicolumn{3}{|c|}{ Lunes a sábado (clientes y empleados) } \\
\hline \hline Desde & Hasta & Valor \\
\hline \hline $00: 00$ & $7: 00$ & 0 \\
\hline $7: 00$ & $8: 00$ & 0.13 \\
\hline $8: 00$ & $11: 00$ & 0.56 \\
\hline $11: 00$ & $12: 30$ & 0.75 \\
\hline $13: 00$ & $14: 00$ & 1 \\
\hline $14: 00$ & $15: 00$ & 0.44 \\
\hline $15: 00$ & $17: 00$ & 0.32 \\
\hline $17: 00$ & $18: 00$ & 0.13 \\
\hline $18: 00$ & $24: 00$ & 0 \\
\hline \multicolumn{3}{|c|}{ Domingo } \\
\hline
\end{tabular}

\begin{tabular}{|c|c|c|}
\hline \hline $00: 00$ & $7: 00$ & 0 \\
\hline $07: 00$ & $8: 00$ & 0.07 \\
\hline $08: 00$ & $15: 00$ & 0.35 \\
\hline $15: 00$ & $16: 00$ & 0.07 \\
\hline $18: 00$ & $24: 00$ & 0 \\
\hline \multicolumn{2}{|c|}{ Equipos Hora Pico } \\
\hline
\end{tabular}

\begin{tabular}{|c|c|c|}
\hline \hline $00: 00$ & $11: 30$ & 0 \\
\hline $11: 30$ & $12: 30$ & 1 \\
\hline $12: 30$ & $13: 30$ & 0.5 \\
\hline $13: 30$ & $24: 00$ & 0 \\
\hline \multicolumn{3}{|c|}{ Estufas } \\
\hline
\end{tabular}

\begin{tabular}{|c|c|c|}
\hline \hline $00: 00$ & $07: 30$ & 0 \\
\hline $07: 30$ & $13: 30$ & 1 \\
\hline $01: 30$ & $24: 00$ & 0 \\
\hline \multicolumn{3}{|c|}{ Luminarias } \\
\hline \hline
\end{tabular}

\begin{tabular}{|c|c|c|}
\hline \hline $00: 00$ & $07: 30$ & 0 \\
\hline $07: 30$ & $13: 30$ & 1 \\
\hline $13: 30$ & $24: 00$ & 0 \\
\hline \multicolumn{3}{|c|}{ Fin de semana } \\
\hline \hline $00: 00$ & $24: 00$ & 0 \\
\hline
\end{tabular}

Tabla 8: Distintos horarios de uso para el caso de República Dominicana. 


\begin{tabular}{|c|c|}
\hline \multicolumn{2}{|c|}{ Semana } \\
\hline \hline Días & Nombres \\
\hline \hline Lunes a sábado & Horario clientes y empleados \\
\hline Domingo & Horario Domingo \\
\hline
\end{tabular}

Tabla 9: Horario semanal.

\section{- Ventilación}

Se estimó un valor para cada zona (ver Tabla 10), en base a las normativas previamente utilizadas para los primeros casos de estudio.

\begin{tabular}{|c|c|c|}
\hline Zona & Personas & Caudal mínimo $\left(\right.$ pers $\left.\cdot \mathbf{d m}^{\mathbf{3}} / \mathbf{s}\right)$ \\
\hline \hline Comedor & 77 & 616 \\
\hline Cocina & 4 & 32 \\
\hline
\end{tabular}

Tabla 10: Caudal mínimo de ventilación para zona comedor y cocina.

\section{- Ganancias internas}

Las ganancias internas de los edificios son de las propiedades más importantes para introducir en el análisis del comportamiento térmico. Esta información se muestra en las Tabla 11.

\begin{tabular}{|c|c|c|}
\hline Equipo & Cantidad & Potencia $(W)$ \\
\hline \hline Tostadora & 1 & 1553 \\
\hline Cafetera & 1 & 1500 \\
\hline Congelador & 1 & 1408 \\
\hline Refrigerador & 3 & 1056 \\
\hline Microondas & 2 & 2404 \\
\hline Estufa Indust. & 1 & 25614 \\
\hline Congelador & 1 & 3520 \\
\hline Horno Eléctri. & 1 & 1964 \\
\hline
\end{tabular}

Tabla 11: Datos de entrada de ganancias internas.

\begin{tabular}{|c|c|c|c|}
\hline Actividad realizada & Calor total $(W)$ & Calor sensible $(W)$ & Calor latente $(W)$ \\
\hline \hline Servir y comer & 170 & 75 & 95 \\
\hline
\end{tabular}

Tabla 12: Actividad realizada por los ocupantes en el comedor. 


\begin{tabular}{|c|c|c|c|}
\hline Actividad realizada & Calor total $(W)$ & Calor sensible $(W)$ & Calor latente $(W)$ \\
\hline \hline Trabajo ligero & 185 & 90 & 95 \\
\hline
\end{tabular}

Tabla 13: Actividad realizada por los ocupantes en la cocina.

\section{- Confort}

El confort térmico es uno de los principales factores relacionado directamente con el comportamiento térmico del edificio, por esta razón, los valores de factor de vestimenta, tasa metabólica, velocidad relativa del aire se introdujeron en TRNSYS (basados en la normativa ISO 7730). La Tabla 4.5 muestra los valores para el actual caso de estudio.

\begin{tabular}{|c|l|c|}
\hline \multicolumn{3}{|c|}{ Confort cocina } \\
\hline \hline Parámetros & Valores & Características \\
\hline \hline Factor vestimenta & $\begin{array}{l}\text { Ropa ligera de verano (Pantalo- } \\
\text { nes largos ligeros, camisa de cue- } \\
\text { llo abierto con mangas cortas) }\end{array}$ & 0.5 clo \\
\hline Factor metabólico & $\begin{array}{l}\text { De pie, trabajo moderado (Activi- } \\
\text { dad de venta, trabajo doméstico, } \\
\text { funcionamiento de una máquina) }\end{array}$ & 2.0 met \\
\hline Trabajo externo & Valor por defecto & 0 \\
\hline Velocidad relativo al aire & Casi siempre cero & 0.1 \\
\hline
\end{tabular}

Tabla 14: Valores de confort para la cocina.

\begin{tabular}{|c|l|c|}
\hline \multicolumn{3}{|c|}{ Confort comedor } \\
\hline \hline Parámetros & Valores & Características5 \\
\hline \hline Factor de vestimenta & $\begin{array}{l}\text { Ropa ligera de verano (panta- } \\
\text { lones largos ligeros, camisa de } \\
\text { cuello abierto con mangas cor- } \\
\text { tas) }\end{array}$ & 0.5 clo \\
\hline Factor metabólico & $\begin{array}{l}\text { Asiento, trabajo ligero (oficina, } \\
\text { hogar, escuela, laboratorio) }\end{array}$ & $1.2 \mathrm{met}$ \\
\hline Trabajo externo & Valor por defecto & 0 met \\
\hline Velocidad del aire relativo & Casi siempre cero & $0.1 \mathrm{~m} / \mathrm{s}$ \\
\hline
\end{tabular}

Tabla 15: Datos de entrada de confort para comedor. 


\section{Resultados}

En esta sección presentaremos y analizaremos los resultados que obtuvimos de la simulación de la cocina en TRNSYS. Presentaremos una serie de gráficas analizadas con diferentes intervalos de tiempo con la finalidad de obtener resultados más precisos y canalizar mejor el flujo de información de estas gráficas.

\section{Temperaturas anuales}

Las primeras gráficas corresponden a períodos anuales de simulación de las temperaturas de las 4 zonas que se introdujeron previamente a TRNSYS.

\section{- Temperatura ambiente}

En la figura 4, se observan las temperaturas ambiente de todo un año de simulación obtenidos de la estación climatológica de Bayaguana; los valores de las temperaturas varían entre $17^{\circ} \mathrm{C}$ y $35^{\circ} \mathrm{C}$; este tipo de temperaturas son consideradas normales por los habitantes de ese lugar. Como se observa en la gráfica, los meses más fríos corresponden a los meses de diciembre a abril; por el contrario, el resto del año corresponde a los meses de verano con las temperaturas más altas.

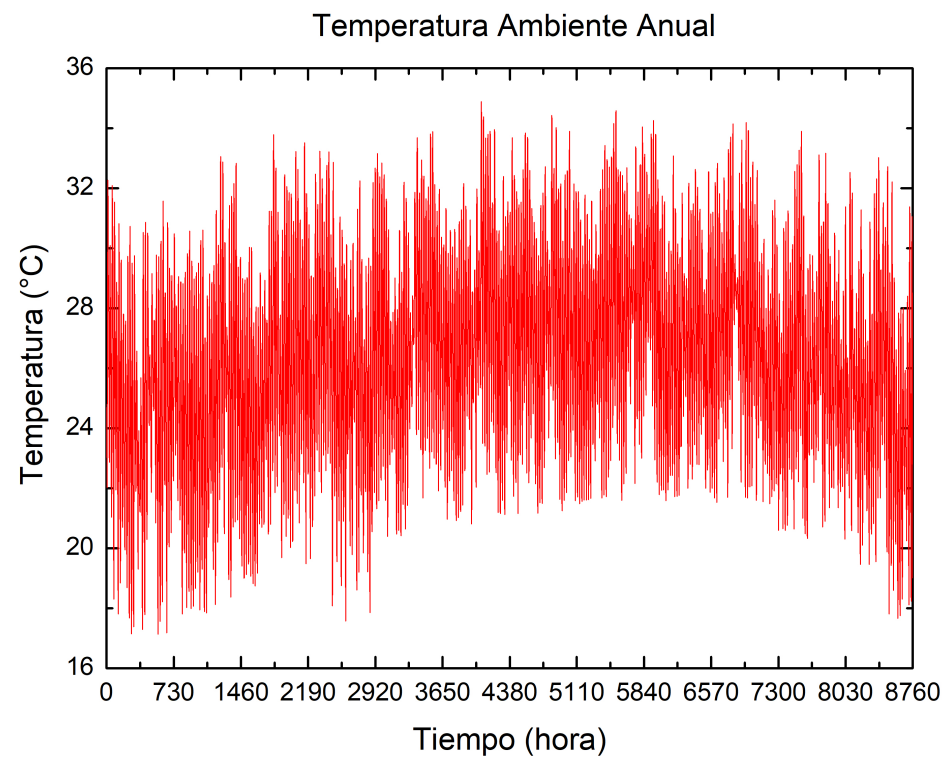

Figura 4: Temperatura anual: ambiente. 


\section{- Zona: Comedor}

Como se observa en la figura 5, la zona del comedor tiene una mayor variación de temperatura; esto se debe principalmente al intercambio constante de personas desde la zona exterior o entre las demás zonas hacia el comedor. Además, dicha zona no dispone de un buen sistema de ventilación, por lo tanto, podemos observar que las temperaturas varían desde $\operatorname{los} 21^{\circ} \mathrm{C}$ hasta $40^{\circ} \mathrm{C}$.

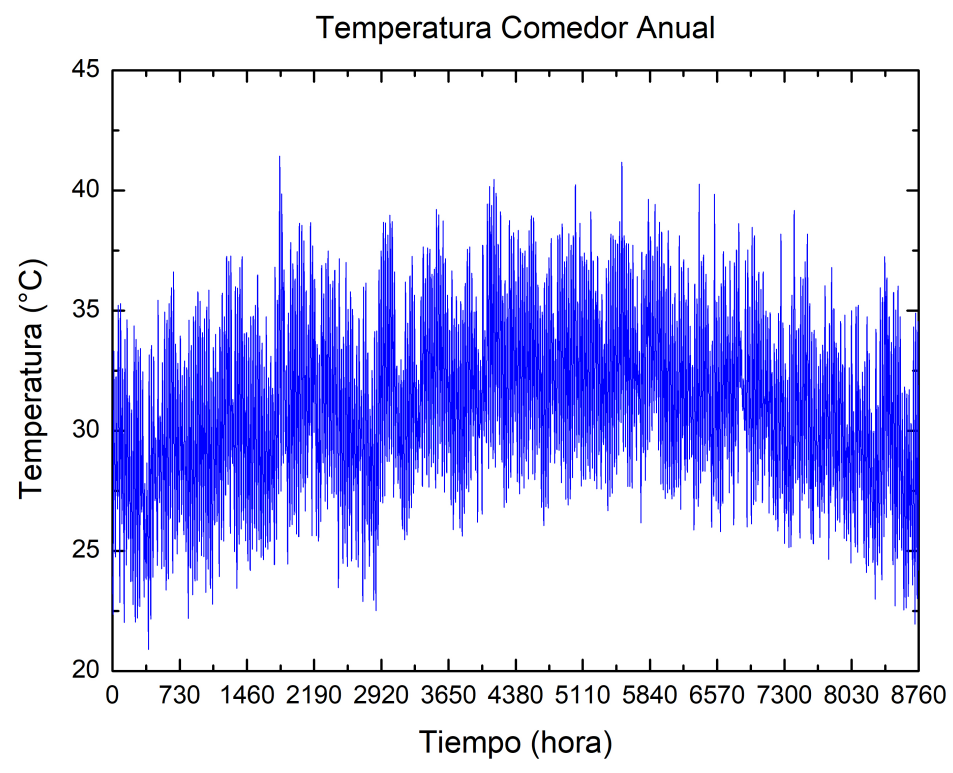

Figura 5: Temperatura anual: zona del comedor.

\section{- Zona: Cocina}

Sin duda alguna, y corroborado con los valores de las temperaturas que se observan en la figura 6, una de las zonas con mayor temperatura es la cocina. Podemos observar que las altas temperaturas se mantienen durante todo el año, debido al gran desprendimiento de calor de los equipos eléctricos instalados (estufa industrial, horno eléctrico, etc.) y a las actividades que se realizan en la zona. Además, las altas temperaturas también se deben a la poca ventilación que tiene la zona. 


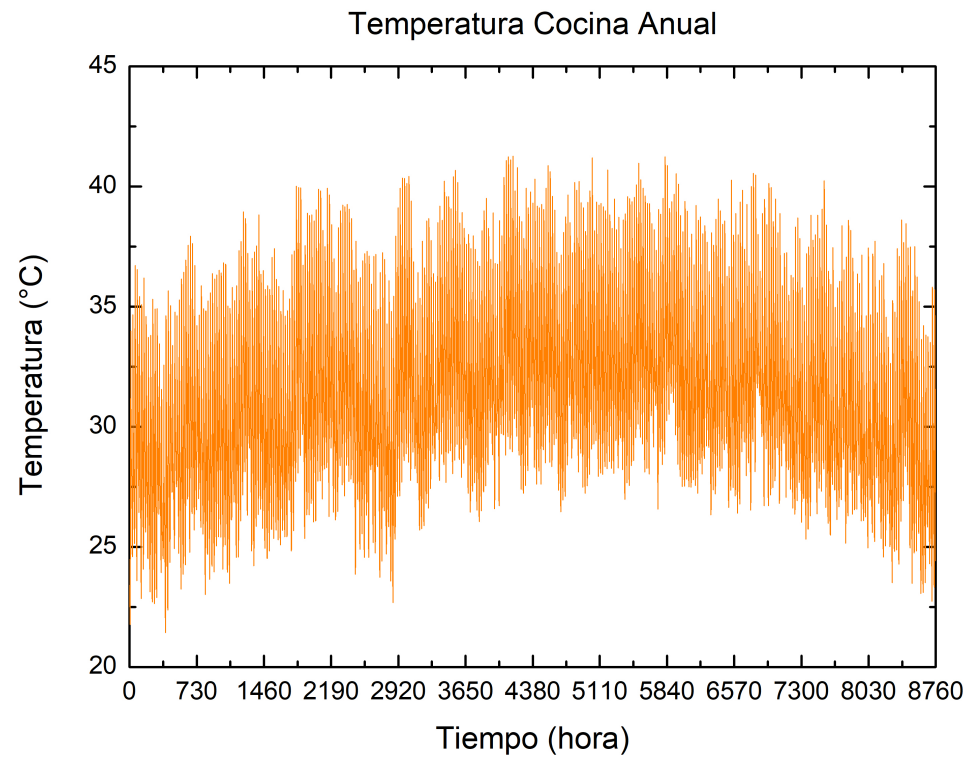

Figura 6: Temperatura anual: zona de la cocina.

\section{- Zona: Depósito}

La Fig. 7, corresponde a la zona del depósito, donde las temperaturas obtenidas dependen pricipalmente de las zonas adyacentes a ella; en este caso, la cocina, cuarto frío y el exterior. Se debe destacar que la temperatura del aire de los alimentos almacenados en este lugar es muy importante, ya que se deben de mantener en buen estado y con ello, garantizar la calidad de los mismos; se debería considerar dar una mayor ventilación a dicha zona.

\section{- Zona: Cuarto frío}

Para terminar con los resultados anuales de las zonas, tenemos la gráfica que corresponde al cuarto frío y, precisamente, podemos observar como se mantiene la temperatura constante todo el año como se observa en la Fig. 8. Por tal razón, no va a sufrir ningún cambio excepto que exista algún problema o se desconecte para dar mantenimiento.

\section{Temperaturas mensuales}

A continuación, analizaremos el comportamiento de la temperatura de las 4 zonas por un período mensual. Después de realizar algunas simulaciones, se ha decidido 


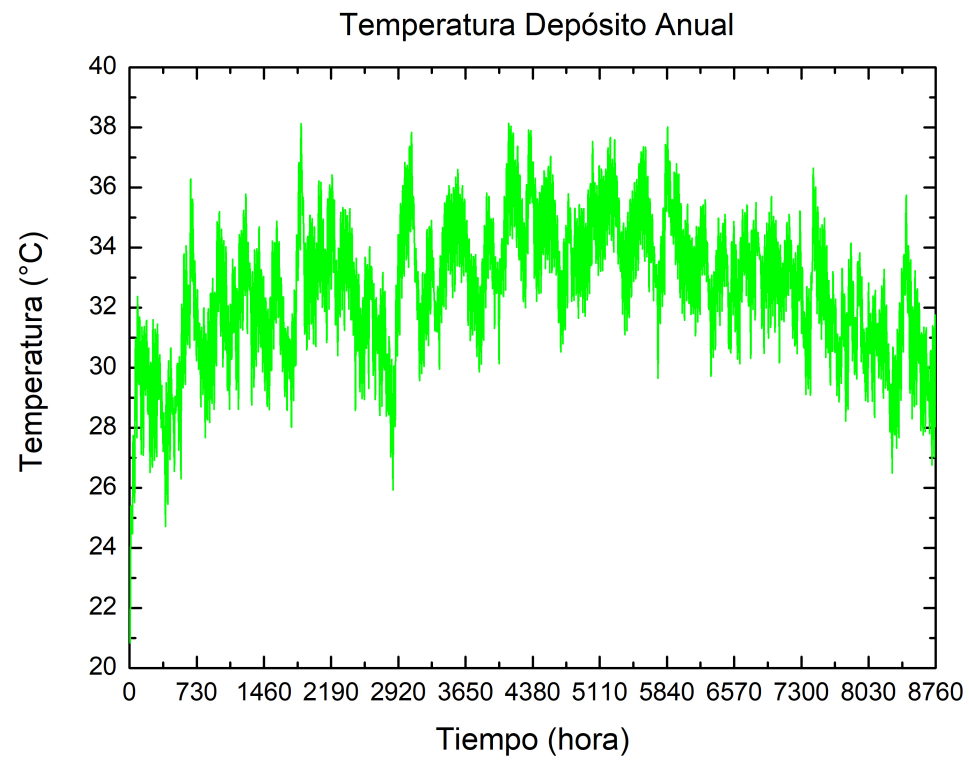

Figura 7: Temperatura anual: zona del depósito.

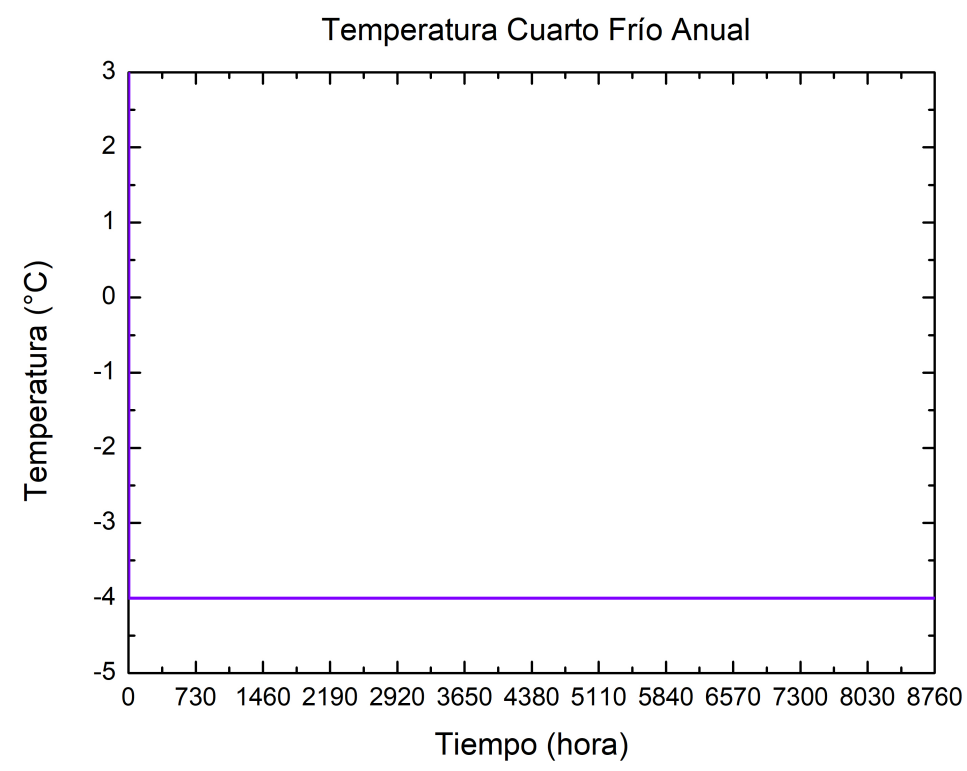

Figura 8: Temperatura anual: zona del cuarto frío. 
presentar los resultados de los meses de enero y agosto debido a que forman parte de dos estaciones con temperaturas muy diferentes, en este caso, invierno y verano.

\section{- Mes: enero}

La Fig. 9, muestra las temperaturas correspondientes al mes de enero de las 4 zonas: comedor (azul), cocina (naranja), cuarto frío (morada) y depósito (verde). Podemos observar que la zona del comedor y la cocina son las que tienen las temperaturas más altas, alcanzando $\operatorname{los} 40^{\circ} \mathrm{C}$. En cambio, la zona del depósito, varía entre los $22^{\circ} \mathrm{C} \mathrm{y} 33^{\circ} \mathrm{C}$ aproximadamente. Por último, tenemos la temperatura constante del cuarto frío, $-4^{\circ} \mathrm{C}$.

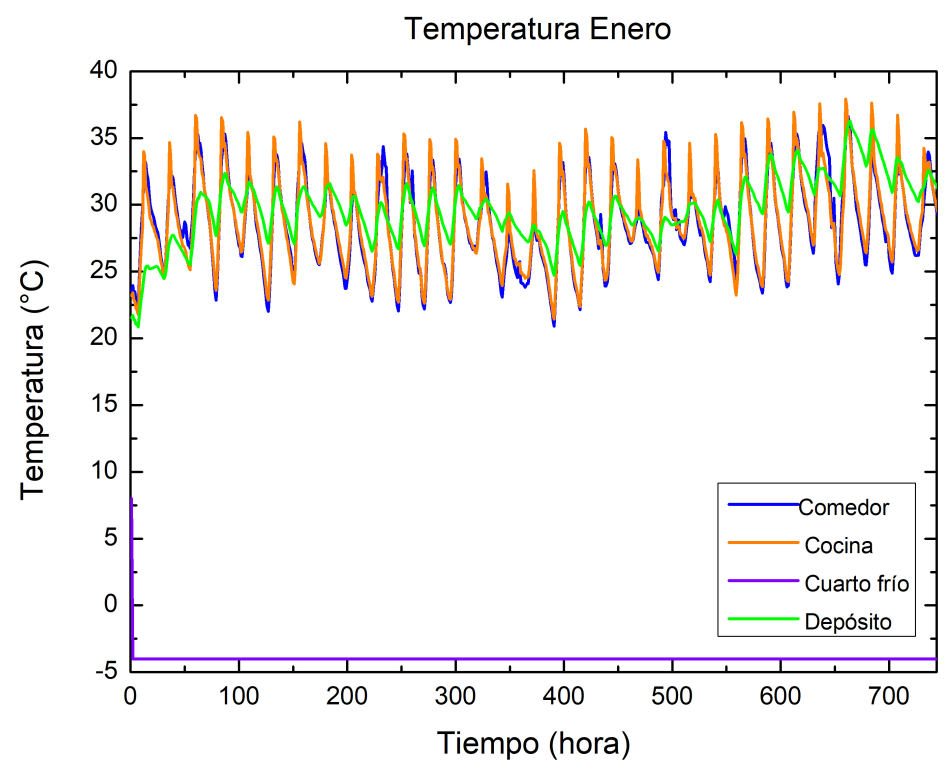

Figura 9: Temperatura del mes de enero.

\section{- Mes: agosto}

Para uno de los meses de verano, Fig. 10, se observa que hay un aumento de temperatura en las 3 zonas manteniendo la zona del cuarto frío constante $\left(-4^{\circ} \mathrm{C}\right)$; esto es, que el mes de agosto tiene las temperaturas más altas durante todo el año. Analizando la gráfica de la figura 10, podemos observar que, tanto en la cocina como en el comedor, la temperatura varía desde $27^{\circ} \mathrm{C}$ hasta $41^{\circ} \mathrm{C}$, mientras que la temperatura del depósito varía entre $32^{\circ} \mathrm{C}$ a $35^{\circ} \mathrm{C}$. 


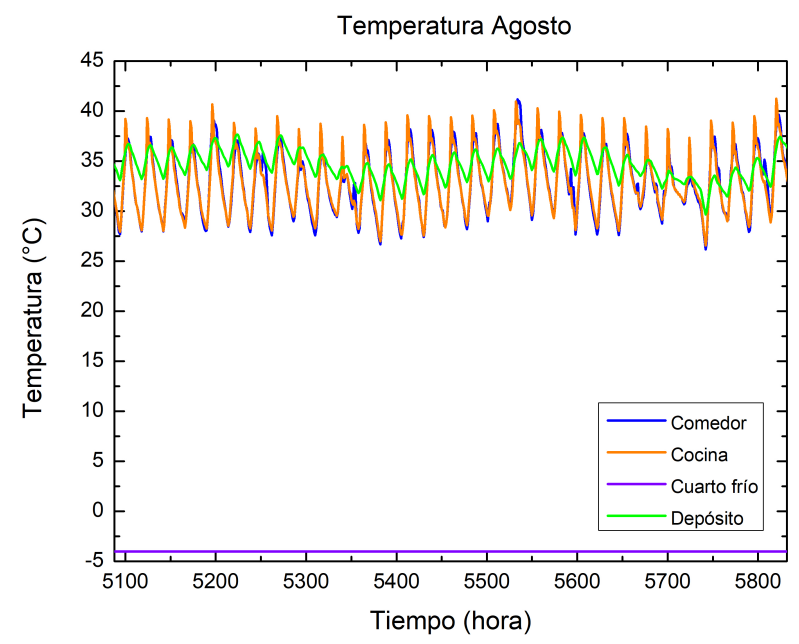

Figura 10: Temperatura del mes de agosto.

\section{Resultados y conclusiones de las demandas de energía}

En la tabla 16 se presentan los cambios en la demanda de energía debido a las variaciones en los criterios del diseñador en climas como el caso de Rep. Dom. donde prácticamente todo el año la temperatura es elevada. Como ejemplo, y por completitud, presentamos las variaciones en la demanda energética acumulada durante el mes de enero del recinto analizado (cafetería). Hemos fijado una temperatura de consigna de $25^{\circ} \mathrm{C}$ y dos valores del factor de vestimenta $(0.3$ clo y 0.6 clo) y dos valores del factor metabólico (1.2 met y 1.6 met). Si bien, en este caso, el impacto energético de la elección de clo y met por parte del diseñador es pequeño (debemos tener en cuenta que hemos considerado sólo el mes de enero) también se contrasta que el impacto energético de la variación del factor metabólico (3.5\%) es superior a lo relacionado con el factor de vestimenta $(0.8 \%)$. Hemos elegido analizar estos ensayos en el mes de enero para estudiar uno de los meses mas fríos y con menor demanda.

\begin{tabular}{|c|c||c|c|c|c|}
\hline Caso de estudio & \multicolumn{1}{|c||}{$\begin{array}{c}\text { Temperatura de } \\
\text { consigna }\end{array}$} & \multicolumn{4}{|c|}{$\begin{array}{c}\text { Demanda de energía acumulada (kW) } \\
\text { para clo \& met (enero) }\end{array}$} \\
\cline { 3 - 6 } & & 0.3 clo & 0.6 clo & 1.2 met & 1.6 met \\
\hline \hline Cafetería & $25^{\circ} \mathrm{C}$ & 14735.25 & 14840.86 & 15404.61 & 15920 \\
\hline
\end{tabular}

Tabla 16: Demanda acumulada de energía en el mes de enero, con valores variables de clo y met, para una temperatura de consigna de $25^{\circ} \mathrm{C}$. 


\section{Anexo 2: Código PMV y PPD}

El programa en BASIC que sigue, calcula los PMV y PPD para un conjunto de variables de entrada dados, basado en la normativa ISO 7730 y otros documentos de la serie dedicados a las condiciones térmicas.

// main.cpp

// PMVPPD

// Created by Roberto Robledo Fava on 18/05/18.

// Copyright (c) 2018 Roberto Robledo Fava. All rights reserved.

//

\# include $<$ stdio.h $>$

\# include "math.h"

\# include <stdlib.h>

int main()

\{

/*

CLO: Vestimenta

MET: Tasa metabólica

WME: Trabajo externo

TA: Temperatura del aire ${ }^{\circ} \mathrm{C}$

TR:Temperatura radiante media ${ }^{\circ} \mathrm{C}$

VEL: Velocidad relativa del aire $\mathrm{m} / \mathrm{s}$

RH: Humedad relativa \% 
PA:Presión parcial del vapor de agua Pa [PA No se incluye]

$* /$

float CLO,MET,WME,TA,TR,VEL,HR,PA, FNPS, ICL, M, W, MW, FCL, TAA, TRA, HCF, TCLA, P1, P2, P3, P4, P5, TCL, XF, XN, N, EPS, HCN, HC, HL1, HL2, HL3, HL4, HL5, HL6, TS, PMV, PPD;

$$
\begin{aligned}
& C L O=1.0 ; \\
& M E T=1.2 ; \\
& W M E=0 ; \\
& T A=19 ; \\
& T R=18 ; \\
& V E L=0.10 ; \\
& H R=40 ; \\
& P A=0 ;
\end{aligned}
$$$$
F N P S=\exp (16.6536-4030.183 /(T A+235)) ;
$$$$
P A=H R * 10 * F N P S \text {; // Presión de vapor saturado, }[k P a]
$$$$
I C L=0.155 * C L O ; / / \text { Aislamiento térmico de la ropa, }\left[m^{2} * K / W\right]
$$$$
M=M E T * 58.15 ; / / \text { Tasa metabólica, }\left[\mathrm{W} / \mathrm{m}^{2}\right]
$$$$
W=W M E * 58.15 ; / / \text { Trabajo externo, }\left[W / m^{2}\right] \text {. Valor alrededor de } 0
$$$$
M W=M-W ; \text { // Producción de Qi en el cuerpo humano }\left[W / m^{2}\right]
$$$$
\text { if }(I C L<=0.078)\{F C L=1+(1.29 * I C L) ;\} \text { else }\{F C L=1.05+(0.645 * I C L) ;\}
$$
// factor de superficie de la ropa

$H C F=12.1 * \operatorname{sqrt}(V E L) ;$ // Coefeciente de transferencia de energía por convección

$T A A=T A+273 ; / /$ Temp. aire en $[K]$

$T R A=T R+273 ; / /$ Temp. radiante media en $[K]$

\section{CÁlCULO DE LA TEMPERATURA DE SUPERFICIE DE ROPA POR ITERACIÓN}

$T C L A=T A A+(35.5-T A) /(3.5 * I C L+0.1) ; / /$ Primera iteración, que es una estimación. En las siguientes se encontrará el resultado correcto. 
$P 1=I C L * F C L ; / /$ Aislamiento térmico * F área ropa

$P 2=P 1 * 3.96$

$P 3=P 1 * 100$;

$P 4=P 1 * T A A ; / / P 1 *$ Taire $* /$

$P 5=(308.7-0.028 * M W)+(P 2 * \operatorname{pow}((T R A / 100), 4)) ; / /$-Fórmula modificada. Original ..(TRA/100)*4*/-

$X N=T C L A / 100 ; / / X N$ debe ser el "paso", 1/100 de la $1^{0}$ iteración

$X F=T C L A / 50$

$N=0 ;$ // Número de iteración (la actual)

$E P S=0.00015 ; / /$ Criterio de fin de iteración o bucle (valor final)

etiqueta1 : // GoTo STATEMENTS EES

$X F=(X F+X N) / 2$;

$H C N=2.38 * \operatorname{pow}(a b s(100 * X F-T A A), 0.25) ;$ // Coef. transf. calor por convección natural

if $(H C F>H C N)\{H C=H C F ;\}$ else $\{H C=H C N ;\}$

$X N=(P 5+P 4 * H C-P 2 * \operatorname{pow}(X F, 4)) /(100+(P 3 * H C)) ;$

$N=N+1$

if $(N>150)$ \{gotoetiqueta $2 ;\}$

if $(a b s(X N-X F)>E P S)\{$ gotoetiqueta $1 ;\}$

$T C L=100 * X N-273 ;$ // Temperatura superficial de la ropa

\section{CÁlCULO DE LAS COMPONENTES DE LA PÉRDIDA DE CALOR}

$H L 1=3.05 * 0.001 *(5733-(6.99 * M W)-P A) ;$ // Pérdida de calor por difusión a través de la piel

if $(M W>58.15)\{H L 2=0.42 *(M W-58.15)$; $\}$ else $\{H L 2=0 ;\}$ // Pérdida de calor por sudor

$H L 3=1.7 * 0.00001 * M *(5867-P A) ; \quad / /$ Pérdida de calor latente por respiración

$H L 4=0.0014 * M *(34-T A) ;$ // Pérdida de calor seco por la respiración 
$H L 5=3.96 * F C L *(\operatorname{pow}(X N, 4)-\operatorname{pow}((T R A / 100), 4)) ; / /$ Pérdida de calor por radiación

$H L 6=F C L * H C *(T C L-T A) ; / /$ Pérdida de calor por convección

\section{CÁLCULO DE LOS ÍNDICES PMV Y PPD}

$T S=0.303 * \exp (-0.036 * M)+0.028 ; / /$ Coeficiente de transferencia de sensación térmica

$P M V=T S *(M W-H L 1-H L 2-H L 3-H L 4-H L 5-H L 6) ; / /$ Voto medio estimado

$P P D=100.0-95.0 * \exp (-0.03353 *$ pow $(P M V, 4)-0.2179 *$ pow $(P M V, 2)) ;$ // Porcentaje de insatisfechos

printf( "PMV: \% f $\backslash n$ ", PMV);

printf( "PPD: \% f $\backslash n$ ", PPD);

etiqueta2 :

$P M V=99999 ;$

$P P D=10000$;

return 0 ;

\} 


\section{Anexo 3: Código PMV y PPD}

El programa en BASIC que sigue, calcula los PMV y PPD para un conjunto de variables de entrada dados, basado en la normativa ISO 7730 y otros documentos de la serie dedicados a las condiciones térmicas.

// PMVPPD

// Created by Roberto Robledo Fava on 18/05/18.

// Copyright (c) 2018 Roberto Robledo Fava. All rights reserved.

CLO: Vestimenta

MET: Tasa metabólica

WME: Trabajo externo

TA: Temperatura del aire ${ }^{\circ} \mathrm{C}$

TR:Temperatura radiante media ${ }^{\circ} \mathrm{C}$

VEL: Velocidad relativa del aire $\mathrm{m} / \mathrm{s}$

RH: Humedad relativa \%

PA:Presión parcial del vapor de agua Pa [PA No se incluye]

$I C L=0.155 * C L O ; / /$ Aislamiento térmico de la ropa, $\left[m^{2} * K / W\right]$

$M=M E T * 58.15 ; / /$ Tasa metabólica, $\left[\mathrm{W} / \mathrm{m}^{2}\right]$

$W=W M E * 58.15 ; / /$ Trabajo externo, $\left[\mathrm{W} / \mathrm{m}^{2}\right]$. Valor alrededor de 0

$M W=M-W ;$ // Producción de Qi en el cuerpo humano $\left[\mathrm{W} / \mathrm{m}^{2}\right]$

$N C L O=1.2$

$M=70 ;$ I/es la tasa metabolica, en vatios por metro cuadrado (W/m2)

$W M E=0$; //es la potencia mecanica efectiva, en vatios por metro cuadrado $(W / m 2)$; 
$T A=23.5$; //es la temperatura del aire, en grados Celsius $\left({ }^{\circ} \mathrm{C}\right)$;

$T c l=T A ;$ //es la temperatura de la superficie de la ropa, en grados Celsius $\left({ }^{\circ} \mathrm{C}\right)$.

$h r=3 ;$

$\operatorname{Tr}=25.5 ;$ //es la temperatura radiante media, en grados Celsius $\left({ }^{\circ} \mathrm{C}\right)$;

$V=0.1 ;$ //es la velocidad relativa del aire, en metros por segundo $(\mathrm{m} / \mathrm{s})$;

$R H=60 ; / /$ RH: Humedad relativa \%

Ar $A d u=0.77 ;$ // Ar /ADU es la fracción de superficie corporal participante en los intercambios de calor por radiación y depende de la postura del cuerpo. Puede tomarse el valor 0,77 para la mayoría de situaciones.

INICIALIZAMOS LAS VARIABLES $S=0$; factor $=500 ; R=0 ; C=0$; $f c l=0 ; h c=0 ;$ Balance $=1 ; E=0 ;$ Ediff $=0 ;$ Hres $=0$;

$W=W M E * 58.15 ; / /$ trabajo externo, en $W / m^{2}$

if $M<=58 ; M=58$; end

if $M>=232 ; M=232$; end

if $T A<=10 ; T A=10 ;$ end

if $W<=(0.0052 *(M-58)) ; W=0.0052 *(M-58)$; end

if $W>=1.2 ; W=1.2 ;$ end

if $V<=0.1 ; V=0.1$; end

if $V>=4 ; V=4$; end

Tsk $=35.7-0.0285 * M ; / /$ temperatura de la piel

$P A=(R H / 100) * 0.1333 * \exp (18.6686-4030.183 /(T A+235)) ; \quad / / e s$ la presión parcial del vapor de agua en el aire ambiente, en pascales (Pa)

$I c l=0.155 * N C L O ; / /$ aislamiento térmico de la vestimenta en $\left[\mathrm{m}^{2} \mathrm{~K} / \mathrm{W}\right]$

$I c l r=I c l ; / /$ resistencia térmica de la vestimenta

while abs (Balance) $>0.01$

$f c l=1.05+0.65 * I c l ; / /$ factor de superficie de la ropa

$E=0.42 *((M-W)-58) ; / /$ Pérdida de calor por sudor 
Ediff $=3.05 *(0.255 * T s k-3.36-P A) ; /$ pérdida de calor por difusión a través de la piel

$$
\text { Hres }=(1.73 e-2) * M *(5.867-P A)+(1.4 e-3) * M *(34-T A) ; / /
$$
pérdida de calor latente y seco por respiración

$T c l=T s k-I c l *(M-W-E-E d i f f-H r e s-S) ; / /$ temperatura superficial de la ropa

$$
h r=(5.67 e-8) * 0.95 * A r A d u *(\exp (4 * \log (273+T c l))-\exp (4 * \log (273+
$$
$\mathrm{Tr}))) /(\mathrm{Tcl}-\mathrm{Tr})$; //coeficiente de transferencia de calor por radiación, donde (5,67e-8) es la constante de Stefan Boltzman y (0.95) es la emisividad del atuendo

$h c=12.1 * \operatorname{sqr} t(V) ; / /$ Coeficiente de transferencia de calor por convección

$R=f c l * h r *(T c l-T r) ; / / T r a n s f e r e n c i a$ de calor por radiación desde la superficie de la ropa.

$C=f c l * h c *(T c l-T A) ; / /$ Transferencia convectiva de calor a través de la ropa.

Balance $=M-W-E-$ Ediff - Hres $-R-C-S$; //Balance térmico

if Balance $>0 S=S+$ factor $;$ factor $=$ factor $/ 2$; else $S=S-$ factor $;$ end end

$S=M-W-E-E d i f f-H r e s-R-C$; //es el calor acumulado por el organismo, cuyo valor permite conocer tiempos máximo de permanencia en un ambiente determinado.

$P M V=(0.303 * \exp (-0.036 * M)+0.028) * S ; / /$ Voto medio estimado $P P D=100-95 * \exp \left(-0.03353 *\left(P M V^{4}\right)-0.2179 *\left(P M V^{2}\right)\right) ; / / P o r c e n t a j e$ de insatisfechos 
